\title{
The quantum Hikita conjecture
}

\author{
Joel Kamnitzer \\ Department of Mathematics, University of Toronto, Toronto, Ontario, M5S 2E4
}

Michael McBreen

Department of Mathematics, University of Toronto, Toronto, Ontario, M5S 2E4

\author{
Nicholas Proudfoot \\ Department of Mathematics, University of Oregon, Eugene, OR 97403
}

\begin{abstract}
The Hikita conjecture relates the coordinate ring of a conical symplectic singularity to the cohomology ring of a symplectic resolution of the dual conical symplectic singularity. We formulate a quantum version of this conjecture, which relates the quantized coordinate ring of the first variety to the quantum cohomology of a symplectic resolution of the dual variety. We prove this conjecture for hypertoric varieties and for the Springer resolution.

Our paper includes an appendix, written by Ben Webster, which studies highest weights for quantizations of symplectic resolutions with isolated torus actions.
\end{abstract}

\section{Introduction}

A fascinating phenomenon in the theory of conical symplectic resolutions is that they tend to come in dual pairs. The exact definition of this notion of "symplectic duality" is somewhat in flux; a proposed definition in terms of a certain category $\mathcal{O}$ was formulated by the third author and collaborators in [BLPW16, Section 10], but it is not clear that this definition is flexible enough to encompass all of the examples that one wants to consider. Nonetheless, there is broad agreement on certain basic families of examples: the Springer resolution is dual to the Springer resolution for the Langlands dual group, [BGS96, Theorem 1.1.3], hypertoric varieties are dual to other hypertoric varieties [BLPW12, Theorem 1.2], affine type A quiver varieties are dual to other such varieties [Web17, Corollary 5.25]. Finite ADE quiver varieties are dual to slices in the affine Grassmannian for the Langlands dual group [BLPW16, Remark 10.7 and $\left[\mathrm{KTW}^{+} 19 \mathrm{~b}\right]$. Finally, and perhaps most important, given a linear representation of a reductive group, the Higgs branch of the associated 3-dimensional $N=4$ supersymmetric gauge theory (defined as a hyperkähler quotient) is dual to the Coulomb branch of the same theory (defined in [BFN18]), at least when the two spaces are sufficiently well behaved [Web].

Of the various manifestations of symplectic duality in terms of algebraic invariants of the resolutions, one of the most attractive is due to Hikita [Hik17]. Let $\tilde{X} \rightarrow X$ and $\tilde{X}^{!} \rightarrow X^{!}$ be a dual pair of conical symplectic resolutions, and let $T$ be a maximal torus in the Hamiltonian automorphism group of $\tilde{X}$. Hikita observed that, for many of the aforementioned 
examples, the coordinate ring of the fixed scheme $X^{T}$ is isomorphic to the cohomology ring of $\tilde{X}^{!}$. Specifically, he proved this for hypertoric varieties, finite type A quiver varieties, and the Hilbert scheme of points in the plane (which is self-dual), and he asked whether this phenomenon might hold for other examples of symplectic duality. For affine Grassmannian slices, this was proved by the first author and collaborators [KTW+19a, Theorem 8.1]. We will refer to this isomorphism of algebras as the Hikita conjecture.

The Hikita conjecture was extended by Nakajima [KTW ${ }^{+} 19 \mathrm{a}$, Conjecture 8.9], who proposed that the equivariant cohomology of $\tilde{X}^{!}$for the conical $\mathbb{G}_{m}$-action should coincide with the $B$-algebra of the quantized coordinate ring of $X$, with the equivariant parameter for the conical action identified with the quantization parameter for the coordinate ring. The $B$ algebra is an object that was introduced in [BLPW16, Section 5.2] to construct the standard and costandard objects of category $\mathcal{O}$ [BLPW16, Section 5.2]. We will refer to Nakajima's extension as the equivariant Hikita conjecture. In [ $\mathrm{KTW}^{+} 19 \mathrm{~b}$, Theorem 1.5], the first author and collaborators established a weak form of the equivariant Hikita conjecture for affine Grassmannian slices.

Our goal is to introduce yet another level of complexity to the Hikita conjecture. On one side of our conjecture, we will have the specialized quantum D-module of $\tilde{X}^{!}$. As a vector space, this is basically the equivariant quantum cohomology ring (see Remark 4.1), but it is equipped with the structure of a module over the Rees algebra of a certain ring of differential operators, where the module structure is related to quantum multiplication by divisors. The beautiful structures attached to the quantum D-module of a conical symplectic resolution have been the subject of much recent interest, starting with [OP10] and [BMO11]. For a sample of subsequent works, see [MO19, OS, MS13, MP15, AFO18, Oko15]. The word "specialized" refers to the fact that we identify the Rees parameter with the equivariant parameter for the conical action, which is a major simplification (see Remark 4.3).

On the other side of our conjecture, we have an object that serves as the universal source for graded traces of representations of the quantized coordinate ring of $X$, just as degree zero Hochschild homology serves as the universal source for ordinary traces. More specifically, let $\mathscr{A}$ be the canonical quantization of the universal filtered Poisson deformation of $\tilde{X}$. Thus $\mathscr{A}$ is a non-commutative algebra with a large centre, and the various central quotients of $\mathscr{A}$ are each quantizations of the coordinate ring of $X$. For example, if $X$ is the nilpotent cone in a reductive Lie algebra, then $\mathscr{A}$ is a finite extension of the corresponding universal enveloping algebra. The algebra $\mathscr{A}$ comes with two compatible gradings, one into weight spaces for the Hamiltonian torus action, and an additional $\mathbb{N}$-grading into weight spaces for the conical $\mathbb{G}_{m}$-action. Let $\mathscr{A}_{0}^{2}$ be the part of $\mathscr{A}$ that lies in weight 0 for the Hamiltonian torus action and degree 2 for the conical action. Let $S$ be the algebra with basis elements $q^{\lambda}$, where $\lambda$ is a element of the semigroup generated by certain weights of the Hamiltonian torus action 
called equivariant roots (Section 3.1). We then define $M$ to be the quotient of $S \otimes \operatorname{Sym} \mathscr{A}_{0}^{2}$ by the $S$-linear span of elements of the form $1 \otimes a b-q^{\lambda} \otimes b a$, where $a, b \in \mathscr{A}$ are elements of weight $\lambda$ and $-\lambda$, respectively. This vector space $M$ is not a ring, but rather a module under the action of the Rees algebra of a certain ring of differential operators (Proposition 3.5).

The definition of $M$ is motivated as follows. Let $V$ be a graded module over $\mathscr{A}$, and for any weight $\mu$ of the Hamiltonian torus, let $V_{\mu} \subset V$ be the corresponding weight space (see Section 3.6 for a more precise definition). If $V$ is suitably well behaved, then we have a graded trace map that takes an element $a \in \mathscr{A}_{0}$ to a power series where the coefficient of $q^{\mu}$ is equal to the trace of $a$ on $V_{\mu}$. We then prove that the graded trace map factors through $M$ (Proposition 3.15). For this reason, we call $M$ the D-module of graded traces. We note that after specializing $q=\hbar=1, M$ coincides with the degree zero Hochschild homology of $\mathscr{A}$ (Proposition 3.12), and we obtain the ordinary trace map for a finite dimensional representation of $\mathscr{A}$.

Our main conjecture (Conjecture 5.1) says that, after inverting some parameters associated with the equivariant roots, $M$ can be identified with the specialized quantum D-module of $\tilde{X}^{!}$, thus relating the quantization of $X$ to the quantum cohomology of $\tilde{X}^{!}$. We call this the quantum Hikita conjecture.

Conjecture 1.1. If $X$ and $X^{!}$are dual conical symplectic singularities and $\tilde{X}^{!}$is a symplectic resolution of $X^{!}$, then the D-module of graded traces for $X$ may be identified with the specialized quantum D-module of $\tilde{X}^{!}$away from the root hyperplanes.

Theorem 1.2. The quantum Hikita conjecture holds for hypertoric varieties and for Springer resolutions (Theorems 6.13 and 7.17 ).

In addition to being interesting in its own right, the quantum Hikita conjecture relates to various previous conjectures by specializing $q$. If we set $q$ equal to zero, then $M$ turns into $B$-algebra of $\mathscr{A}$ (Proposition 3.8 and Remark 3.9), and our conjecture specializes to a version of the equivariant Hikita conjecture (Remark 5.3). On the other hand, setting $q$ equal to $1, M$ turns into the degree zero Hochschild homology of $\mathscr{A}$, which is conjecturally related to the intersection cohomology of $X^{!}$[Pro14, Conjecture 3.6]. Similarly, the quantum cohomology of $\tilde{X}^{!}$at $q=1$ is also conjecturally related to the intersection cohomology of $X^{!}$ [MP15, Conjecture 2.5]. Thus our conjecture provides a bridge between these two previous conjectures of the second and third authors (Remark 5.7).

Remark 1.3. One of the original motivations for this work was the case where $X$ (respectively $X^{!}$) is the Coulomb (respectively Higgs) branch of a 3-dimensional $N=4$ supersymmetric gauge theory. In this case, there is a clear heuristic for the relation between the 
module of graded traces for $X$ and the specialized quantum D-module of $\tilde{X}^{!}$. Indeed, in this case the specialized quantum D-module is encoded in the differential relations satisfied by a certain function called the $I$-function. The $I$-function is a generating function for equivariant volumes of moduli spaces of quasimaps from a rational curve into $\tilde{X}^{!}$. These quasimaps, in turn, are closely related to the moduli spaces used to define $X$ in [BFN18]. In a forthcoming paper, the first author together with Justin Hilburn and Alex Weekes will prove that the quantization of $X$ acts on the homology of certain quasimap moduli spaces for $\tilde{X}^{!}$.

Remark 1.4. A different possible line of investigation is to replace the equivariant cohomology of $\tilde{X}^{!}$by its equivariant $K$-theory. Then the specialized quantum D-module must be replaced by a module over difference operators, which has in many respects proved to be an even richer object [OS, AFO18, Oko15. It would be interesting to see how our conjecture adapts to this setting.

Acknowledgments: The authors would like to thank Alexander Braverman for proposing the problem of formulating a quantum version of the Hikita conjecture and for many helpful discussions. The authors are also grateful to Roman Bezrukavnikov, Pavel Etingof, Davide Gaiotto, Sam Gunningham, Ivan Losev, Davesh Maulik, Hiraku Nakajima, Andrei Negut, Andrei Okounkov, Peng Shan and Ben Webster for stimulating conversations. JK was supported by an NSERC discovery grant. MM completed part of this work at the Massachusetts Institute of Technology, the Hausdorff Center for Mathematics, and the Yau Mathematical Sciences Center. NP was supported by NSF grant DMS-1565036 and would like to thank le Château de Trintange for its hospitality during the last stages of the completion of this manuscript.

\section{Conical symplectic singularities}

Let $X$ be a conical symplectic singularity of weight two. By this we mean that $X$ is a normal affine Poisson variety over $\mathbb{C}$ equipped with an action of $\mathbb{G}_{m}$ satisfying the following conditions:

- the coordinate ring $\mathcal{O}(X)$ is non-negatively graded by the action of $\mathbb{G}_{m}$, with the degree zero part consisting only of constant functions and the degree one part being zerd 1

- the Poisson bracket has degree -2 with respect to this grading

- the Poisson bracket is induced by a symplectic form $\omega^{\text {reg }}$ on the smooth locus $X^{\text {reg }}$

\footnotetext{
${ }^{1}$ This last condition rules out the degenerate example $X=\mathbb{C}^{2}$, or anything with a factor of $\mathbb{C}^{2}$.
} 
- for some (equivalently any) projective resolution $\pi: \tilde{X} \rightarrow X$, the 2 -form $\pi^{*} \omega^{\text {reg }}$ extends to a (possibly degenerate) 2-form on $\tilde{X}$.

Examples include the nilpotent cone of a simple Lie algebra, hypertoric varieties, quiver varieties, and certain subvarieties of the affine Grassmannian.

\subsection{The Hamiltonian automorphism group}

Let $\mathcal{O}(X)^{2}$ be the degree 2 part of $\mathcal{O}(X)$. Since the Poisson bracket on $\mathcal{O}(X)$ has degree $-2, \mathcal{O}(X)^{2}$ is a Lie subalgebra of $\mathcal{O}(X)$. This Lie algebra acts by graded endomorphisms on $\mathcal{O}(X)$. Assume that there exists a reductive group $\operatorname{Aut}(X)$, whose Lie algebra is $\mathcal{O}(X)^{2}$, and which acts faithfully by Poisson automorphisms on $\mathcal{O}(X)$, integrating the action of $\mathcal{O}(X)^{2}$.

Remark 2.1. If $X$ admits a symplectic resolution $\tilde{X}$, then the Lie algebra $\mathcal{O}(X)^{2}$ may be identified with the Lie algebra of Hamiltonian vector fields on $\tilde{X}$. For this reason, we refer to $\operatorname{Aut}(X)$ as the Hamiltonian automorphism group of $X$. If, in addition, $\tilde{X}$ admits a hyperkähler metric compatible with the symplectic form, then we expect $\operatorname{Aut}(X)$ to be the complexification of the group of hyperkähler automorphisms of $\tilde{X}$. This gives at least a heuristic reason to believe that the Lie algebra $\mathcal{O}(X)^{2}$ integrates to a reductive group.

Let $T \subset \operatorname{Aut}(X)$ be a maximal torus, and let $\mathfrak{t}:=\operatorname{Lie}(T)$ be the Lie algebra of $T$. The action of $T$ on $X$ induces a second grading on coordinate ring $\mathcal{O}(X)$ by the group $\mathfrak{t}_{\mathbb{Z}}^{*}:=\operatorname{Hom}\left(T, \mathbb{G}_{m}\right)$. Since the action of $T$ commutes with the action of $\mathbb{G}_{m}$, this second grading is compatible with the grading by $\mathbb{N}$. For any $\lambda \in \mathfrak{t}_{\mathbb{Z}}^{*}$ and $k \in \mathbb{N}$, we let define $\mathcal{O}(X)_{\lambda}$, $\mathcal{O}(X)^{k}$, and $\mathcal{O}(X)_{\lambda}^{k}:=\mathcal{O}(X)_{\lambda} \cap \mathcal{O}(X)^{k}$ to be the corresponding isotypic components for the actions of $T, \mathbb{G}_{m}$, and $T \times \mathbb{G}_{m}$, respectively. Since $\operatorname{Aut}(X)$ is reductive, the zero root space $\mathcal{O}(X)_{0}^{2} \subset \mathcal{O}(X)^{2}$ is equal to the Cartan subalgebra $\mathfrak{t} \subset \mathcal{O}(X)^{2}$.

\subsection{Deformation and quantization}

Choose a $\mathbb{Q}$-factorial terminalization $\tilde{X}$ of $X$, as in [Los, Proposition 2.3], and consider the smooth locus $\tilde{X}^{\text {sm }} \subset \tilde{X}$. Let $\tilde{\mathscr{X}}$ be the universal filtered Poisson deformation of $\tilde{X}$, which has base $H^{2}\left(\tilde{X}^{\text {sm }} ; \mathbb{C}\right)$. Let $\mathscr{X}:=\operatorname{Spec} \mathcal{O}(\tilde{\mathscr{X}})$, which is a deformation of $X$ over $H^{2}\left(\tilde{X}^{\mathrm{sm}} ; \mathbb{C}\right)$. Two different choices of $\tilde{X}$ will yield two isomorphic families $\mathscr{X}$, and the isomorphism between them is canonical up to the action of the Namikawa Weyl group [Los, Corollary 2.13].

Let $\mathscr{A}$ be the canonical quantization of $\mathscr{X}$. This is an $\mathbb{N}$-graded algebra over the ring Sym $H_{2}\left(\tilde{X}^{\mathrm{sm}} ; \mathbb{C}\right) \otimes \mathbb{C}[\hbar]$, with $H_{2}\left(\tilde{X}^{\mathrm{sm}} ; \mathbb{C}\right)$ and $\hbar$ both in degree 2 . If we set $\hbar$ equal to 1 , we obtain the canonical filtered quantization of [Los, Proposition 3.3]. The existence of such a quantization follows from the work of Bezrukavnikov-Kaledin and Losev; see [BPW16, Sections 3.1-3.3] for details. 
Let $\mathscr{A}^{2}$ denote the degree 2 part of $\mathscr{A}$. This is naturally a Lie algebra, with Lie bracket given by $\hbar^{-1}$ times the commutator. The centre of the Lie algebra $\mathscr{A}^{2}$ contains $H_{2}\left(\tilde{X}^{\mathrm{sm}} ; \mathbb{C}\right) \oplus$ $\mathbb{C} \hbar$, and the quotient of $\mathscr{A}^{2}$ by this subalgebra is canonically isomorphic to $\mathcal{O}(X)^{2}$. That is, we have an exact sequence of Lie algebras

$$
0 \rightarrow H_{2}\left(\tilde{X}^{\mathrm{sm}} ; \mathbb{C}\right) \oplus \mathbb{C} \hbar \rightarrow \mathscr{A}^{2} \rightarrow \mathcal{O}(X)^{2} \rightarrow 0
$$

where $H_{2}\left(\tilde{X}^{\mathrm{sm}} ; \mathbb{C}\right) \oplus \mathbb{C} \hbar$ is endowed with the trivial Lie bracket. For any $x \in \mathscr{A}^{2}$, let $\bar{x}$ denote the image of $x$ in $\mathcal{O}(X)^{2}$.

The Lie algebra $\mathscr{A}^{2}$ acts on $\mathscr{A}$ by $\hbar^{-1}$ times the commutator; furthermore, the central subalgebra $H_{2}\left(\tilde{X}^{\mathrm{sm}} ; \mathbb{C}\right) \oplus \mathbb{C} \hbar$ acts trivially, so we obtain an action of the Lie algebra $\mathcal{O}(X)^{2}$ on $\mathscr{A}$. Since the action of $\mathcal{O}(X)^{2}$ on $\mathcal{O}(\mathscr{X})$ integrates to an action of $\operatorname{Aut}(X)$ and $\mathscr{A}$ is a flat deformation of $\mathcal{O}(\mathscr{X})$ over the affine line, the action of $\mathcal{O}(X)^{2}$ on $\mathscr{A}$ also integrates to an action on $\operatorname{Aut}(X)$. This endows $\mathscr{A}$ with a direct sum decomposition

$$
\mathscr{A}=\bigoplus_{\lambda \in \mathfrak{t}_{\mathbb{Z}}^{*}} \mathscr{A}_{\lambda}
$$

into $T$-weight spaces, where

$$
\mathscr{A}_{\lambda}:=\left\{a \in \mathscr{A} \mid[x, a]=\hbar\langle\lambda, \bar{x}\rangle a \text { for all } x \in \mathscr{A}_{0}^{2}\right\} .
$$

This decomposition is compatible with the grading by $\mathbb{N}$. Taking zero weight spaces in the exact sequence (1), we obtain an exact sequence

$$
0 \rightarrow H_{2}\left(\tilde{X}^{\mathrm{sm}} ; \mathbb{C}\right) \oplus \mathbb{C} \hbar \rightarrow \mathscr{A}_{0}^{2} \rightarrow \mathfrak{t} \rightarrow 0
$$

which we call the quantization exact sequence. This exact sequence will play a major role in the formulation of our main conjecture.

Remark 2.2. Since $\mathscr{A}_{0}^{2}$ is abelian, the quantization exact sequence splits. Choosing a splitting is equivalent to choosing a quantum comoment map for the action of $T$ on $\mathscr{A}$.

There are two main examples which we will work with in this paper: hypertoric varieties and the Springer resolution.

Example 2.3. Suppose that $X$ is the affine hypertoric variety obtained as a symplectic quotient of $T^{*} \mathbb{C}^{n}$ by a subtorus $K \subset \mathbb{G}_{m}^{n}$. Then we may take $T=\mathbb{G}_{m}^{n} / K$, and $\mathscr{A}$ is isomorphic to the hypertoric enveloping algebra (Section 6.2). If $y_{i}$ is the $i^{\text {th }}$ coordinate function on $\mathbb{C}^{n}$, then $\mathscr{A}_{0}^{2}$ has basis $\left\{\hbar, y_{1} \partial_{1}, \ldots, y_{n} \partial_{n}\right\}$. The map from $\mathscr{A}_{0}^{2} / \mathbb{C} \hbar \cong \operatorname{Lie}\left(\mathbb{G}_{m}^{n}\right)$ to 
$\mathfrak{t}$ is induced by the map of algebraic groups from $\mathbb{G}_{m}^{n}$ to $T$. This example will be studied in greater detail in Section 6 .

Example 2.4. Let $G$ be a semisimple complex group and let $X \subset \mathfrak{g}^{*}$ be the union of those coadjoint orbits that are preserved by dilations. If we use the Killing form to identify $\mathfrak{g}^{*}$ with $\mathfrak{g}$, then $X$ is taken to the nilpotent cone of $\mathfrak{g}$, so we will refer to $X$ as the nilpotent cone. Then $X$ is a conical symplectic singularity of weight two, where the Poisson structure comes from restricting the usual Poisson structure on $\mathfrak{g}^{*}$ and the action of $\mathbb{G}_{m}$ is by the inverse square of scalar multiplication. Furthermore, $X$ admits a symplectic resolution $\tilde{X}=$ $T^{*}(G / B)$, known as the Springer resolution. The group $\operatorname{Aut}(X)$ is isomorphic to the quotient of $G$ by its centre, and $T$ is the quotient of the maximal torus of $G$ by the centre of $G$. The group $H^{2}(\tilde{X} ; \mathbb{C})$ is canonically isomorphic to $\mathfrak{t}^{*}$. The universal Poisson deformation $\tilde{\mathscr{X}}$ is isomorphic to the Grothedieck-Springer resolution $\tilde{\mathfrak{g}}^{*}$, and its affinization $\mathscr{X}$ is isomorphic to $\mathfrak{g}^{*} \times_{\mathfrak{t}^{*} / W} \mathfrak{t}^{*}$. The canonical filtered quantization is identified with the enhanced enveloping algebra $\mathcal{A}:=U \mathfrak{g} \otimes_{Z(U \mathfrak{g})}$ Sym $\mathfrak{t}$, and $\mathscr{A}$ is the Rees algebra with respect to the PBW filtration. The space $\mathscr{A}_{0}^{2}$ is generated by $\hbar$ and vectors of the form $x_{1} \otimes 1+1 \otimes x_{2}$ for $x_{1}, x_{2} \in \mathfrak{t}$. The map from $\mathscr{A}_{0}^{2}$ to $\mathfrak{t}$ in the quantization exact sequence takes $\hbar$ to 0 and $x_{1} \otimes 1+1 \otimes x_{2}$ to $x_{1}$.

\section{Algebraic construction}

In this section we fix a conical symplectic singularity $X$ and a $\mathbb{Q}$-factorial terminalization $\tilde{X}$, and we use the canonical quantization $\mathscr{A}$ from Section 2.2 to define the D-module of graded traces.

\subsection{Equivariant roots}

Let $\mathscr{A}^{+} \subset \mathscr{A}$ be the two-sided ideal spanned by classes of positive degree with respect to the $\mathbb{N}$-grading, and consider the $T$-vector space $\mathscr{A}^{+} /\left(\mathscr{A}^{+} \cdot \mathscr{A}^{+}\right)$. Let $\Sigma \subset \mathfrak{t}_{\mathbb{Z}}^{*}$ be the set of nonzero weights of $\mathscr{A}^{+} /\left(\mathscr{A}^{+} \cdot \mathscr{A}^{+}\right)$. Motivated by [Oko15, Definition 3.1], we will refer to $\Sigma$ as the set of equivariant roots of $X$.

Remark 3.1. Setting $\hbar$ equal to zero gives a canonical surjective map of $T$-representations from $\mathscr{A}^{+} /\left(\mathscr{A}^{+} \cdot \mathscr{A}^{+}\right)$to the Zariski cotangent space to $\mathscr{X}$ at the unique $\left(T \times \mathbb{G}_{m}\right)$-fixed point; we expect this map to be an isomorphism. This in turn maps to the Zariski cotangent space to $X$, inducing a bijection on nonzero weights. Okounkov defines the equivariant roots by choosing a symplectic resolution (if it exists) and taking the union of the nonzero weights in the cotangent spaces of all of the $T$-fixed points of the resolution 2 We expect that our

\footnotetext{
${ }^{2}$ Okounkov uses tangent spaces rather than cotangent spaces, but the weights are the same, since the action of $T$ preserves the symplectic form.
} 
definition will coincide with Okounkov's when a symplectic resolution exists.

Fix a cocharacter $\xi \in \mathfrak{t}_{\mathbb{Z}}$ such that $\langle\lambda, \xi\rangle \neq 0$ for all $\lambda \in \Sigma$, and let

$$
\Sigma_{+}:=\{\lambda \in \Sigma \mid\langle\lambda, \xi\rangle>0\}
$$

We will call elements of $\Sigma_{+}$positive equivariant roots. Let

$$
\mathscr{A}_{+}:=\bigoplus_{\langle\lambda, \xi\rangle>0} \mathscr{A}_{\lambda} \quad \text { and } \quad \mathscr{A}_{-}:=\bigoplus_{\langle\lambda, \xi\rangle<0} \mathscr{A}_{\lambda}
$$

The following lemma says that the right ideal of $\mathscr{A}$ generated by $\mathscr{A}_{+}$is in fact generated by the elements of $\mathscr{A}_{\lambda}$ for $\lambda \in \Sigma_{+}$.

Lemma 3.2. If $a \in \mathscr{A}_{+}^{m}$, then there exist positive equivariant roots $\lambda_{1}, \ldots, \lambda_{n}$ (possibly not distinct), along with elements $y_{i} \in \mathscr{A}_{\lambda_{i}}$ and $z_{i} \in \mathscr{A}$ for all $i$, such that

$$
a=\sum_{i=1}^{n} y_{i} z_{i} .
$$

Proof. We proceed by induction on $m$. We may assume that $a \in \mathscr{A}_{\mu}^{m}$ for some $\mu \in \mathfrak{t}_{\mathbb{Z}}^{*}$ with $\langle\mu, \xi\rangle>0$, and we may assume that the statement holds for all elements of $\mathscr{A}_{+}^{k}$ when $k<m$.

If $a \notin \mathscr{A}^{+} \cdot \mathscr{A}^{+}$, then a represents a nonzero element of $\mathscr{A}^{+} /\left(\mathscr{A}^{+} \cdot \mathscr{A}^{+}\right)$, in which case $\mu \in \Sigma_{+}$and we are done. Thus we may assume that $a \in \mathscr{A}^{+} \cdot \mathscr{A}^{+}$. This means that we can write $a=\sum_{j} b_{j} c_{j}$, where $b_{j} \in \mathscr{A}_{\mu_{j}}^{p_{j}}$ and $c_{j} \in \mathscr{A}_{\mu-\mu_{j}}^{m-p_{j}}$ for some elements $\mu_{j} \in \mathfrak{t}_{\mathbb{Z}}^{*}$ and $p_{j} \in \mathbb{N}$ with $0<p_{j}<m$ for all $j$. If $\left\langle\mu_{j}, \xi\right\rangle>0$, then we may apply our inductive hypothesis to $b_{j}$, and thus write $b_{j} c_{j}$ in the desired form. Alternatively, if $\left\langle\mu_{j}, \xi\right\rangle \leq 0$, then $\left\langle\mu-\mu_{j}, \xi\right\rangle \geq\langle\mu, \xi\rangle>0$, so we may apply our inductive hypothesis to $c_{j}$. Finally, we note that

$$
b_{j} c_{j}=c_{j} b_{j}+\left[b_{j}, c_{j}\right]=c_{j} b_{j}+\hbar d_{j}
$$

for some $d_{j} \in \mathscr{A}_{\mu}^{m-2}$. Applying our inductive hypothesis to both $c_{j}$ and $d_{j}$, we may again write $b_{j} c_{j}$ in the desired form.

\subsection{The ring $R$}

Let

$$
S:=\mathbb{C}\left\{q^{\lambda} \mid \lambda \in \mathbb{N} \Sigma_{+}\right\} \subset \mathbb{C}\left\{q^{\lambda} \mid \lambda \in \mathfrak{t}_{\mathbb{Z}}^{*}\right\} \cong \mathcal{O}(T) .
$$

The fact that $T$ acts effectively on $X$ implies that $\Sigma_{+}$spans $\mathfrak{t}_{\mathbb{Z}}^{*}$, and therefore that Spec $S$ is a (possibly non-normal) affine $T$-toric variety with a unique fixed point $0 \in \operatorname{Spec} S$. 
Let

$$
R:=S \otimes \operatorname{Sym} \mathscr{A}_{0}^{2}
$$

which we endow with a $\mathbb{C}[\hbar]$-algebra structure by setting

$$
x q^{\lambda}=q^{\lambda}(x+\hbar\langle\lambda, \bar{x}\rangle)
$$

for all $\lambda \in \mathbb{N} \Sigma_{+}$and $x \in \mathscr{A}_{0}^{2}$. The ring $R$ is $\mathbb{N}$-graded, with $S$ in degree zero.

Remark 3.3. For any $c \in H^{2}\left(\tilde{X}^{\mathrm{sm}} ; \mathbb{C}\right)$, let $R^{c}$ be the quotient of $R$ by the ideal generated by $\theta-\hbar\langle\theta, c\rangle$ for all $\theta \in H_{2}\left(\tilde{X}^{\mathrm{sm}} ; \mathbb{C}\right) \subset \mathscr{A}_{0}^{2}$, and let $R_{T}^{c}$ be the ring obtained from $R^{c}$ by localizing from Spec $S$ to $T$. Then $R_{T}^{c}$ is (non-canonically) isomorphic to the Rees algebra of differential operators on $T$, filtered by order. If we choose a splitting of the quantization exact sequence (2), then we obtain a ring isomorphism by sending an element of $\mathfrak{t}$ to $\hbar$ times the corresponding translation invariant vector field on $T$. For this reason, we think of $R$ as a ring of differential operators with values in $H_{2}\left(\tilde{X}^{\mathrm{sm}} ; \mathbb{C}\right)$.

In the sections that follow, we will be particularly interested in the localization

$$
S_{\mathrm{reg}}:=S\left[\frac{1}{1-q^{\lambda}} \mid \lambda \in \Sigma_{+}\right]
$$

We will also need to invert the same collection of elements in $R$; this requires care since $R$ is non-commutative. Let $\mathfrak{S} \subset R$ be the multiplicative subset generated by $\left(1-q^{\lambda}\right), \lambda \in \Sigma_{+}$.

Lemma 3.4. The set $\mathfrak{S}$ satisfies the Ore condition. That is, for any $s \in \mathfrak{S}$ and $r \in R$, there exists $s^{\prime} \in \mathfrak{S}$ and $r^{\prime} \in R$ such that $s^{\prime} r=r^{\prime} s$.

Proof. For any homogeneous $r \in R$ let $\operatorname{deg}(r) \in \mathbb{N}$ be its degree. First, we claim that, for any $N \geq \operatorname{deg}(r)$, the commutator $\left[r,\left(1-q^{\lambda}\right)^{N}\right]$ is right divisible by $\left(1-q^{\lambda}\right)^{N-\operatorname{deg}(r) / 2}$. We prove this claim by induction on the degree of $r$. When $\operatorname{deg}(r)=2$, we can immediately reduce to the case where $r=x \in \mathscr{A}_{0}^{2}$, and we have

$$
\left[x,\left(1-q^{\lambda}\right)^{N}\right]=-N \hbar\langle\lambda, \bar{x}\rangle q^{\lambda}\left(1-q^{\lambda}\right)^{N-1} .
$$

Suppose our claim holds for all homogeneous elements of $R$ of degree less than $n=\operatorname{deg}(r)$. We may reduce to the case where $r=r^{\prime} x$ for some $x \in \mathscr{A}_{0}^{2}$ and $r^{\prime} \in R$ with $\operatorname{deg}\left(r^{\prime}\right)=n-2$. Then

$$
\begin{aligned}
{\left[r,\left(1-q^{\lambda}\right)^{N}\right] } & =r^{\prime} x\left(1-q^{\lambda}\right)^{N}-\left(1-q^{\lambda}\right)^{N} r^{\prime} x \\
& =r^{\prime} x\left(1-q^{\lambda}\right)^{N}-r^{\prime}\left(1-q^{\lambda}\right)^{N} x+\left[r^{\prime},\left(1-q^{\lambda}\right)^{N}\right] x
\end{aligned}
$$


Our inductive hypothesis tells us that there is some $r^{\prime \prime} \in R$ such that

$$
\left[r^{\prime},\left(1-q^{\lambda}\right)^{N}\right]=r^{\prime \prime}\left(1-q^{\lambda}\right)^{N-n / 2+1}
$$

thus

$$
\left[r,\left(1-q^{\lambda}\right)^{N}\right]=r^{\prime} x\left(1-q^{\lambda}\right)^{N}-r^{\prime}\left(1-q^{\lambda}\right)^{N} x+r^{\prime \prime}\left(1-q^{\lambda}\right)^{N-n / 2+1} x .
$$

The claim then follows from two applications of Equation (3).

We return to our lemma. We may assume that $r$ is homogeneous. Write

$$
s:=\prod_{\lambda \in \Sigma_{+}}\left(1-q^{\lambda}\right)^{N_{\lambda}}
$$

and let

$$
s^{\prime}:=\prod_{\lambda \in \Sigma_{+}}\left(1-q^{\lambda}\right)^{M},
$$

where $M:=\operatorname{deg}(r) / 2+\max \left\{N_{\lambda} \mid \lambda \in \Sigma_{+}\right\}$. We have $s^{\prime} r=r s^{\prime}-\left[r, s^{\prime}\right]$, and our claim implies that $\left[r, s^{\prime}\right]$ is right divisible by $\prod_{\lambda \in \Sigma_{+}}\left(1-q^{\lambda}\right)^{N_{\lambda}}=s$. This concludes the proof.

We can thus define the Ore localization $R_{\text {reg }}:=\mathfrak{S}^{-1} R$, which is isomorphic as a graded vector space to $S_{\text {reg }} \otimes \operatorname{Sym} \mathscr{A}_{0}^{2}$.

\subsection{The module $M$}

We endow $S \otimes \mathscr{A}_{0}$ with the structure of an $\mathbb{N}$-graded left $R$-module by putting

$$
x \cdot\left(q^{\lambda} \otimes a\right):=q^{\lambda} \otimes(x+\hbar\langle\lambda, \bar{x}\rangle) a \quad \text { and } \quad q^{\mu} \cdot\left(q^{\lambda} \otimes a\right):=q^{\lambda+\mu} \otimes a
$$

for all $x \in \mathscr{A}_{0}^{2}, a \in \mathscr{A}_{0}$, and $\lambda, \mu \in \mathbb{N} \Sigma_{+}$. Let

$$
J:=\sum_{\lambda \in \mathbb{N} \Sigma_{+}} S \cdot\left\{1 \otimes a b-q^{\lambda} \otimes b a \mid a \in \mathscr{A}_{\lambda}, b \in \mathscr{A}_{-\lambda}\right\} \subset S \otimes \mathscr{A}_{0}
$$

A priori, $J$ is a graded $S$-submodule of $S \otimes \mathscr{A}_{0}$. Proposition 3.5 says that it is in fact an $R$-submodule.

Proposition 3.5. $J$ is an $R$-submodule of $S \otimes \mathscr{A}_{0}$.

Proof. It is sufficient to check that, if $x \in \mathscr{A}_{0}^{2}, \lambda \in \mathbb{N} \Sigma_{+}, a \in \mathscr{A}_{\lambda}$, and $b \in \mathscr{A}_{-\lambda}$, then we 
have $x \cdot\left(1 \otimes a b-q^{\lambda} \otimes b a\right) \in J$. Indeed, we have

$$
\begin{aligned}
x \cdot\left(1 \otimes a b-q^{\lambda} \otimes b a\right) & =1 \otimes x a b-q^{\lambda} \otimes(x+\hbar\langle\lambda, \bar{x}\rangle) b a \\
& =1 \otimes x a b-q^{\lambda} \otimes x b a+q^{\lambda} \otimes[x, b] a \\
& =1 \otimes x a b-q^{\lambda} \otimes b x a,
\end{aligned}
$$

which is an element of $J$.

Our main object of study will be the graded $R$-module

$$
M:=\left(S \otimes \mathscr{A}_{0}\right) / J
$$

which we call the D-module of graded traces (see Section 3.6 for the motivation behind this terminology). We will be particularly interested in the localization

$$
M_{\text {reg }}:=R_{\text {reg }} \otimes_{R} M
$$

Example 3.6. We illustrate these constructions when $X$ is the Kleinian singularity of type $A_{1}$, or (equivalently) the nilpotent cone in $\mathfrak{s l}_{2}^{*}$. Choose a basis $\left\{\hbar, a_{1}, a_{2}\right\}$ for $\mathscr{A}_{0}^{2}$ such that $\Sigma_{+} \subset \mathfrak{t}^{*}$ consists of a single element that evaluates to 1 on both $\bar{a}_{1}$ and $\bar{a}_{2}$. Then $S=\mathbb{C}[q]$ and $R$ is generated over $\mathbb{C}[\hbar]$ by $q, a_{1}$, and $a_{2}$. The classes $a_{1}$ and $a_{2}$ commute with each other, and $a_{i} q=q\left(a_{i}+\hbar\right)$. The $\mathbb{C}[\hbar]$-algebra $\mathscr{A}$ has generators $r_{+}, r_{-}, a_{1}, a_{2}$ and relations

$$
\begin{gathered}
r_{+} r_{-}=a_{1} a_{2}, \quad r_{-} r_{+}=\left(a_{1}+\hbar\right)\left(a_{2}+\hbar\right) \\
{\left[a_{i}, r_{+}\right]=\hbar r_{+}, \quad\left[a_{i}, r_{-}\right]=-\hbar r_{-}, \quad\left[a_{1}, a_{2}\right]=0 .}
\end{gathered}
$$

This algebra is an example of a hypertoric enveloping algebra (Section 6.2), and it is also the Rees algebra of the enhanced enveloping algebra of $\mathfrak{s l}_{2}$. To see this, we identify $r_{+}$with $E,-r_{-}$with $F, a_{1}+a_{2}+\hbar$ with $H$, and $a_{1}-a_{2}$ with the square root of the central element $C=2 E F+2 F E+H^{2}+\hbar^{2}$.

Now let us compute the module $M$. We have $\mathscr{A}_{0}=\mathbb{C}\left[\hbar, a_{1}, a_{2}\right]$, which implies that $S \otimes \mathscr{A}_{0}$ is a free $R$-module of rank 1 . So it remains to compute the left ideal $J \subset S \otimes \mathscr{A}_{0} \cong R$. By the definition, $J$ contains the element

$$
r_{+} r_{-}-q r_{-} r_{+}=a_{1} a_{2}-q\left(a_{1}+\hbar\right)\left(a_{2}+\hbar\right)=a_{1} a_{2}(1-q) .
$$

It is a special case of Proposition 6.8 that $J$ is in fact generated by this single element. 


\subsection{Specializations and Hochschild homology}

We will now relate some specializations of $M$ to the degree zero Hochschild homology of $\mathscr{A}$. Recall that if $A$ is any ring and $B$ is an $(A, A)$-bimodule, then

$$
H H_{0}(A, B):=B / \mathbb{C}\{a b-b a: a \in A, b \in B\} .
$$

We will write $H H_{0}(A):=H H_{0}(A, A)$ where $A$ is the regular bimodule.

We begin by considering the specialization

$$
M_{0}:=\mathbb{C}_{0} \otimes_{S} M,
$$

where the map $S \rightarrow \mathbb{C}_{0}$ is given by setting $q$ equal to zero (or, equivalently, by evaluation at the unique $T$-fixed point of the toric variety $\operatorname{Spec} S$ ). Note that $M_{0}$ carries the structure of a Sym $\mathscr{A}_{0}^{2}$ module since $\mathscr{A}_{0}^{2}$ lies in the centre of $\mathscr{A}_{0}$. This specialization is closely related to the algebra

$$
B(\mathscr{A}):=\mathscr{A}_{0} / \sum_{\langle\mu, \xi\rangle>0}\left\{a b \mid a \in \mathscr{A}_{\mu}, b \in \mathscr{A}_{-\mu}\right\},
$$

which was introduced in [BLPW16, Section 5.1] to study the representation theory $\mathscr{A}$, and which also appears in the equivariant Hikita conjecture [KTW ${ }^{+}$19a, Conjecture 8.9].

Remark 3.7. The definition of $B(\mathscr{A})$ in [BLPW16, Section 5.1] uses the inequality $\langle\mu, \xi\rangle<0$ rather than $\langle\mu, \xi\rangle>0$. This means that the algebra that we study in this paper is related to category $\mathcal{O}$ for the parameter $-\xi$ rather than $\xi$.

Proposition 3.8. We have an isomorphism $M_{0} \cong H H_{0}(B(\mathscr{A}))$ of Sym $\mathscr{A}_{0}^{2}$-modules.

Proof. Let

$$
\tilde{J}:=\sum_{0 \neq \lambda \in \mathbb{N} \Sigma_{+}} S \cdot\left\{1 \otimes a b-q^{\lambda} \otimes b a \mid a \in \mathscr{A}_{\lambda}, b \in \mathscr{A}_{-\lambda}\right\} \subset S \otimes \mathscr{A}_{0} .
$$

Thus $\tilde{J}$ is defined in the same way as $J$, except that we do not allow $\lambda=0$. Let $\tilde{M}:=R / \tilde{J}$ and $\tilde{M}_{0}:=\mathbb{C}_{0} \otimes_{S} \tilde{M}$.

We claim that $\tilde{M}_{0}$ is isomorphic to $B(\mathscr{A})$. Indeed, $\tilde{M}_{0}$ is the quotient of $\mathscr{A}_{0}$ by the ideal $\tilde{J}_{0}:=\mathbb{C}_{0} \otimes_{S} \tilde{J}$, and it is clear that $\tilde{J}_{0}$ is contained in the kernel of the surjection from $\mathscr{A}_{0}$ to $B(\mathscr{A})$. We need to show that, if $\langle\mu, \xi\rangle>0, a \in \mathscr{A}_{\mu}$, and $b \in \mathscr{A}_{-\mu}$, then $a b \in \tilde{J}_{0}$. To see this, write

$$
a=\sum y_{i} z_{i}
$$

as in Lemma 3.2. Then $a b=\sum\left(y_{i} z_{i}\right) b=\sum y_{i}\left(z_{i} b\right) \in \tilde{J}_{0}$. 
It remains to show that $M_{0}$ is isomorphic to the degree zero Hochschild homology of $\tilde{M}_{0}$. Now $M_{0}=\mathscr{A}_{0} / J_{0}$, where $J_{0}:=\mathbb{C}_{0} \otimes_{S} J$. We observe that the difference between $J_{0}$ and $\tilde{J}_{0}$ is that $J_{0}$ contains the linear span of $a b-b a$ for all $a, b \in \mathscr{A}_{0}$, which descends to the linear span of arbitrary commutators in the ring $\tilde{M}_{0}$.

Remark 3.9. The equivariant Hikita conjecture [KTW ${ }^{+} 19 \mathrm{a}$, Conjecture 8.9] states that, in the presence of symplectic duality, $B(\mathscr{A})$ is isomorphic to the equivariant cohomology ring of the dual variety, which is concentrated in even degree. If this conjecture holds, then $B(\mathscr{A})$ is commutative, and therefore equal to its own degree zero Hochschild homology. Thus, if we assume that $X$ has a symplectic dual for which the equivariant Hikita conjecture holds, then Proposition 3.8 simply says that $M_{0}$ is isomorphic to $B(\mathscr{A})$.

Now we go in the opposite direction and consider the $\mathbb{C}[\hbar]$-algebras

$$
M_{T}:=\mathcal{O}(T) \otimes_{S} M \quad \text { and } \quad M_{1}:=\mathbb{C}_{1} \otimes_{S} M
$$

obtained by specializing $M$ over the full torus $T$ and at the identity element of $T$.

Remark 3.10. We note that $M_{0}$ can be recovered from $M_{\text {reg }}$, since $1-q^{\lambda}$ does not evaluate to zero at the fixed point of Spec $S$. On the other hand, $M_{1}$ cannot be recovered from $M_{\text {reg }}$, since $1-q^{\lambda}$ does evaluate to zero at the identity element of $T$.

Consider the algebra $\mathscr{A}^{\hbar=1}:=\mathscr{A} \otimes_{\mathbb{C}[\hbar]} \mathbb{C}[\hbar] /\langle\hbar-1\rangle$. Define a bimodule over this algebra with underlying vector space $\mathcal{O}(T) \otimes \mathscr{A}^{\hbar=1}$, where the left action is given by $a \cdot f \otimes b=f q^{-\lambda} \otimes a b$ for $a \in \mathscr{A}_{\lambda}^{\hbar=1}, f \in \mathcal{O}(T)$, and $b \in \mathscr{A}^{\hbar=1}$, and where the right action is just given by right multiplication.

Remark 3.11. The definition of this bimodule is motivated by a recent paper by EtingofStryker [ES]. They study twisted traces, which are closely related to the graded traces we study in this paper.

Consider the $\mathscr{A}^{\hbar=1}$-modules

$$
M_{T}^{\hbar=1}:=\mathscr{A}^{\hbar=1} \otimes_{\mathscr{A}} M_{T} \quad \text { and } \quad M_{1}^{\hbar=1}:=\mathscr{A}^{\hbar=1} \otimes_{\mathscr{A}} M_{1} .
$$

Proposition 3.12. We have vector space isomorphisms

$$
M_{T}^{\hbar=1} \cong H H_{0}\left(\mathscr{A}^{\hbar=1}, \mathcal{O}(T) \otimes \mathscr{A}^{\hbar=1}\right) \quad \text { and } \quad M_{1}^{\hbar=1} \cong H H_{0}\left(\mathscr{A}^{\hbar=1}\right)
$$

Proof. We first observe that the second isomorphism follows from the first, so we only need 
to prove the first. Using the invertibility of $q^{\lambda}$ in $\mathcal{O}(T)$, we see that

$H H_{0}\left(\mathscr{A}^{\hbar=1}, \mathcal{O}(T) \otimes \mathscr{A}^{\hbar=1}\right)=\mathcal{O}(T) \otimes \mathscr{A}^{\hbar=1} / \sum_{\lambda \in t_{\mathbb{Z}}^{*}} \mathcal{O}(T)\left\{1 \otimes a b-q^{\lambda} \otimes b a \mid a \in \mathscr{A}_{\lambda}^{\hbar=1}, b \in \mathscr{A}^{\hbar=1}\right\}$.

Given $\lambda \neq 0$, choose $x \in \mathscr{A}_{0}^{2}$ such that $\langle\lambda, \bar{x}\rangle \neq 0$. If $b \in \mathscr{A}_{\lambda}^{\hbar=1}$, then the commutator of $b$ with the image of $x$ in $\mathscr{A}_{0}^{\hbar=1}$ will be a nonzero multiple of $b$, which implies that the image of $1 \otimes b$ in $H H_{0}\left(\mathscr{A}^{\hbar=1}, \mathcal{O}(T) \otimes \mathscr{A}^{\hbar=1}\right)$ will be trivial. It follows that $H H_{0}\left(\mathscr{A}^{\hbar=1}, \mathcal{O}(T) \otimes \mathscr{A}^{\hbar=1}\right)$ is a quotient of $\mathcal{O}(T) \otimes \mathscr{A}_{0}^{\hbar=1}$.

Let

$$
J_{T}^{\hbar=1}:=\sum_{\lambda \in \mathbb{N} \Sigma_{+}} \mathcal{O}(T)\left\{1 \otimes a b-q^{\lambda} \otimes b a \mid a \in \mathscr{A}_{\lambda}^{\hbar=1}, b \in \mathscr{A}_{-\lambda}^{\hbar=1}\right\} \subset \mathcal{O}(T) \otimes \mathscr{A}_{0}^{\hbar=1},
$$

so that $M_{T}^{\hbar=1}=\mathcal{O}(T) \otimes \mathscr{A}_{0}^{\hbar=1} / J_{T}^{\hbar=1}$. It is clear that $J_{T}^{\hbar=1}$ is contained in the kernel of the map

$$
\mathcal{O}(T) \otimes \mathscr{A}_{0}^{\hbar=1} \rightarrow H H_{0}\left(\mathscr{A}^{\hbar=1}, \mathcal{O}(T) \otimes \mathscr{A}^{\hbar=1}\right)
$$

so it suffices to prove the reverse containment.

The $\mathbb{N}$-grading on $\mathscr{A}$ descends to a filtration of $\mathscr{A}^{\hbar=1}$, where $\left(\mathscr{A}^{\hbar=1}\right)^{k}$ is the image of $\mathscr{A}^{\leq k}$ in $\mathscr{A}^{\hbar=1}$. We will show that, if $a \in\left(\mathscr{A}_{\mu}^{\hbar=1}\right)^{k}$ and $b \in\left(\mathscr{A}_{-\mu}^{\hbar=1}\right)^{l}$, then $1 \otimes a b-q^{\mu} \otimes b a \in J_{T}^{\hbar=1}$. We will assume without loss of generality that $k \leq l$ and proceed by induction on $k$.

Assume first that $\langle\mu, \xi\rangle>0$. As in Lemma 3.2, write $a=\sum y_{i} z_{i}$ for some $y_{i} \in \mathscr{A}_{\lambda_{i}}^{\hbar=1}$ and $z_{i} \in\left(\mathscr{A}_{\mu-\lambda_{i}}^{\hbar=1}\right)^{q_{i}}$ with $\lambda_{i} \in \Sigma_{+}$and $q_{i}<k$ for all $i$. Then for each $i$, we have

$$
1 \otimes y_{i} z_{i} b-q^{\mu} \otimes b y_{i} z_{i}=1 \otimes y_{i} z_{i} b-q^{\lambda_{i}} \otimes z_{i} b y_{i}+q^{\lambda_{i}}\left(1 \otimes z_{i} b y_{i}-q^{\mu-\lambda_{i}} \otimes b y_{i} z_{i}\right)
$$

By the definition of $J_{T}^{\hbar=1}$, we see that $1 \otimes y_{i} z_{i} b-q^{\lambda_{i}} \otimes z_{i} b y_{i} \in J_{T}^{\hbar=1}$. Our inductive hypothesis tells us that $1 \otimes z_{i} b y_{i}-q^{\mu-\lambda_{i}} \otimes b y_{i} z_{i} \in J_{T}^{\hbar=1}$, as well. Thus $1 \otimes y_{i} z_{i} b-q^{\mu} \otimes b y_{i} z_{i} \in J_{T}^{\hbar=1}$ for all $i$, and therefore $1 \otimes a b-q^{\mu} \otimes b a \in J_{T}^{\hbar=1}$ as desired.

If $\langle\mu, \xi\rangle<0$, then we can replace $\xi$ with $-\xi$, which does not affect the definitions of either $J_{T}^{\hbar=1}$ or $H H_{0}(A, \mathcal{O}(T) \otimes A)$, and thus reduce to the previous case. Finally, suppose that $\langle\mu, \xi\rangle=0$. If $\mu \neq 0$, then we can perturb $\xi$ so that this is no longer the case without changing $\Sigma_{+}$. This again does not affect the definitions of either $J_{T}^{\hbar=1}$ or $H H_{0}(A, \mathcal{O}(T) \otimes A)$, and we may again reduce to the previous cases. If $\mu=0$, then the fact that $1 \otimes a b-q^{\mu} \otimes b a=[a, b] \in J_{T}^{\hbar=1}$ is immediate from the definition of $J$. 


\subsection{Finite generation}

Recall that $M_{\text {reg }}$ is a module over $R_{\text {reg }}$, and $R_{\text {reg }}$ may be regarded as a subring of differential operators on Spec $S_{\text {reg }}$ with values in $H_{2}\left(\tilde{X}^{\mathrm{sm}} ; \mathbb{C}\right)$ (Remark 3.3). In particular $R_{\text {reg }}$ is generated by three types of elements: the "vector fields" $\mathfrak{t}$, the "functions" $S_{\text {reg }}$, and the "values" $H_{2}\left(\tilde{X}^{\mathrm{sm}} ; \mathbb{C}\right)$. The following result says that $M_{\text {reg }}$ is finitely generated over just the functions and the values.

Proposition 3.13. If $M_{0}$ is finitely generated as a module over $\operatorname{Sym} H_{2}\left(\tilde{X}^{\mathrm{sm}} ; \mathbb{C}\right) \otimes \mathbb{C}[\hbar]$, then $M_{\text {reg }}$ is finitely generated as a module over $S_{\text {reg }} \otimes \operatorname{Sym} H_{2}\left(\tilde{X}^{\mathrm{sm}} ; \mathbb{C}\right) \otimes \mathbb{C}[\hbar]$.

Proof. Choose elements $x_{1}, \ldots, x_{d} \in \mathscr{A}_{0}$ whose images generate $M_{0}$ as a module over $\operatorname{Sym}_{2}\left(\tilde{X}^{\text {sm }} ; \mathbb{C}\right) \otimes$ $\mathbb{C}[\hbar]$, and let $M_{\text {reg }}^{\prime} \subset M_{\text {reg }}$ be the submodule spanned by the images of $x_{1}, \ldots, x_{d}$ in $M_{\text {reg. }}$. We will show that $M_{\text {reg }}^{\prime}=M_{\text {reg. }}$. To do this, we will prove by induction that, for any natural number $m$, the degree $m$ parts of $M_{\text {reg }}$ and $M_{\text {reg }}^{\prime}$ coincide. The base case $m=0$ holds because $M_{\mathrm{reg}}^{0}=S_{\mathrm{reg}}$.

Let $a \in \mathscr{A}_{0}^{m}$. Since $x_{1}, \ldots, x_{d}$ generate $M_{0}$, we may choose elements $r_{1}, \ldots, r_{d} \in$ $\operatorname{Sym} H_{2}\left(\tilde{X}^{\mathrm{sm}} ; \mathbb{C}\right) \otimes \mathbb{C}[\hbar], \lambda_{1}, \ldots, \lambda_{e} \in \mathbb{N} \Sigma_{+}$, and $a_{1}, b_{1}, \ldots, a_{e}, b_{e} \in \mathscr{A}$ with $a_{j} \in \mathscr{A}_{\lambda_{j}}$ and $b_{j} \in \mathscr{A}_{-\lambda_{j}}$, such that

$$
a=\sum_{i=1}^{d} r_{i} x_{i}+\sum_{j=1}^{e} a_{j} b_{j} \in \mathscr{A}_{0}^{m} .
$$

By Lemma 3.2, we may also assume that $\lambda_{j} \in \Sigma_{+}$for all $j$. It now suffices to show that, for each $j$, the image of $a_{j} b_{j}$ in $M_{\text {reg }}$ lies in $M_{\text {reg }}^{\prime}$.

In $M_{\text {reg }}$, we have

$$
a_{j} b_{j}=q^{\lambda_{j}} b_{j} a_{j} .
$$

On the other hand, since the quotient of $\mathscr{A}$ by $\mathscr{A} \hbar$ is commutative, there exists an element $c \in \mathscr{A}_{0}^{m-2}$ such that $\left[b_{j}, a_{j}\right]=\hbar c$. Combining this with the previous equation, we see that in $M_{\text {reg }}$,

$$
a_{j} b_{j}=\frac{q^{\lambda_{j}}}{1-q^{\lambda_{j}}} \hbar c .
$$

By our inductive hypothesis, the image of $c$ lies in $M_{\mathrm{reg}}^{\prime}$, thus so does the image of $a_{j} b_{j}$.

By Proposition 3.8 and [BLPW16, Proposition 5.1], $M_{0}$ is finitely generated as a module over $\operatorname{Sym} H_{2}\left(\tilde{X}^{\mathrm{sm}} ; \mathbb{C}\right) \otimes \mathbb{C}[\hbar]$ whenever $\tilde{X}$ is smooth and the action of $T$ on $\tilde{X}$ has isolated fixed points. We thus have the following corollary.

Corollary 3.14. If $\tilde{X}$ is smooth and $T$ acts on $\tilde{X}$ with isolated fixed points, then $M_{\mathrm{reg}}$ is finitely generated as a module over $S_{\mathrm{reg}} \otimes \operatorname{Sym} H_{2}(\tilde{X} ; \mathbb{C}) \otimes \mathbb{C}[\hbar]$. 


\subsection{Traces}

Given an $\mathbb{C}$-algebra $A$, its degree zero Hochschild homology is used to study traces of finitedimensional representations. Indeed, if $V$ is an $A$-module which is finite-dimensional as a $\mathbb{C}$-vector space, then the trace map $A \rightarrow \mathbb{C}$ given by $a \mapsto \operatorname{tr}\left(\left.a\right|_{V}\right)$ factors through $H_{0}(A)$. Our algebra $\mathscr{A}$ carries a grading and the $R$-module $M$ can be thought of as a graded version of the degree zero Hoschshild homology of $\mathscr{A}$ (if we set $\hbar=1$ and restrict to $T$, this is Proposition $[3.12$ ). Now, we will define a notion of graded traces for $\mathscr{A}$-modules and prove that these graded traces factor through $M$ (Proposition 3.15).

Let $\Pi$ denote the set of linear maps $\mu: \mathscr{A}_{0}^{2} \rightarrow \mathbb{C} \hbar$ such that $\mu(\hbar)=\hbar$. There is a free action of $\mathfrak{t}_{\mathbb{Z}}^{*}$ on $\Pi$ given by $(\lambda+\mu)(x)=\mu(x)+\hbar \lambda(\bar{x})$, where $\lambda \in \mathfrak{t}_{\mathbb{Z}}^{*}$ and $\mu \in \Pi$. Let

$$
N:=\left\{\sum_{\mu \in \Pi} p_{\mu} q^{\mu} \mid p_{\mu} \in \mathbb{C}[\hbar]\right\} .
$$

Note that $N$ is similar to $S \otimes \mathbb{C}[\hbar]$, but it is much bigger; we allow the exponent of $q$ to live in $\Pi$ rather than in $\mathbb{N} \Sigma_{+}$, and we also allow infinite rather than finite sums. We endow $N$ with the structure of a left $R$-module by putting

$$
q^{\lambda} \cdot q^{\mu}=q^{\lambda+\mu} \quad \text { and } \quad x \cdot q^{\mu}=\mu(x) q^{\mu}
$$

for all $\lambda \in \mathbb{N} \Sigma_{+}, \mu \in \Pi$, and $x \in \mathscr{A}_{0}^{2}$.

Let $V$ be a graded $\mathscr{A}$-module. For any $\mu \in \Pi$, let

$$
V_{\mu}:=\left\{v \in V \mid x \cdot v=\mu(x) v \text { for all } x \in \mathscr{A}_{0}^{2}\right\}
$$

Then for all $a \in \mathscr{A}_{\lambda}$ and $v \in V_{\mu}$, we have $a \cdot v \in V_{\lambda+\mu}$. If each $V_{\mu}$ is a free $\mathbb{C}[\hbar]$-module of finite rank, we call $V$ reasonable. We define the graded trace of a reasonable $\mathscr{A}$-module $V$ to be the map

$$
\begin{aligned}
\operatorname{tr}_{V}: \mathscr{A}_{0} & \rightarrow N \\
a & \mapsto \sum_{\mu \in \Pi} \operatorname{tr}\left(\left.a\right|_{V_{\mu}}\right) q^{\mu} .
\end{aligned}
$$

In particular $\chi_{V}:=\operatorname{tr}_{V}(1)$ is the generating function for the ranks of the weight spaces as $\mathbb{C}[\hbar]$-modules, which we will refer to as the character of the representation.

Proposition 3.15. The graded trace descends to an $\mathbb{N}$-graded $R$-module map $\operatorname{tr}_{V}: M \rightarrow N$.

Proof. We need to show that $J$ is contained in the kernel of $\operatorname{tr}_{V}$. More concretely, if $a \in \mathscr{A}_{\lambda}$ and $b \in \mathscr{A}_{-\lambda}$, we need to show that $\operatorname{tr}_{V}(a b)=q^{\lambda} \operatorname{tr}_{V}(b a)$. Pick an element $\mu \in \Pi$ and consider the maps $a: V_{\mu} \rightarrow V_{\lambda+\mu}$ and $b: V_{\lambda+\mu} \rightarrow V_{\mu}$. Since these are linear maps between finite-rank 
free $\mathbb{C}[\hbar]$-modules, we have

$$
\operatorname{tr}\left(\left.b a\right|_{V_{\mu}}\right)=\operatorname{tr}\left(\left.a b\right|_{V_{\lambda+\mu}}\right)
$$

and therefore

$$
q^{\lambda} \operatorname{tr}_{V}(b a)=q^{\lambda} \sum_{\mu} \operatorname{tr}\left(\left.b a\right|_{V_{\mu}}\right) q^{\mu}=q^{\lambda} \sum_{\mu} \operatorname{tr}\left(\left.a b\right|_{V_{\lambda+\mu}}\right) q^{\mu}=\sum_{\mu} \operatorname{tr}\left(\left.a b\right|_{V_{\lambda+\mu}}\right) q^{\lambda+\mu}=\operatorname{tr}_{V}(a b) .
$$

This completes the proof.

Remark 3.16. Fix a splitting of the quantization exact sequence (2). Recall from Remark 3.3 that, given $c \in H^{2}\left(\tilde{X}^{\mathrm{sm}} ; \mathbb{C}\right)$, the ring $R_{T}^{c}$ obtained by localizing to $T$ and killing the ideal generated by $\theta-\hbar\langle\theta, c\rangle$ for all $\theta \in H_{2}\left(\tilde{X}^{\mathrm{sm}} ; \mathbb{C}\right)$ is isomorphic to the Rees algebra of the ring of differential operators on $T$. Let $M^{c}:=R^{c} \otimes_{R} M$,

$$
\Pi^{c}:=\left\{\mu \in \Pi \mid \mu(\theta)=\hbar\langle\theta, c\rangle \text { for all } \theta \in H_{2}\left(\tilde{X}^{\mathrm{sm}} ; \mathbb{C}\right)\right\},
$$

The splitting of (2) identifies $\Pi^{c}$ with $\mathfrak{t}^{*}$. Define

$$
N^{c}:=\left\{\sum_{\mu \in \Pi^{c}} p_{\mu} q^{\mu} \mid p_{\mu} \in \mathbb{C}[\hbar]\right\} .
$$

We endow $N^{c}$ with an $R^{c}$-module structure as above. Then for any reasonable $V$, the graded trace map descends to a $R^{c}$-module map

$$
\operatorname{tr}_{V}^{c}: M^{c} \rightarrow N^{c}
$$

We may regard this map as a "solution" to the D-module $M^{c}$ on $T$. Note that it depends on the choice of a splitting of (2), i.e. of a quantum co-moment map.

Remark 3.17. Let $N_{\text {reg }} \subset N$ be the set of all $\sum p_{\mu} q^{\mu}$ such that for all $\lambda \in \Sigma_{+}$and all $\mu \in \Pi$, we have $p_{\mu-n \lambda}=0$ for all $n \gg 0$. This is an $R$-submodule on which $1-q^{\lambda}$ acts invertibly for all $\lambda \in \Sigma_{+}$, and therefore the action of $R$ on $N_{\text {reg }}$ extends to an action of $R_{\text {reg. }}$. We say that a graded $\mathscr{A}$-module $V$ is positively reasonable if it is reasonable and $\operatorname{tr}_{V}(1) \in N_{\text {reg }}$.

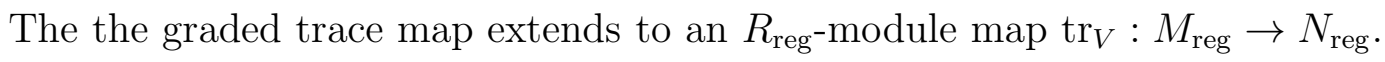

Example 3.18. We continue with Example 3.6. We have $\mathscr{A}_{0}^{2}=\mathbb{C}\left\{a_{1}, a_{2}, \hbar\right\}$. For any $\mu \in \Pi$, let $\mu_{i}:=\mu\left(a_{i}\right) / \hbar$, and write $q^{\mu}=q_{1}^{\mu_{1}} q_{2}^{\mu_{2}}$. Let $V$ be a positively reasonable representation of $\mathscr{A}$ on which the central element $a_{2}-a_{1} \in \mathscr{A}$ acts by a scalar $z \hbar$ for some complex number $z$; this implies that $V_{\mu}=0$ unless $\mu_{2}-\mu_{1}=z$. By Proposition 3.15, we have 
$a_{1} a_{2}(1-q) \chi_{V}=0 \in N$, which implies that there exist $p_{1}, p_{2} \in \mathbb{Z}$ such that

$$
(1-q) \chi_{V}=p_{1} q_{1}^{-z}+p_{2} q_{2}^{z}
$$

Then

$$
\chi_{V}=\frac{p_{1} q_{1}^{-z}+p_{2} q_{2}^{z}}{1-q}=p_{1} \sum_{n=0}^{\infty} q_{1}^{n-z} q_{2}^{n}+p_{2} \sum_{n=0}^{\infty} q_{1}^{n} q_{2}^{n+z},
$$

which is reminiscent of the Weyl character formula. If $V_{1}$ and $V_{2}$ are the Verma modules for $\mathscr{A}$ with central character $z \hbar$, we have

$$
\chi_{V_{1}}=\sum_{n=0}^{\infty} q_{1}^{n-z} q_{2}^{n} \quad \text { and } \quad \chi_{V_{2}}=\sum_{n=0}^{\infty} q_{1}^{n} q_{2}^{n+z} .
$$

If $z$ is a positive integer, there is a finite-dimension module $V$ with $p_{1}=1$ and $p_{2}=-1$, so that

$$
\chi_{V}=\sum_{n=0}^{z-1} q_{1}^{n-z} q_{2}^{n} .
$$

Similarly, if $z$ is a negative integer, there is a finite dimensional module $V$ with $p_{1}=-1$ and $p_{2}=1$, so that

$$
\chi_{V}=\sum_{n=0}^{1-z} q_{1}^{n} q_{2}^{n+z}
$$

\subsection{The rank of $M_{\text {reg }}$}

Assume for the remainder of this section that $X$ admits a $\left(T \times \mathbb{G}_{m}\right)$-equivariant projective symplectic resolution $\tilde{X}$ with isolated $T$-fixed points. For each fixed point $x \in \tilde{X}^{T}$, we may define a local version $\mathscr{A}_{x}$ of $\mathscr{A}$ by quantizing the tangent space of $x$. The inclusion of a formal neighborhood of $x$ into $\tilde{X}$ induces a $T$-equivariant surjection $\mathscr{A} \rightarrow \mathscr{A}_{x}$ [BLPW16, Section 5.1]. Given $c \in H^{2}(\tilde{X} ; \mathbb{C})$, let $\mathscr{A}_{x}^{c}$ be the quotient of $\mathscr{A}_{x}$ by the ideal generated by the central elements $\theta-\hbar\langle\theta, c\rangle$ for all $\theta \in H_{2}(\tilde{X} ; \mathbb{C})$. We note that $\mathscr{A}_{x}^{c}$ is isomorphic to the Rees algebra of a Weyl algebra.

We use the cocharacter $\xi \in \mathfrak{t}_{\mathbb{Z}}$ to induce a $\mathbb{Z}$-grading on $\mathscr{A}_{x}^{c}$, and denote by $\mathscr{A}_{x,-}^{c}$ the direct sum of the non-positive weight spaces. Consider the left $\mathscr{A}_{x}^{c}$-module

$$
\Theta_{x}^{c}:=\mathscr{A}_{x}^{c} \otimes_{\mathscr{A}_{x,-}^{c}} B\left(\mathscr{A}_{x}^{c}\right)
$$

Then $\Theta_{x}^{c}$ is positively reasonable in the sense of Remark 3.17 as a graded $\mathscr{A}$-module, and we have

$$
\operatorname{tr}_{\Theta_{x}^{c}}^{c}(1 \otimes 1)=q^{w_{x}^{c}} \prod_{i} \frac{1}{1-q^{\alpha_{i}}}
$$


where the elements $\alpha_{i} \in \Sigma_{+}$are the positive weights of the action of $T$ on the tangent space $T_{x} \tilde{X}$, and $w_{x}^{c} \in \Pi^{c}$ is the linear map $w_{x}^{c}: \mathscr{A}_{0}^{2} \rightarrow \mathbb{C} \hbar$ given by restricting

$$
\mathscr{A}_{0} \rightarrow\left(\mathscr{A}_{x}^{c}\right)_{0} \rightarrow B\left(\mathscr{A}_{x}^{c}\right) \cong \mathbb{C}[\hbar]
$$

to degree 2 [BLPW16, Proposition 5.20].

Let $M\left(\mathscr{A}_{x}\right):=S \otimes \mathscr{A}_{x, 0} / J_{x}$, where $J_{x}$ is defined exactly as in Equation (4)). Define $M\left(\mathscr{A}_{x}\right)^{c}:=R^{c} \otimes_{R} M\left(\mathscr{A}_{x}\right)$ as in Section [3.6, and define $M\left(\mathscr{A}_{x}\right)_{\text {reg }}$ and $M\left(\mathscr{A}_{x}\right)_{\text {reg }}^{c}$ in the obvious manner.

Lemma 3.19. The natural map

$$
r_{x}: S_{\mathrm{reg}} \otimes \mathbb{C}[\hbar] \rightarrow M\left(\mathscr{A}_{x}\right)_{\mathrm{reg}}^{c}
$$

is an isomorphism.

Proof. We begin by showing that $r_{x}$ is a surjection. The algebra $\mathscr{A}_{x}$ is generated over $\mathbb{C}[\hbar]$ by positive degree elements $z_{i}, w_{i}$ with weights $\alpha_{i},-\alpha_{i}$ and relations $\left[w_{i}, z_{j}\right]= \pm \delta_{i j} \hbar$. By induction, we suppose that all elements of $M\left(\mathscr{A}_{x}\right)_{\text {reg }}$ of degree strictly less than $d$ lie in the image of $r_{x}$. Let $m \in M\left(\mathscr{A}_{x}\right)_{\text {reg }}^{c}$ be an element of degree $d>0$. Then there exist elements $a_{i} \in \mathscr{A}_{x}$ of weight $-\alpha_{i}$ and degree strictly less than $d, m^{\prime} \in M\left(\mathscr{A}_{x}\right)_{\text {reg }}^{c}$ of degree $d-2$ and $s_{i} \in S_{\text {reg }}$ such that

$$
m=\hbar m^{\prime}+\sum s_{i} \otimes z_{i} a_{i}
$$

It will therefore suffice to show that each $1 \otimes z_{i} a_{i}$ lies in the image of $r_{x}$. We have

$$
1 \otimes z_{i} a_{i}=q^{\alpha_{i}} \otimes a_{i} z_{i}=q^{\alpha_{i}} \otimes\left(z_{i} a_{i}+\left[a_{i}, z_{i}\right]\right)
$$

which implies that

$$
\left(1-q^{\alpha_{i}}\right) \otimes z_{i} a_{i}=q^{\alpha_{i}} \otimes\left[a_{i}, z_{i}\right]
$$

and therefore

$$
1 \otimes z_{i} a_{i}=\frac{q^{\alpha_{i}}}{1-q^{\alpha_{i}}} \otimes\left[a_{i}, z_{i}\right]
$$

Since $\left[a_{i}, z_{i}\right]$ is a multiple of $\hbar$, our inductive hypothesis implies that this element lies in the image of $r_{x}$.

It remains to show that the kernel of $r_{x}$ is trivial. The composition

$$
\operatorname{tr}_{\Theta_{x}^{c}}^{c} \circ r_{x}: S_{\mathrm{reg}} \otimes \mathbb{C}[\hbar] \rightarrow N^{c}
$$

takes $s$ to $s \cdot \operatorname{tr}_{\Theta_{x}^{c}}^{c}(1)$, and this map is clearly injective by Equation (15). Thus $r_{x}$ must be injective, as well. 
Let $T_{\text {reg }}:=T \cap \operatorname{Spec} S_{\text {reg }}$, and let

$$
M_{T_{\text {reg }}}:=\mathcal{O}\left(T_{\text {reg }}\right) \otimes_{S} M
$$

Let $M^{c, 1}$ be the specialization of $M^{c}$ at $\hbar=1$, so that $M^{c}$ is isomorphic to the Rees module of the filtered module $M^{c, 1}$. Define $M_{T_{\mathrm{reg}}}^{c, 1}$ similarly. For the remainder of this section, we will fix a splitting of the quantization exact sequence, so that $M_{T_{\text {reg }}}^{c, 1}$ defines a $D\left(T_{\text {reg }}\right)$-module by Remark 3.3. This choice of splitting is not essential in any way, but it is convenient because it allows us to use the language of D-modules.

Since $M_{T_{\text {reg }}}^{c, 1}$ is finitely generated over $\mathcal{O}\left(T_{\text {reg }}\right)$ (Corollary 3.14), it defines a nonsingular D-module over $T_{\text {reg }}$, and therefore comes from a vector bundle with flat connection; we will use the same notation $M_{T_{\text {reg }}}^{c, 1}$ to refer to this vector bundle. The main result of this section, Corollary 3.24, computes the rank of this vector bundle under certain assumptions.

We will say that $c$ is generic if it satisfies the conditions of [BLPW16, Theorem 5.12] and [BLPW16, Lemma 5.21] for each fixed point $x \in \tilde{X}^{T}$.

Proposition 3.20. If $c$ is generic, then $\operatorname{rk} M_{T_{\mathrm{reg}}}^{c, 1} \leq\left|\tilde{X}^{T}\right|$.

Proof. We have a coherent sheaf $M^{c, 1}$ on Spec $S$ whose restriction to $T_{\text {reg }}$ is equal to the vector bundle $M_{T_{\text {reg }}}^{c, 1}$, thus the rank of this vector bundle is bounded above by the dimension of the fiber of $M^{c, 1}$ at the point $0 \in \operatorname{Spec} S$. By Proposition 3.8, this fiber is isomorphic to $H H_{0}(B(\mathscr{A}))^{c, 1}$, which is a quotient of $B(\mathscr{A})^{c, 1}$. For generic $c$, the dimension of $B(\mathscr{A})^{c, 1}$ is equal to $\left|\tilde{X}^{T}\right|$ by [BLPW16, Proposition 5.3].

Our splitting of the quantization exact sequence identifies $\Pi^{c}$ with $\mathfrak{t}^{*}$, thus $q^{w_{x}^{c}}$ defines a multi-valued function on $T_{\text {reg }}$ with monodromy $e^{2 \pi i w_{x}^{c}(\tau)}$ around any loop $\tau \in \pi_{1}(T) \cong \mathfrak{t}_{\mathbb{Z}}$. Consider the rank one D-module $\mathcal{O}\left(T_{\text {reg }}\right) q^{w_{x}^{c}}$ generated by $q^{w_{x}^{c}}$. In other words, it is the quotient of $D\left(T_{\text {reg }}\right)$ by the left ideal $\left\langle\partial_{u}-w_{x}^{c}(u)\right\rangle$ for $u \in \mathfrak{t}$. Equation (5) and Lemma 3.19 together imply that the map $\operatorname{tr}_{\Theta_{x}^{c}}^{c}: M^{c} \rightarrow N^{c}$ descends to a nonzero map

$$
\operatorname{tr}_{\Theta_{x}^{c}}^{c, 1}: M_{T_{\mathrm{reg}}}^{c, 1} \rightarrow \mathcal{O}\left(T_{\mathrm{reg}}\right) q^{w_{x}^{c}} .
$$

Since the target is a simple $D$-module, it must be surjective.

Consider the sum

$$
\theta^{c}: M_{T_{\mathrm{reg}}}^{c, 1} \rightarrow \bigoplus_{x \in \tilde{X}^{T}} \mathcal{O}\left(T_{\mathrm{reg}}\right) q^{w_{x}^{c}}
$$

of these maps over all fixed points. If the cosets $w_{x}^{c}+\mathfrak{t}_{\mathbb{Z}}^{*}$ are distinct, then the summands of the target are non-isomorphic simple $D$-modules and the map $\theta^{c}$ must therefore be surjective. We will show that, under additional hypotheses, this is the case. Specifically, in Appendix ఏ we define maps $\rho_{x, y}^{*}: H^{2}(\tilde{X} ; \mathbb{C}) \rightarrow \mathfrak{t}^{*}$ for any pair of fixed points $x, y \in \tilde{X}^{T}$, and we 
ask that these maps be nonzero for $x \neq y \in \tilde{X}^{T}$. This holds, for instance, for hypertoric varieties attached to unimodular arrangements and for the Springer resolution, but not for the balanced Hilbert scheme of $n$ points in the plane.

Lemma 3.21. Suppose that the map $\rho_{x, y}$ in nonzero for all $x \neq y \in \tilde{X}^{T}$. For $c$ in $a$ non-empty analytic open subset, the cosets $w_{x}^{c}+\mathfrak{t}_{\mathbb{Z}}^{*}$ are distinct.

Proof. For any two fixed points $x \neq y \in \tilde{X}^{T}$, Theorem A.3 (with $\hbar=1$ ) tells us that

$$
w_{x}^{c}-w_{y}^{c}=\rho_{x, y}^{*}(c)+\chi_{x}-\chi_{y}
$$

where $\chi_{x} \in \mathfrak{t}^{*}$ is independent of $c$. Since we require $\rho_{x, y}$ to be nonzero, the set of $c$ for which any one of these differences lies in $\mathfrak{t}_{\mathbb{Z}}^{*}$ forms a discrete union of affine subspaces of codimension $\geq 1$.

Proposition 3.22. Suppose that the map $\rho_{x, y}$ in nonzero for all $x \neq y \in \tilde{X}^{T}$. For all $c \in H^{2}(\tilde{X} ; \mathbb{C})$, we have

$$
\operatorname{rk} M_{T_{\mathrm{reg}}}^{c, 1} \geq\left|\tilde{X}^{T}\right| .
$$

Proof. Since $M_{T_{\text {reg }}}$ is coherent over $H^{2}(\tilde{X} ; \mathbb{C}) \times T_{\text {reg }} \times \operatorname{Spec} \mathbb{C}[\hbar]$, it is enough to prove this for $c$ lying in a nonempty analytic open subset. By Lemma 3.21, there exists such a subset for which the cosets $w_{x}^{c}+\mathfrak{t}_{\mathbb{Z}}^{*}$ are distinct. For $c$ in this subset, the map $\theta^{c}$ must be surjective, thus $\operatorname{rk} M_{T_{\text {reg }}}^{c, 1} \geq\left|\tilde{X}^{T}\right|$.

Since $M_{\text {reg }}^{c, 1}$ is coherent over Spec $S_{\text {reg }}$ and $T_{\text {reg }}$ is dense in Spec $S_{\text {reg }}$, we have the following mild strengthening of Proposition 3.22 .

Corollary 3.23. Suppose that the maps $\rho_{x, y}$ are nonzero for all $x \neq y \in \tilde{X}^{T}$. For all $c$ and any $q \in \operatorname{Spec} S_{\mathrm{reg}}$, the fiber of $M_{\text {reg }}^{c, 1}$ at $q$ has dimension greater than or equal to $\left|\tilde{X}^{T}\right|$.

Combining Propositions 3.20 and 3.22 , we obtain the main result of this section.

Corollary 3.24. Suppose that the map $\rho_{x, y}$ in nonzero for all $x \neq y \in \tilde{X}^{T}$. For $c \in H^{2}(\tilde{X} ; \mathbb{C})$ generic, $\mathrm{rk} M_{T_{\mathrm{reg}}}^{c, 1}=\left|\tilde{X}^{T}\right|$, the map $\theta^{c}$ is an isomorphism, and thus we have an isomorphism of D-modules

$$
M_{T_{\mathrm{reg}}}^{c, 1} \cong \bigoplus_{x \in \tilde{X}^{T}} \mathcal{O}\left(T_{\mathrm{reg}}\right) q^{w_{x}^{c}} .
$$

Remark 3.25. Many interesting symplectic resolutions $\tilde{X}$ with isolated fixed-points, such as $\tilde{X}=\operatorname{Hilb}_{n}\left(\mathbb{C}^{2}\right)$, do not have distinct restriction maps $H_{T}^{2}(\tilde{X} ; \mathbb{C}) \rightarrow H_{T}^{2}(x ; \mathbb{C})$. In this case the maps $\rho_{x, y}$ defined in the appendix vanish. On the other hand, the arguments in this section can be adapted to situations where there exists an element $m \in M_{\text {reg }}$ such that the functions $\operatorname{tr}_{\Theta_{x}^{c}}^{c}(m)$ are linearly independent. The case where $\tilde{X}$ has non-isolated fixed points is more mysterious. 


\section{Geometric construction}

We again fix a conical symplectic singularity $X$ as in Section 2, and we now assume that $X$ admits a $\left(T \times \mathbb{G}_{m}\right)$-equivariant projective symplectic resolution $\tilde{X}$, which we fix throughout this section. The odd cohomology of $\tilde{X}$ vanishes [BPW16, Proposition 2.5], thus we have a short exact sequence

$$
0 \rightarrow H_{T \times \mathbb{G}_{m}}^{2}(p t ; \mathbb{C}) \rightarrow H_{T \times \mathbb{G}_{m}}^{2}(\tilde{X} ; \mathbb{C}) \rightarrow H^{2}(\tilde{X} ; \mathbb{C}) \rightarrow 0
$$

which we will call the cohomology exact sequence. Given $u \in H_{T \times \mathbb{G}_{m}}^{2}(\tilde{X} ; \mathbb{C})$, let $\bar{u}$ denote its image in $H^{2}(\tilde{X} ; \mathbb{C})$.

\subsection{Quantum cohomology}

Let $H_{2}(\tilde{X} ; \mathbb{Z})_{\text {free }}$ denote the quotient of $H_{2}(\tilde{X} ; \mathbb{Z})$ by its torsion subgroup. Let $Q H_{T \times \mathbb{G}_{m}}^{*}(\tilde{X} ; \mathbb{C})$ be the equivariant quantum cohomology ring of $\tilde{X}$, with the quantum product shifted by the canonical theta characteristic. The underlying graded vector space of $Q H_{T \times \mathbb{G}_{m}}^{*}(\tilde{X} ; \mathbb{C})$ is equal to the tensor product of $H_{T \times \mathbb{G}_{m}}^{*}(\tilde{X} ; \mathbb{C})$ with the completion of the semigroup ring of the semigroup of effective curve classes in $H_{2}(\tilde{X} ; \mathbb{Z})_{\text {free }}$. Let $\star$ denote the quantum product and let $\hbar \in H_{T \times \mathbb{G}_{m}}^{*}(p t ; \mathbb{C})$ be the weight of the symplectic form. In [Oko15, Section 2.3.4], Okounkov conjectures that there exists a finite set $\Delta_{+} \subset H_{2}(\tilde{X} ; \mathbb{Z})_{\text {free }}$ and an element $L_{\alpha} \in$ $H^{2 \operatorname{dim} X}\left(\tilde{X} \times_{X} \tilde{X} ; \mathbb{C}\right)$ for each $\alpha \in \Delta_{+}$such that, for all $u \in H_{T \times \mathbb{C} \times}^{2}(\tilde{X} ; \mathbb{C})$,

$$
u \star \cdot=u \cup \cdot+\hbar \sum_{\alpha \in \Delta_{+}}\langle\alpha, \bar{u}\rangle \frac{q^{\alpha}}{1-q^{\alpha}} L_{\alpha}(\cdot),
$$

where $L_{\alpha}$ acts via convolution. We will assume that this conjecture holds. The minimal such subset $\Delta_{+}$is called the set of positive Kähler roots, and the set $\Delta:=\Delta_{+} \cup-\Delta_{+}$is called the set of Kähler roots. We will further assume that $\Delta_{+}$spans the lattice $H_{2}(\tilde{X} ; \mathbb{Z})_{\text {free }}$.

Let

$$
F:=\mathbb{C}\left\{q^{\alpha} \mid \alpha \in \mathbb{N} \Delta_{+}\right\} \quad \text { and } \quad F_{\text {reg }}:=F\left[\frac{1}{1-q^{\alpha}} \mid \alpha \in \Delta_{+}\right] .
$$

Our assumption that $\Delta_{+}$spans $H_{2}(\tilde{X} ; \mathbb{Z})_{\text {free }}$ implies that Spec $F$ contains the Kähler torus $K:=H^{2}\left(\tilde{X}, \mathbb{C}^{\times}\right)$as a dense open subset. Our assumptions also imply that quantum multiplication by a divisor restricts to an operator on the graded vector space

$$
Q_{\text {reg }}:=F_{\text {reg }} \otimes H_{T \times \mathbb{G}_{m}}^{*}(\tilde{X} ; \mathbb{C}),
$$

where $F_{\text {reg }}$ lives in degree zero. 
Remark 4.1. If the quantum cohomology $\operatorname{ring} Q H_{T \times \mathbb{G}_{m}}^{*}(\tilde{X} ; \mathbb{C})$ is generated by divisors, then our assumption implies that $Q_{\text {reg }}$ is a subring of the quantum cohomology ring. In general, however, we do not know that $Q_{\text {reg }}$ is a ring, as we make no assumptions about quantum multiplication by classes in degree greater than two.

\subsection{The specialized quantum D-module}

Let

$$
E:=F \otimes \operatorname{Sym} H_{T \times \mathbb{G}_{m}}^{2}(\tilde{X} ; \mathbb{C}),
$$

with multiplication defined by the formula

$$
u q^{\alpha}=q^{\alpha}(u+\hbar\langle\alpha, \bar{u}\rangle)
$$

for all $\alpha \in \mathbb{N} \Delta_{+}$and $u \in \operatorname{Sym} H_{T \times \mathbb{G}_{m}}^{2}(\tilde{X} ; \mathbb{C})$. We also let $E_{\text {reg }}$ be the Ore localization of $E$ with respect to the multiplicative subset generated by $\left(1-q^{\alpha}\right)$ for $\alpha \in \Delta_{+}$, which is well-defined by the same argument as in Lemma 3.4. The algebra $E_{\text {reg }}$ acts in a natural way on $Q_{\text {reg }}=F_{\text {reg }} \otimes H_{T \times \mathbb{G}_{m}}^{*}(\tilde{X} ; \mathbb{C})$. The subring $F_{\text {reg }} \subset E_{\text {reg }}$ acts by multiplication on the first tensor factor, while an element $u \in H_{T \times \mathbb{G}_{m}}^{2}(\tilde{X} ; \mathbb{C})$ acts by the operator $\hbar \partial_{\bar{u}}-u \star$ where $\partial_{\bar{u}}$ is the $K$-equivariant vector field on Spec $F$ defined by the equation $\partial_{\bar{u}}\left(q^{\alpha}\right)=\langle\alpha, \bar{u}\rangle q^{\alpha}$.

Remark 4.2. Just as we defined $R_{T}^{c}$ by specializing $H_{2}(\tilde{X} ; \mathbb{C}) \subset \mathscr{A}_{0}^{2}$ and localizing from Spec $S$ to $T$ (Remark 3.3), we also define $E_{K}^{c}$ for any $c \in \mathfrak{t}$ by specializing $\mathfrak{t}^{*} \subset H_{T \times \mathbb{G}_{m}}^{2}(\tilde{X} ; \mathbb{C})$ and localizing from $\operatorname{Spec} F$ to $K$. Then $E_{K}^{c}$ is non-canonically isomorphic to the Rees algebra of differential operators on $K$. If we further localize to $K_{\text {reg }}:=K \cap \operatorname{Spec} F_{\text {reg, }}$, we obtain the Rees algebra $E_{K_{\text {reg }}}^{c}$ of differential operators on $K_{\text {reg }}$ acting on $\mathcal{O}\left(K_{\text {reg }}\right) \otimes H_{\mathbb{G}_{m}}^{*}(\tilde{X} ; \mathbb{C})$.

Remark 4.3. In our construction, the ring $\mathbb{C}[\hbar]$ plays two a priori unrelated roles. It is identified both with the subring of $E$ generated by the Rees parameter, and with the ring of equivariant parameters $H_{\mathbb{G}_{m}}^{2}(p t ; \mathbb{C})$ acting on $H_{T \times \mathbb{G}_{m}}^{*}(\tilde{X} ; \mathbb{C})$. There is a more general construction in which one does not make this identification. Let

$$
\mathfrak{E}:=F \otimes \operatorname{Sym} H_{T \times \mathbb{G}_{m}}^{2}(\tilde{X} ; \mathbb{C}) \otimes \mathbb{C}[z]
$$

be the algebra with relations $u q^{\alpha}=q^{\alpha}(u+z\langle\alpha, \bar{u}\rangle)$, containing the central subalgebra $\mathbb{C}[z, \hbar]$; we then have $E \cong \mathfrak{E} /(z-\hbar) \mathfrak{E}$. The ring $\mathfrak{E}$ and its localization $\mathfrak{E}_{\text {reg }}$ act in a natural way on

$$
\mathfrak{Q}_{\mathrm{reg}}:=\mathcal{O}\left(K_{\mathrm{reg}}\right) \otimes H_{T \times \mathbb{G}_{m}}^{*}(\tilde{X} ; \mathbb{C}) \otimes \mathbb{C}[z]
$$

and we have $Q_{\text {reg }} \cong \mathfrak{Q}_{\text {reg }} /(z-\hbar) \mathfrak{Q}_{\text {reg }}$. The $\mathfrak{E}_{\text {reg-module }} \mathfrak{Q}_{\text {reg }}$ is traditionally called the quantum D-module. Thus our construction is a specialization of the quantum D-module, 
sometimes called the Calabi-Yau specialization. This specialization is often quite drastic: in many known cases, the monodromy of the quantum D-module becomes trivial at $z=\hbar$. Although the module $\mathfrak{Q}_{\text {reg }}$ motivates our definition of $Q_{\text {reg }}$, it will play no further role in this paper.

Remark 4.4. The advantage of working over Spec $F_{\text {reg }}$ rather than over $K_{\text {reg }}$ is that it makes sense to set $q$ equal to zero. The specialization $Q_{0}:=\mathbb{C}_{0} \otimes_{F_{\text {reg }}} Q_{\text {reg }}$ is a module over $\operatorname{Sym} H_{T \times \mathbb{G}_{m}}^{2}(\tilde{X} ; \mathbb{C})$, and it is canonically isomorphic to $H_{T \times \mathbb{G}_{m}}^{*}(\tilde{X} ; \mathbb{C})$.

Example 4.5. Continuing from Examples 3.6 and 3.18, suppose that $X$ is the Kleinian singularity of type $A_{1}$, in which case $\tilde{X} \cong T^{*} \mathbb{P}^{1}$. We may choose a basis $\hbar, u_{1}, u_{2}$ for $H_{T \times \mathbb{G}_{m}}^{2}(\tilde{X} ; \mathbb{C})$ such that $\bar{u}_{1}=\bar{u}_{2}$ and the classical cohomology ring is

$$
H_{T \times \mathbb{G}_{m}}^{*}(\tilde{X} ; \mathbb{C}) \cong \mathbb{C}\left[u_{1}, u_{2}, \hbar\right] /\left\langle u_{1} u_{2}\right\rangle
$$

In quantum cohomology, we have

$$
u_{1} \star u_{2}=\frac{\hbar q}{1-q}\left(\hbar+u_{1}+u_{2}\right)=q\left(\hbar+u_{1}\right) \star\left(\hbar+u_{2}\right) .
$$

This implies that

$$
Q H_{T \times \mathbb{G}_{m}}^{*}(\tilde{X} ; \mathbb{C}) \cong \mathbb{C}\left[u_{1}, u_{2}, \hbar\right][[q]] /\left\langle u_{1} \star u_{2}-q\left(\hbar+u_{1}\right) \star\left(\hbar+u_{2}\right)\right\rangle
$$

and

$$
Q_{\mathrm{reg}} \cong \mathbb{C}\left[u_{1}, u_{2}, \hbar, q,(1-q)^{-1}\right] /\left\langle u_{1} \star u_{2}-q\left(\hbar+u_{1}\right) \star\left(\hbar+u_{2}\right)\right\rangle .
$$

This is a module over $E_{\text {reg}}$, which is generated over $\mathbb{C}[\hbar]$ by $u_{1}, u_{2}, q$, and $(1-q)^{-1}$, with $\left[u_{1}, u_{2}\right]=0$ and $u_{i} q=q\left(u_{i}+\hbar\right)$. The element $q$ acts on $Q_{\text {reg }}$ by scalar multiplication and $u_{i}$ acts by $\hbar \partial-u_{i} \star$, where $\partial$ is the vector field given by $\partial(q)=q$.

\section{Duality}

In this section we formulate our main conjecture, which says that the modules constructed in Sections 3 and 4 are swapped under symplectic duality.

\subsection{Symplectic duality}

Let $X^{!}$be symplectic dual to $X$ in the sense of [BLPW16, Section 10.3]. We assume that $X^{!}$admits a symplectic resolution $\tilde{X}^{!}$, but we make no such assumption about $X$. We will not review the full definition of symplectic duality here, but rather focus on certain 
manifestations of this relationship and specific examples of dual pairs, which we list below. Our notational convention will be to denote everything related to $X^{!}$with a superscript. For example, $T^{!}$is the maximal torus of $\operatorname{Aut}\left(X^{!}\right)$, and so on.

- The most important aspect of the relationship between $X$ and $X^{!}$is that the quantization exact sequence (2) for $X$ is isomorphic to the cohomology exact sequence (6) for $X^{!}$. That is, we have isomorphisms $H_{2}\left(\tilde{X}^{\mathrm{sm}} ; \mathbb{C}\right) \cong\left(\mathfrak{t}^{!}\right)^{*}, \mathscr{A}_{0}^{2} \cong H_{T^{!} \times \mathbb{G}_{m}}^{2}\left(\tilde{X}^{!} ; \mathbb{C}\right)$, and $\mathfrak{t} \cong H^{2}\left(\tilde{X}^{!} ; \mathbb{C}\right)$, compatible with the maps 3 Moreover, we assume that this last isomorphism restricts to a lattice isomorphism $\mathfrak{t}_{\mathbb{Z}}^{*} \cong H_{2}\left(\tilde{X}^{!} ; \mathbb{Z}\right)$, which induces an isomorphism $T \cong K^{!}$.

- In Section [3.2, we had to choose a generic vector $\xi \in \mathfrak{t}_{\mathbb{Z}} \subset \mathfrak{t}_{\mathbb{R}} \cong H^{2}\left(\tilde{X}^{!} ; \mathbb{R}\right)$ that does not vanish on any of the equivariant roots of $X$. It is expected that the first Chern class of any ample line bundle on $\tilde{X}^{!}$will have this property, and that with this choice the positive equivariant roots for $X$ will coincide with the positive Kähler roots for $X^{!}$[Oko15, Section 3.1.8] (see also the coincidence of the twisting and shuffling arrangements in [BLPW16, Definition 10.1]). We will assume that this is the case. In particular, this implies that the rings $S$ and $F^{!}$are canonically identified, leading to an isomorphism of toric varieties $\operatorname{Spec} S \cong \operatorname{Spec} F^{!}$extending the above isomorphism of tori. Moreover, this implies a graded ring isomorphism

$$
R=S \otimes \operatorname{Sym} \mathscr{A}_{0}^{2} \cong F^{!} \otimes H_{T^{!} \times \mathbb{G}_{m}}^{2}\left(\tilde{X}^{!} ; \mathbb{C}\right)=E^{!}
$$

The main examples of dual pairs that we will consider in this paper are hypertoric varieties, which are dual to other hypertoric varieties, and the nilpotent cone in $\mathfrak{g}^{*}$ for a semisimple Lie algebra $\mathfrak{g}$, which is dual to the nilpotent cone in $\left(\mathfrak{g}^{!}\right)^{*}$, where $\mathfrak{g}^{!}$is the Langlands dual of $\mathfrak{g}$. Given an inclusion of groups $G \subset \tilde{G}$ and a representation $V$ of $\tilde{G}$, one can construct the Coulomb and Higgs branches of the associated gauge theory; when they are both conical symplectic singularities, they are expected to be symplectic dual. This class of examples includes hypertoric varieties (the case where $\tilde{G}$ is abelian) as well as the nilpotent cone in $\mathfrak{s l}_{n}^{*}$.

\subsection{Main conjecture}

Let $X$ and $X^{!}$be symplectic dual.

\footnotetext{
${ }^{3}$ The existence of such isomorphisms appears in BLPW16, Definition 10.15]. A choice of isomorphism $\mathscr{A}_{0}^{2} \cong H_{T^{!} \times \mathbb{G}_{m}}^{2}\left(\tilde{X}^{!} ; \mathbb{C}\right)$ was not previously considered to be part of the data of symplectic duality, but the examples that we consider here all come with a natural such choice.
} 
Conjecture 5.1. There is an isomorphism $M_{\mathrm{reg}} \cong Q_{\mathrm{reg}}^{!}$of graded modules over $R_{\mathrm{reg}} \cong E_{\mathrm{reg}}^{!}$ taking $1 \in M_{\text {reg }}$ to $1 \in Q_{\text {reg }}^{\text {! }}$.

We will prove that this conjecture holds for hypertoric varieties (Theorem 6.13) and for the Springer resolution (Theorem 7.17).

Remark 5.2. Choose $c \in H^{2}\left(\tilde{X}^{\text {sm }} ; \mathbb{C}\right) \cong \mathfrak{t}^{!}$and choose a splitting for the quantization exact sequence (2) for $X$, which is isomorphic by assumption to the cohomology exact sequence (16) for $X^{!}$. As in Remarks 3.3 and 4.2, we obtain an isomorphism between $R_{T_{\text {reg }}}^{c} \cong\left(E_{K_{\text {reg }}}^{!}\right)^{c}$ and the Rees algebra of differential operators on $T_{\text {reg }} \cong K_{\text {reg. }}^{!}$Thus Conjecture 5.1 becomes an isomorphism between modules over this ring of differential operators.

Remark 5.3. Proposition 3.8, Remark 4.4, and Conjecture 5.1 together imply that we have an isomorphism

$$
H H_{0}(B(\mathscr{A})) \cong M_{0} \cong Q_{0}^{!} \cong H_{T^{!} \times \mathbb{G}_{m}}^{*}\left(\tilde{X}^{!} ; \mathbb{C}\right)
$$

of graded modules over $\operatorname{Sym} \mathscr{A}_{0}^{2} \cong \operatorname{Sym} H_{T^{!} \times \mathbb{G}_{m}}^{2}(\tilde{X} ! \mathbb{C})$. If we further assume that $B(\mathscr{A})$ is commutative, which is true in all known examples, then we obtain an isomorphism $B(\mathscr{A}) \cong H_{T^{!} \times \mathbb{G}_{m}}^{*}\left(\tilde{X}^{!} ; \mathbb{C}\right)$. This is closely related to Nakajima's equivariant Hikita conjecture $\left[\mathrm{KTW}^{+} 19 \mathrm{a}\right.$, Conjecture 8.9$]$, which states that $B(\mathscr{A})$ and $H_{T^{!} \times \mathbb{G}_{m}}^{*}\left(\tilde{X}^{!} ; \mathbb{C}\right)$ are isomorphic as graded rings. In cases where $H_{T^{!} \times \mathbb{G}_{m}}^{*}\left(\tilde{X}^{!} ; \mathbb{C}\right)$ is generated in degree 2 , such as the hypertoric and Springer cases considered in this paper, the two statements are in fact equivalent.

Remark 5.4. We know that $Q_{\text {reg }}^{!}=F_{\text {reg }}^{!} \otimes H_{T^{!} \times \mathbb{G}_{m}}^{*}\left(\tilde{X}^{!} ; \mathbb{C}\right)$ is finitely generated over the ring $F_{\text {reg }}^{!} \otimes H_{T^{!} \times \mathbb{G}_{m}}^{*}(p t ; \mathbb{C})$, which is isomorphic to $S_{\text {reg }} \otimes \operatorname{Sym}_{2}\left(\tilde{X}^{\mathrm{sm}} ; \mathbb{C}\right) \otimes \mathbb{C}[\hbar]$. Thus Conjecture 5.1 would imply that $M_{\text {reg }}$ is finitely generated over the same ring. Assuming the equivariant Hikita conjecture $M_{0} \cong Q_{0}^{!}$(Remark 5.3), we know that $M_{0}$ is finitely generated over $H_{T^{!} \times \mathbb{G}_{m}}^{*}(p t ; \mathbb{C}) \cong \operatorname{Sym} H_{2}\left(\tilde{X}^{\mathrm{sm}} ; \mathbb{C}\right) \otimes \mathbb{C}[\hbar]$. Proposition 3.13 then implies that $M_{\text {reg }}$ is indeed finitely generated over $S_{\text {reg }} \otimes \operatorname{Sym} H_{2}\left(\tilde{X}^{\mathrm{sm}} ; \mathbb{C}\right) \otimes \mathbb{C}[\hbar]$. Thus Proposition 3.13 may be regarded as supporting evidence for Conjecture 5.1.

\section{$5.3 \quad$ Weyl groups}

The Namikawa Weyl group [Nam10] of $X$ is a finite group equipped with a faithful action on $H_{2}\left(\tilde{X}^{\mathrm{sm}} ; \mathbb{C}\right)$. As part of the package of symplectic duality, $W$ is identified with the Weyl group of the reductive group $\operatorname{Aut}\left(X^{!}\right)$in a manner compatible with the actions on $H_{2}\left(\tilde{X}^{\mathrm{sm}} ; \mathbb{C}\right) \cong\left(\mathfrak{t}^{!}\right)^{*}$. These actions extend to actions on $\mathscr{A}_{0}^{2}$ and $H_{T^{!} \times \mathbb{G}_{m}}^{2}\left(\tilde{X}^{!} ; \mathbb{C}\right)$ and then to the rings $R_{\text {reg }}$ and $E_{\text {reg }}^{!}$(acting trivially on $S_{\text {reg }}$ and $F_{\text {reg }}$ ). Moreover, $W$ acts compatibly on the modules $M_{\text {reg }}$ and $Q_{\text {reg }}^{!}$, and it is natural to expect the isomorphism of Conjecture 5.1 to 
be $W$-equivariant. In particular, this would imply that the $W$-invariant parts are isomorphic, as we conjecture below.

Conjecture 5.5. There is an isomorphism $M_{\mathrm{reg}}^{W} \cong\left(Q_{\mathrm{reg}}^{!}\right)^{W}$ of graded modules over $R_{\mathrm{reg}}^{W} \cong$ $\left(E_{\mathrm{reg}}^{!}\right)^{W}$ taking $1 \in M_{\text {reg }}^{W}$ to $1 \in\left(Q_{\text {reg }}^{!}\right)^{W}$.

Let us examine the objects appearing in Conjecture 5.5 for future use. On the algebraic side, we have $\mathscr{A} \cong \mathscr{A}^{W} \otimes_{\left(\operatorname{Sym} H_{2}\left(\tilde{X}^{\mathrm{sm}} ; \mathbb{C}\right)\right)^{W}} \operatorname{Sym} H_{2}\left(\tilde{X}^{\mathrm{sm}} ; \mathbb{C}\right)$ [LoS, Proposition 3.5$]$ and therefore

$$
J \cong J\left(\mathscr{A}^{W}\right) \otimes_{\left(\operatorname{Sym} H_{2}\left(\tilde{X}^{\mathrm{sm}} ; \mathbb{C}\right)\right)^{W}} \operatorname{Sym} H_{2}\left(\tilde{X}^{\mathrm{sm}} ; \mathbb{C}\right)
$$

where

$$
J\left(\mathscr{A}^{W}\right)=\sum_{\lambda \in \mathbb{N} \Sigma_{+}} S[\hbar] \cdot\left\{1 \otimes a b-q^{\lambda} \otimes b a \mid a \in \mathscr{A}_{\lambda}^{W}, b \in \mathscr{A}_{-\lambda}^{W}\right\} .
$$

This implies that

$$
M_{\mathrm{reg}}^{W}=\left(S_{\mathrm{reg}} \otimes \mathscr{A}_{0} / J\right)^{W} \cong S_{\mathrm{reg}} \otimes \mathscr{A}_{0}^{W} / J\left(\mathscr{A}^{W}\right) .
$$

In other words, $M_{\text {reg }}^{W}$ is obtained by applying our construction of the module $M_{\text {reg }}$ to the invariant algebra $\mathscr{A}^{W}$. On the other hand, since $\mathscr{A}$ is obtained from $\mathscr{A}^{W}$ by extension of scalars, we have

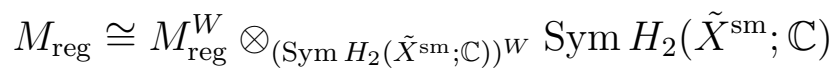

as $R_{\text {reg-modules. }}$

On the geometric side, we have

$$
\left(Q_{\mathrm{reg}}^{!}\right)^{W} \cong F_{\mathrm{reg}} \otimes H_{T^{!} \times \mathbb{G}_{m}}^{*}\left(\tilde{X}^{!} ; \mathbb{C}\right)^{W} \cong F_{\mathrm{reg}} \otimes H_{\mathrm{Aut}\left(\tilde{X}^{!}\right) \times \mathbb{G}_{m}}^{*}\left(\tilde{X}^{!} ; \mathbb{C}\right)
$$

Moreover, we have an isomorphism

$$
H_{T^{!} \times \mathbb{G}_{m}}^{*}\left(\tilde{X}^{!} ; \mathbb{C}\right) \cong H_{\operatorname{Aut}\left(\tilde{X}^{!}\right) \times \mathbb{G}_{m}}^{*}\left(\tilde{X}^{!} ; \mathbb{C}\right) \otimes_{H_{\mathrm{Aut}\left(\tilde{X}^{!}\right)}^{*}(p t)} H_{T^{!}}^{*}(p t)
$$

This isomorphism is compatible with quantum multiplication by divisors, thus

$$
Q_{\mathrm{reg}}^{!} \cong\left(Q_{\mathrm{reg}}^{!}\right)^{W} \otimes_{H_{\mathrm{Aut}\left(\tilde{X}^{!}\right)}^{*}}(p t) H_{T^{!}}^{*}(p t)
$$

as $E_{\mathrm{reg}}^{!}$-modules. Comparing (7) and (8) shows that Conjecture 5.5 implies Conjecture 5.1.

\subsection{Beyond the regular locus}

In Section 3, we defined a module $M$ over $R$, and then localized to obtain a module $M_{\text {reg }}$ over $R_{\text {reg. }}$. In Section 4 , however, we did not define a module $Q$ over $E$ that localizes to 
the module $Q_{\text {reg }}$ over $E_{\text {reg }}$. To this end, we now define $Q$ to be the $E$-submodule of $Q_{\text {reg }}$ generated by the vector subspace

$$
1 \otimes H_{T \times \mathbb{G}_{m}}^{*}(\tilde{X} ; \mathbb{C}) \subset F_{\text {reg }} \otimes H_{T \times \mathbb{G}_{m}}^{*}(\tilde{X} ; \mathbb{C})=Q_{\text {reg }}
$$

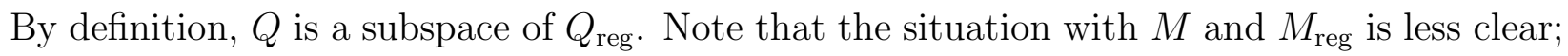
there is a natural map from $M$ to $M_{\text {reg }}$, but this map could a priori fail to be injective if $M$ has nontrivial $S$-torsion. Nonetheless, for any particular symplectic dual pair, it is natural to ask the following question.

Question 5.6. Do we have an isomorphism $M \cong Q^{!}$of graded modules over $R \cong E^{!}$?

In the hypertoric case, Question 5.6 is equivalent to the question of whether or not $M$ is torsion-free as a module over $S$. We believe that the answer is yes, and this problem will be addressed in a future work. In the case of the Springer resolution, we believe that the answer is yes in type $\mathrm{A}$ and no in other types. It is interesting to note that the Springer resolution can be realized as a quiver variety only in type A, so it is possible that the answer to Question 5.6 is yes for all dual pairs arising from the Higgs/Coulomb construction associated with a linear representation of a group.

Remark 5.7. In a case where the answer to Question 5.6 is yes, we obtain an isomorphism $\left(Q_{1}^{!}\right)^{\hbar=1} \cong M_{1}^{\hbar=1} \cong H H_{0}\left(\mathscr{A}^{\hbar=1}\right)$ by Proposition 3.12. The second and third authors have conjectured that $\left(Q_{1}^{!}\right)^{\hbar=1} \cong I H_{T^{!} \times \mathbb{G}_{m}}^{*}\left(X^{!} ; \mathbb{C}\right)^{\hbar=1}$ [MP15, Conjecture 2.5]. On the other hand, the third author has conjectured that $H H_{0}\left(\mathscr{A}^{\hbar=1}\right)$ is isomorphic to $I H_{T^{!} \times \mathbb{G}_{m}}^{*}\left(X^{!} ; \mathbb{C}\right)^{\hbar=1}[$ Pro14, Conjecture 3.6]. Thus a positive answer to Question [5.6 would make these two conjectures equivalent to each other.

\section{The hypertoric case}

The purpose of this section is to prove Conjecture 5.1 for hypertoric varieties. We begin with a review of quantization, quantum cohomology, and symplectic duality in the hypertoric setting.

\subsection{Hypertoric varieties}

Fix an algebraic torus $T$, a positive integer $n$, and an $n$-tuple $\gamma \in\left(\mathfrak{t}_{\mathbb{Z}}\right)^{n}$ satisfying the following conditions:

(1) For all $i, \gamma_{i}$ is nonzero, primitive, and contained in the span of $\left\{\gamma_{j} \mid j \neq i\right\}$. 
(2) The semigroup $\mathbb{N}\left\{\gamma_{1}, \ldots, \gamma_{n}\right\}$ spanned by $\gamma$ is equal to $\mathfrak{t}_{\mathbb{Z}}$.

(3) For any subset $S \subset[n]$, if $\left\{\gamma_{i} \mid i \in S\right\}$ is a $\mathbb{C}$-basis for $\mathfrak{t}$, then it is also a $\mathbb{Z}$-basis for $\mathfrak{t}_{\mathbb{Z}}$. These cocharacters define a surjective map from $\mathbb{G}_{m}^{n}$ to $T$, and we let $K$ denote the kernel of this map. Consider the action of $\mathbb{G}_{m}^{n}$ on $\mathbb{C}^{n}$ by coordinate-wise scalar multiplication. This induces a symplectic action on $T^{*} \mathbb{C}^{n}$, and the subtorus $K \subset \mathbb{G}_{m}^{n}$ acts with moment map $\mu: T^{*} \mathbb{C}^{n} \rightarrow \mathfrak{k}^{*}$, where $\mu(q, p)$ is the projection of $\left(q_{1} p_{1}, \ldots, q_{n} p_{n}\right) \in \mathbb{C}^{n} \cong$ Lie $\left(\mathbb{G}_{m}^{n}\right)^{*}$ to $\mathfrak{k}^{*}$. Let

$$
X:=\mu^{-1}(0) / / 0 K=\operatorname{Spec} \mathcal{O}\left(\mu^{-1}(0)\right)^{K}
$$

denote the the affine symplectic quotient of $T^{*} \mathbb{C}^{n}$ by $K$. The inverse scaling action of $\mathbb{G}_{m}$ on $T^{*} \mathbb{C}^{n}$ induces an action on $X$ for which $\mathcal{O}(X)$ is non-negatively graded with only constants in degree 0 and the natural Poisson bracket has weight -2 . The fact that the degree 1 part of $\mathcal{O}(X)$ is trivial follows from the last part of item (1). Fix a character $\theta \in \mathfrak{k}_{\mathbb{Z}}^{*}$ and consider the GIT quotient

$$
\tilde{X}:=\mu^{-1}(0) / /{ }_{\theta} K=\operatorname{Proj}\left(\mathcal{O}\left(\mu^{-1}(0)\right) \otimes \mathbb{C}[t]\right)^{K}
$$

where Proj is taken with respect to the grading given by powers of $t$ and $K$ acts on $t$ with weight $\theta$. We will assume that $\theta$ is chosen generically; this, along with item (3), implies that $\tilde{X}$ is smooth, and therefore a symplectic resolution of $X$ [BD00, Theorem 3.2]. The symplectic action of $\mathbb{G}_{m}^{n}$ on $T^{*} \mathbb{C}^{n}$ descends to an action of $T$ on $X$ and $\tilde{X}$ commuting with the conical action of $\mathbb{G}_{m}$. This realizes $T$ as a maximal torus of $\operatorname{Aut}(X)$. We have canonical isomorphisms

$$
\mathscr{X} \cong T^{*} \mathbb{C}^{n} / / 0 K=\operatorname{Spec} \mathcal{O}\left(T^{*} \mathbb{C}^{n}\right)^{K} \quad \text { and } \quad \tilde{\mathscr{X}} \cong T^{*} \mathbb{C}^{n} / /{ }_{\theta} K
$$

\subsection{The hypertoric enveloping algebra}

Let $D$ be the $\mathbb{C}[\hbar]$-algebra generated by degree 1 elements $z_{1}, \ldots, z_{n}$ and $w_{1}, \ldots, w_{n}$ with $\left[z_{i}, z_{j}\right]=0=\left[w_{i}, w_{j}\right]$ and $\left[w_{i}, z_{j}\right]=\delta_{i j} \hbar$. Then the hypertoric enveloping algebra $\mathscr{A}:=D^{K}$ is the canonical quantization of $\mathscr{X}$. Let $a_{i}:=z_{i} w_{i}$, so that $\mathscr{A}_{0}=\mathbb{C}\left[a_{1}, \ldots, a_{n}, \hbar\right]$. Note that, in the hypertoric case, the canonical ring homomorphism Sym $\mathscr{A}_{0}^{2} \rightarrow \mathscr{A}_{0}$ is an isomorphism.

For all $\lambda \in \mathfrak{t}_{\mathbb{Z}}^{*}$, let $\lambda_{i}:=\left\langle\lambda, \gamma_{i}\right\rangle \in \mathbb{Z}$. The map $\mathfrak{t}_{\mathbb{Z}}^{*} \hookrightarrow \mathbb{Z}^{n}$ taking $\lambda$ to $\left(\lambda_{1}, \ldots, \lambda_{n}\right)$ coincides with the inclusion of character lattices induced by the surjection from $\mathbb{G}_{m}^{n}$ to $T$. For all $\lambda$, there is a unique expression of the form $\lambda=\lambda_{+}-\lambda_{-}$where $\lambda_{+}, \lambda_{-} \in \mathbb{N}^{n}$ have disjoint

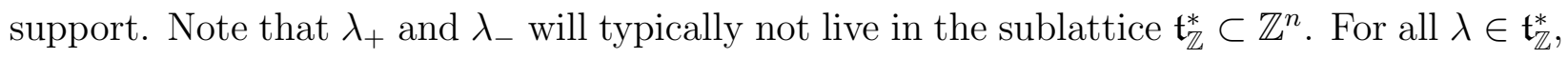
let $m^{\lambda}:=z^{\lambda_{+}} w^{\lambda_{-}} \in \mathscr{A}_{\lambda}$. Then we have $\mathscr{A}_{\lambda}=\mathscr{A}_{0} m^{\lambda}$. 
For any $k \in \mathbb{Z}$, let

$$
\left[a_{i}\right]^{k}:=\left\{\begin{array}{l}
1 \text { if } k=0 \\
a_{i}\left(a_{i}-\hbar\right) \cdots\left(a_{i}-(k-1) \hbar\right) \text { if } k>0 \\
\left(a_{i}+\hbar\right)\left(a_{i}+2 \hbar\right) \cdots\left(a_{i}-k \hbar\right) \text { if } k<0
\end{array}\right.
$$

Let $\lambda_{i}:=\left\langle\lambda, \gamma_{i}\right\rangle$ be the $i^{\text {th }}$ coordinate of $\lambda \in \mathbb{Z}^{n}$. Then we have the following description of the product structure of $\mathscr{A}[$ Hil16, Section 3.2]:

$$
\left[a_{i}, m^{\lambda}\right]=\lambda_{i} \hbar m^{\lambda} \quad \text { and } \quad m^{\lambda} m^{\mu}=\left(\prod_{\substack{\lambda_{i} \mu_{i}<0 \\ \lambda_{i}|\leq| \mu_{i} \mid}}\left[a_{i}\right]^{\lambda_{i}}\right) m^{\lambda+\mu}\left(\prod_{\substack{\lambda_{i} \mu_{i}<0 \\\left|\lambda_{i}\right|>\left|\mu_{i}\right|}}\left[a_{i}\right]^{-\mu_{i}}\right) .
$$

\subsection{Cocircuits and equivariant roots}

A nonzero primitive element of $\mathfrak{t}_{\mathbb{Z}}^{*}$ with minimal support is called a cocircuit. Condition (3) in Section 6.1 implies that, for any cocircuit $\lambda$, we have $\lambda_{i} \in\{-1,0,1\}$ for all $i$.

Lemma 6.1. For all $\lambda \in \mathfrak{t}_{\mathbb{Z}}^{*}$, there exist cocircuits $\mu^{1}, \ldots, \mu^{m}$, each supported on a subset of $\operatorname{Supp}(\lambda)$, such that

$$
\lambda=\mu^{1}+\cdots+\mu^{m}
$$

Proof. We will proceed by induction on the support of $\lambda$. If $\lambda=0$, we are done. If not, choose a cocircuit $\mu$ such that $\operatorname{Supp}(\mu) \subset \operatorname{Supp}(\lambda)$, and choose an element $i \in \operatorname{Supp}(\mu)$. Then $\lambda-\left(\lambda_{i} / \mu_{i}\right) \mu$ has support contained in $\operatorname{Supp}(\lambda) \backslash\{i\}$. Since $\mu$ is a cocircuit, $\mu_{i}= \pm 1$, so $\lambda_{i} / \mu_{i}$ is an integer.

We call Equation (11) cancellation free if $\mu_{i}^{k} \mu_{i}^{l} \geq 0$ for all $i, k, l$.

Lemma 6.2. For any $\lambda \in \mathfrak{t}_{\mathbb{Z}}^{*}$ we may choose $\mu^{1}, \ldots, \mu^{m}$ as in Lemma 6.1 such that Equation (11) is cancellation free.

Proof. We will again proceed by induction on the support of $\lambda$. If the support of $\lambda$ is minimal, then $\lambda$ is a multiple of a cocircuit, and we are done. Otherwise, choose cocircuits $\mu^{1}, \ldots, \mu^{m}$ such that $\lambda=\mu^{1}+\cdots+\mu^{m}$ and $\operatorname{Supp}\left(\mu^{k}\right) \subset \operatorname{Supp}(\lambda)$ for all $k$, which we can do by Lemma 6.1. Since each $\mu^{k}$ is a cocircuit, we have $\mu_{i}^{k} \mu_{i}^{l} \in\{-1,0,1\}$ for all $i, k, l$.

Let $i$ be the first coordinate such that there exist $k, l$ with $\mu_{i}^{k} \mu_{i}^{l}=-1$. Let $d$ be the minimum of $\left|\left\{k \mid \mu_{i}^{k}=1\right\}\right|$ and $\left|\left\{k \mid \mu_{i}^{k}=-1\right\}\right|$; we call this the degree of cancellation in the $i^{\text {th }}$ coordinate. We will produce a new expression for $\lambda$ that has a degree of cancellation of $d-1$ in the $i^{\text {th }}$ coordinate and still has no cancellation in the $j^{\text {th }}$ coordinate for $j<i$. By 
a second induction, this time on the index $i$, this will imply that we can obtain a cancellation free expression for $\lambda$.

Choose $k$ and $l$ such that $\mu_{i}^{k} \mu_{i}^{l}=-1$. This means that $\mu_{i}^{k}+\mu_{i}^{l}=0$, so we have $\operatorname{Supp}\left(\mu^{k}+\right.$ $\left.\mu^{l}\right) \subset \operatorname{Supp}(\lambda) \backslash\{i\}$. By our (first) inductive hypothesis, there exist cocircuits $\nu^{1}, \ldots, \nu^{s}$ such that $\operatorname{Supp}\left(\nu^{t}\right) \subset \operatorname{Supp}\left(\mu^{k}+\mu^{l}\right)$ for all $1 \leq t \leq s, \mu^{k}+\mu^{l}=\nu^{1}+\cdots+\nu^{s}$, and this sum is cancellation free. Then we have

$$
\lambda=\mu^{1}+\cdots+\widehat{\mu^{k}}+\cdots+\widehat{\mu^{l}}+\cdots+\mu^{m}+\nu^{1}+\cdots+\nu^{s}
$$

with the support of each of the cocircuits on the right-hand side contained in the support of $\lambda$, and with a degree of cancellation of $d-1$ in the $i^{\text {th }}$ coordinate. Thus it remains only to show that this expression has no cancellation in the $j^{\text {th }}$ coordinate when $j<i$.

Assume that there is cancellation in the $j^{\text {th }}$ coordinate for some $j<i$; this means that we have indices $q$ and $p$ such that $\mu_{j}^{q} \nu_{j}^{p}=-1$. Assume further that $\mu_{j}^{q}=1$ and $\nu_{j}^{p}=-1$ (the opposite case is identical). Since $\mu_{j}^{q}=1$ and the sum $\mu^{1}+\cdots+\mu^{m}$ has no cancellation in the $j^{\text {th }}$ coordinate, we have $\mu_{j}^{k}, \mu_{j}^{l} \in\{0,1\}$, and in particular the $j^{\text {th }}$ coordinate of $\mu^{k}+\mu^{l}=\nu^{1}+\cdots+\nu^{s}$ is non-negative. Since the sum on the right is cancellation-free, this implies that $\nu_{j}^{p} \in\{0,1\}$, which contradicts our assumption.

Proposition 6.3. The equivariant roots of $X$ are precisely the cocircuits.

Proof. If $\lambda$ is a cocircuit, it is clear that $m^{\lambda} \in \mathscr{A}^{+} \backslash\left(\mathscr{A}^{+}\right)^{2}$, so $\lambda$ is an equivariant root. By definition, 0 is not an equivariant root. Now suppose that $\lambda \neq 0$ is not a cocircuit. By Lemma 6.2, we may write $\lambda=\mu+\nu$, where $\mu, \nu \in \mathfrak{t}_{\mathbb{Z}}^{*}$ and $\mu_{i} \nu_{i} \geq 0$ for all $i$. Then Equation (10) tells us that $m^{\lambda}=m^{\mu} m^{\nu}$, and therefore $\mathscr{A}_{\lambda}=\mathscr{A}_{0} m^{\lambda} \subset\left(\mathscr{A}^{+}\right)^{2}$, so $\lambda$ is not an equivariant root.

Fix an element $\xi \in \mathfrak{t}_{\mathbb{Z}}$ such that $\langle\xi, \lambda\rangle \neq 0$ for every cocircuit $\lambda$. We call a cocircuit positive if $\langle\xi, \lambda\rangle>0$. By Proposition 6.3, equivariant roots are the same as cocircuits, so $\Sigma_{+}$is equal to the set of positive cocircuits.

Example 6.4. It is tempting to think that every element of $\mathbb{N} \Sigma_{+}$can be written as a cancellation-free sum of positive cocircuits. Unfortunately, this is not the case, as we illustrate here. Let $T:=\mathbb{G}_{m}^{3}$. Let $\left\{e_{1}, e_{2}, e_{3}\right\}$ be the coordinate basis for the character lattice $\mathfrak{t}_{\mathbb{Z}}^{*}$, and let $\left\{x_{1}, x_{2}, x_{3}\right\}$ be the dual basis for the cocharacter lattice $\mathfrak{t}_{\mathbb{Z}}$. Let $\gamma_{1}:=x_{1}, \gamma_{2}:=x_{2}$, $\gamma_{3}:=x_{3}, \gamma_{4}:=x_{1}-x_{3}$, and $\gamma_{5}:=x_{2}-x_{3}$. Let $\xi:=x_{1}-3 x_{2}+x_{3}$. Then we have $\Sigma_{+}=\left\{e_{1},-e_{2}, e_{3},-e_{2}-e_{3}, e_{1}+e_{3},-e_{1}-e_{2}-e_{3}\right\}$. Let $\lambda:=-e_{1}-e_{2}$. We can write $\lambda$ as the sum of two positive cocircuits $\left(e_{3}\right.$ and $\left.-e_{1}-e_{2}-e_{3}\right)$ or as the cancellation-free sum of two cocircuits $\left(-e_{1}\right.$ and $\left.-e_{2}\right)$, but not as the cancellation-free sum of two positive cocircuits. 


\subsection{The R-module M}

Let $S:=\mathbb{C}\left\{q^{\lambda} \mid \lambda \in \mathbb{N} \Sigma_{+}\right\}$as in Section 3.2. Since Sym $\mathscr{A}_{0}^{2} \cong \mathscr{A}_{0}$, we have

$$
R:=S \otimes \operatorname{Sym} \mathscr{A}_{0}^{2} \cong S \otimes \mathscr{A}_{0},
$$

and the left $R$-module $S \otimes \mathscr{A}_{0}$ is simply the left regular module. Let $R_{T}:=\mathcal{O}(T) \otimes_{S} R$. Since $R$ is free as an $S$-module, the natural map from $R$ to $R_{T}$ is an inclusion, thus we can freely work inside of $R_{T}$ when doing calculations in $R$.

For any $f(a) \in \mathscr{A}_{0}$ and $\lambda \in \mathfrak{t}_{\mathbb{Z}}^{*}$, let

$$
f_{\lambda}(a):=f\left(a_{1}+\lambda_{1} \hbar, \ldots, a_{n}+\lambda_{n} \hbar\right)
$$

so that we have

$$
f(a) m^{\lambda}=m^{\lambda} f_{\lambda}(a) \in \mathscr{A} \quad \text { and } \quad f(a) q^{\lambda}=q^{\lambda} f_{\lambda}(a) \in R_{T}
$$

For any $\lambda \in \mathfrak{t}_{\mathbb{Z}}^{*}$, let

$$
[a]^{\lambda}:=\prod_{i}\left[a_{i}\right]^{\lambda_{i}}=m^{\lambda} m^{-\lambda} \in \mathscr{A}_{0}
$$

We observe that, for all $\lambda \in \mathfrak{t}_{\mathbb{Z}}^{*}$, we have $[a]_{\lambda}^{\lambda}=[a]^{-\lambda}$.

By definition, $M$ is the left $R$-module $R / J$, where $J$ is the left ideal generated by elements of the form

$$
\begin{aligned}
f(a) m^{\lambda} g(a) m^{-\lambda}-q^{\lambda} g(a) m^{-\lambda} f(a) m^{\lambda} & =f(a) g_{-\lambda}(a) m^{\lambda} m^{-\lambda}-q^{\lambda} g(a) f_{\lambda}(a) m^{-\lambda} m^{\lambda} \\
& =f(a) g_{-\lambda}(a)[a]^{\lambda}-f(a) g_{-\lambda}(a) q^{\lambda}[a]^{-\lambda} \\
& =f(a) g_{-\lambda}(a)[a]^{\lambda}\left(1-q^{\lambda}\right)
\end{aligned}
$$

for $f(a), g(a) \in \mathscr{A}_{0}$ and $\lambda \in \mathbb{N} \Sigma_{+}$. Therefore, if we define

$$
r(\lambda):=[a]^{\lambda}\left(1-q^{\lambda}\right)
$$

then

$$
J=R \cdot\left\{r(\lambda) \mid \lambda \in \mathbb{N} \Sigma_{+}\right\}
$$

The rest of this section will be devoted to proving Proposition 6.8, which says that $J$ is in fact generated by those classes $r(\lambda)$ for $\lambda$ a positive cocircuit (rather than a sum of positive cocircuits). The following three lemmas are completely straightforward, so we omit their proofs.

Lemma 6.5. For any $\lambda \in \mathfrak{t}_{\mathbb{Z}}^{*}, r(\lambda)=-q^{\lambda} r(-\lambda) \in R_{T}$. 
Lemma 6.6. If $\lambda=\mu+\nu$, then $r(\lambda)=q^{\nu}[a]_{\nu}^{\lambda}\left(1-q^{\mu}\right)+[a]^{\lambda}\left(1-q^{\nu}\right) \in R_{T}$.

Lemma 6.7. If $\mu, \nu \in \mathfrak{t}_{\mathbb{Z}}^{*}$ and $\mu_{i} \nu_{i} \geq 0$ for all $i$, then there exist $f(a), g(a) \in \mathscr{A}_{0}$ such that

$$
[a]_{\nu}^{\mu+\nu}=f(a)[a]^{\mu} \quad \text { and } \quad[a]^{\mu+\nu}=g(a)[a]^{\nu} .
$$

Proposition 6.8. We have

$$
J=R \cdot\left\{r(\lambda) \mid \lambda \in \Sigma_{+}\right\} .
$$

Proof. Let $J^{\prime}:=R \cdot\left\{r(\lambda) \mid \lambda \in \Sigma_{+}\right\}$. We need to show that, if $\lambda \in \mathbb{N} \Sigma_{+}$, then $r(\lambda) \in J^{\prime}$. By Lemma 6.2, we can choose cocircuits $\mu^{1}, \ldots, \mu^{m}$ (not necessarily positive) such that $\lambda=\mu^{1}+\cdots+\mu^{m}$ and this sum is cancellation-free. We will prove that $r(\lambda) \in J^{\prime}$ by induction on $m$. The base case $m=0$ follows from the fact that $r(0)=0$.

Let us first assume that $\mu^{i} \in \Sigma_{+}$for all $i$. Let $\mu=\mu^{m}$ and $\nu=\mu^{1}+\cdots+\mu^{m-1}$. By Lemma 6.6, we have

$$
r(\lambda)=q^{\nu}[a]_{\nu}^{\lambda}\left(1-q^{\mu}\right)+[a]^{\lambda}\left(1-q^{\nu}\right) .
$$

By Lemma 6.7, there are elements $f(a), g(a) \in \mathscr{A}_{0}$ such that

$$
\begin{aligned}
r(\lambda) & =q^{\nu} f(a)[a]^{\mu}\left(1-q^{\mu}\right)+g(a)[a]^{\nu}\left(1-q^{\nu}\right) \\
& =q^{\nu} f(a) r(\mu)+g(a) r(\nu) .
\end{aligned}
$$

Our inductive hypothesis tells us that $r(\mu) \in J^{\prime}$, so we are done.

Next, assume that there is at least one index $i$ for which $-\mu_{i} \in \Sigma_{+}$. After reordering, we may assume that $i=m$. Once again, let $\mu=\mu^{m}$ and $\nu=\mu^{1}+\cdots+\mu^{m-1}$. By the same reasoning as above, there are elements $f(a), g(a) \in \mathscr{A}_{0}$ such that

$$
r(\lambda)=q^{\nu} f(a) r(\mu)+g(a) r(\nu)
$$

By Lemma 6.5, we have $r(\mu)=-q^{\mu} r(-\mu)$, so

$$
\begin{aligned}
r(\lambda) & =-q^{\nu} f(a) q^{\mu} r(-\mu)+g(a) r(\nu) \\
& =-q^{\nu+\mu} f_{\mu}(a) r(-\mu)+g(a) r(\nu) \\
& =-q^{\lambda} f_{\mu}(a) r(-\mu)+g(a) r(\nu) .
\end{aligned}
$$

Since $-\mu \in \Sigma_{+}, r(-\mu) \in J^{\prime}$. Since $\nu=\lambda+(-\mu) \in \mathbb{N} \Sigma_{+}$and $\nu$ is a cancellation-free sum of $m-1$ cocircuits, our inductive hypothesis tells us that $r(\nu) \in J^{\prime}$. Thus $r(\lambda) \in J^{\prime}$, as desired.

Remark 6.9. If we knew that $\lambda \in \mathbb{N} \Sigma_{+}$could be written as a cancellation-free sum of positive cocircuits, then the last paragraph of the proof of Proposition 6.8 would be unnecessary. 
However, this is not always the case, as we saw in Example 6.4. In that particular example, we have $r(\lambda)=-q^{\lambda} a_{2} a_{5} r\left(e_{1}\right)+\left(a_{1}+\hbar\right)\left(a_{4}+\hbar\right) r\left(-e_{2}\right)$.

\subsection{The $\mathrm{E}_{\mathrm{reg}}-$ module $\mathrm{Q}_{\mathrm{reg}}$}

Recall that

$$
E:=F \otimes \operatorname{Sym} H_{T \times \mathbb{G}_{m}}^{2}(\tilde{X} ; \mathbb{C})
$$

and $E_{\text {reg }}$ is the Ore localization of $E$ obtained by inverting $\left(1-q^{\alpha}\right)$ for all $\alpha \in \Delta_{+}$.

Proposition 6.10. The $E_{\mathrm{reg}}-$ module $Q_{\mathrm{reg}}$ is cyclic, generated by the class $1 \otimes 1 \in Q_{\mathrm{reg}}$.

Proof. Given a $k$-tuple $u=\left(u_{1}, \ldots, u_{k}\right) \in H_{T \times \mathbb{G}_{m}}^{\bullet}(\tilde{X} ; \mathbb{C})^{k}$, we define $u_{*} \in Q_{\text {reg }}$ to be the quantum product of the entries, and we define $u_{\cup} \in Q$ to be the tensor product of $1 \in F$ with the classical product of the entries. We call $u$ classical if $u_{*}=u_{\cup}$. It is easy to see that, if $u$ is a classical tuple, then $u_{1} u_{2} \cdots u_{k} \cdot 1 \otimes 1= \pm 1 \otimes u_{\cup}$ in $Q_{\text {reg. }}$ The cohomology ring $H_{T \times \mathbb{G}_{m}}^{\bullet}(\tilde{X} ; \mathbb{C})$ is spanned over $H_{T \times \mathbb{G}_{m}}^{\bullet}(p t ; \mathbb{C})$ by products of classical tuples of divisors [MP15, Corollary 3.3 and Lemma 3.4], therefore $Q_{\text {reg }}$ is generated by $1 \otimes 1$.

It follows from Proposition 6.10 that $Q_{\text {reg }}$ is isomorphic as an $E_{\text {reg }}$-module to the quotient of the regular module $E_{\text {reg }}$ by some left ideal, namely the annihilator of $1 \otimes 1$. Our goal is now to compute that ideal.

By definition, we have $K \subset \mathbb{G}_{m}^{n}$ and therefore $\mathfrak{k}_{\mathbb{Z}} \subset \mathbb{Z}^{n}$. For any $\alpha \in \mathfrak{k}_{\mathbb{Z}}$ and $i \leq n$, we define $\alpha_{i} \in \mathbb{Z}$ to be its $i^{\text {th }}$ coordinate. A nonzero primitive element of $\mathfrak{k}_{\mathbb{Z}}$ with minimal support is called a circuit. Each circuit pairs nontrivially with the element $\theta \in \mathfrak{k}_{\mathbb{Z}}^{*}$, and we call a circuit $\alpha$ positive if $\langle\alpha, \theta\rangle>0$.

Let $\chi_{1}, \ldots, \chi_{n}$ be the coordinate basis for $\mathbb{Z}^{n}=\operatorname{Hom}\left(\mathbb{G}_{m}^{n}, \mathbb{G}_{m}\right)$. The equivariant Kirwan map is an isomorphism from $\mathbb{Z}\left[\chi_{1}, \ldots, \chi_{n}, \hbar / 2\right]$ to $H_{T \times \mathbb{G}_{m}}^{2}(\tilde{X} ; \mathbb{Z})$, which takes a character of $\mathbb{G}_{m}^{n} \times \mathbb{G}_{m}$ to the $T \times \mathbb{G}_{m}$ equivariant chern class of the associated line bundle on the quotient. We let $u_{i}$ be the image of $\chi_{i}-\hbar / 2$ Setting $\hbar / 2$ equal to zero and passing to the quotient $\mathfrak{k}_{\mathbb{Z}}^{*}$ of $\mathbb{Z}^{n}$, we obtain the ordinary Kirwan map, which is an isomorphism from $\mathfrak{k}_{\mathbb{Z}}^{*}$ to $H^{2}(\tilde{X}, \mathbb{Z})$ taking $\theta$ to the first Chern class of an ample line bundle. The dual isomorphism $\mathfrak{k}_{\mathbb{Z}} \cong H_{2}(\tilde{X}, \mathbb{Z})$ takes the circuits bijectively to the Kähler roots of $\tilde{X}$ and the positive circuits to the set $\Delta_{+}$of positive Kähler roots [MS13, Theorem 4.2].

For each positive circuit $\alpha \in \Delta_{+}$, let

$$
s(\alpha):=[u]^{\alpha}\left(1-q^{\alpha}\right) \in E_{\mathrm{reg}},
$$

\footnotetext{
${ }^{4}$ The class $\chi_{i}-\hbar / 2$ is the weight of the normal bundle to the hyperplane $\left\{z_{i}=0\right\}$ in $T^{*} \mathbb{C}^{n}$, and $u_{i}$ is therefore represented by the Borel-Moore cycle given by the image of this hyperplane in $\tilde{X}$.
} 
where $[u]^{\alpha} \in \operatorname{Sym} H_{T \times \mathbb{G}_{m}}^{2}(\tilde{X} ; \mathbb{C})$ is defined in a manner analogous to the definition of $[a]^{\lambda}$ in Equations (9) and (12). Let

$$
I_{\mathrm{reg}}:=E_{\mathrm{reg}} \cdot\left\{s(\alpha) \mid \alpha \in \Delta_{+}\right\}
$$

We will prove that $I_{\text {reg }} \subset E_{\text {reg }}$ is equal to the annihilator of $1 \otimes 1 \in Q_{\text {reg. }}$.

Let $\bar{E}_{\text {reg }}:=E_{\text {reg }} \otimes_{\mathbb{C}[\hbar]} \mathbb{C} \cong F \otimes H_{T}^{2}(\tilde{X} ; \mathbb{C})$ be the algebra obtained from $E_{\text {reg }}$ by setting $\hbar=0$. Similarly, let $\bar{Q}_{\mathrm{reg}}:=\bar{E}_{\mathrm{reg}} \otimes_{E_{\mathrm{reg}}} Q_{\mathrm{reg}}$ and $\bar{I}_{\mathrm{reg}}:=\bar{E}_{\mathrm{reg}} \otimes_{E_{\mathrm{reg}}} I_{\mathrm{reg}}$. For any $e \in E_{\mathrm{reg}}$, let $\bar{e}$ denote its image in $\bar{E}_{\text {reg }}$.

Lemma 6.11. The ideal $\bar{I}_{\text {reg }} \subset \bar{E}_{\text {reg }}$ is equal to the annihilator of $1 \otimes 1 \in \bar{Q}_{\text {reg. }}$.

Proof. Since $\bar{E}_{\text {reg }}$ is commutative, we have

$$
\overline{s(\alpha)}=\left(1-q^{\alpha}\right) \overline{[u]^{\alpha}}=\left(1-q^{\alpha}\right) \prod_{i=1}^{n} u_{i}^{\left|\alpha_{i}\right|}
$$

for every positive cocircuit $\alpha$. Since $\left(1-q^{\alpha}\right)$ is invertible, this implies that $\bar{I}_{\text {reg }}$ is generated by the square-free monomials in $u$ corresponding to supports of circuits. This in turn is equal the kernel of the natural map $\operatorname{Sym}_{T}^{2}(\tilde{X} ; \mathbb{C}) \rightarrow H_{T}^{*}(\tilde{X} ; \mathbb{C})$ [Kon99, Theorem 2.4], which is by definition the annihilator of $1 \otimes 1$.

Proposition 6.12. The ideal $I_{\mathrm{reg}} \subset E_{\mathrm{reg}}$ is equal to the annihilator of $1 \otimes 1 \in Q_{\mathrm{reg}}$.

Proof. The fact that each $s(\alpha)$ annihilates $1 \otimes 1$ is proved in [MS13, Proposition 6.4] 5 , thus $I_{\text {reg }}$ is contained in the annihilator of $1 \otimes 1$. For the opposite inclusion, let $e \in E_{\text {reg }}$ be a class of degree $k$ that annihilates $1 \otimes 1$. We will prove by induction on $k$ that $e \in I_{\text {reg. This }}$ is trivial if $k=0$, in which case we must have $e=0$. For general $k$, we know that $\bar{e} \in \bar{E}_{\text {reg }}$ annihilates $1 \otimes 1 \in \bar{Q}_{\text {reg }}$, and therefore Lemma 6.11 tells us that $\bar{e} \in \bar{I}_{\text {reg. This means that }}$ there exists some $i \in I_{\text {reg }}$ of degree $k$ and $e^{\prime} \in E_{\text {reg }}$ of degree $k-2$ such that $e=i+\hbar e^{\prime}$. Then $\hbar e^{\prime}=e-i$ annihilates $1 \otimes 1$. Since $Q_{\text {reg }}$ is a free module over $\mathbb{C}[\hbar]$, this implies that $e^{\prime}$ annihilates $1 \otimes 1$. By our inductive hypothesis, $e^{\prime} \in I_{\text {reg }}$, therefore $e \in I_{\text {reg }}$.

\footnotetext{
${ }^{5}$ The notations of MS13 compare with ours as follows. The function $q^{S}$ in that paper corresponds to our function $q^{\alpha}$, where $\alpha$ is the root associated to $S$. This differs by a factor of the "theta characteristic" $(-1)^{|S|}$ from the function $q^{\beta_{S}}$ which also appears in MS13, but plays no role here. The quantum connection in MS13] is defined via the formula $\frac{d}{d u}+u \star$ rather than $\frac{d}{d u}-u \star$, as in this paper. Thus the operator $\mathcal{E}_{i}$ in MS13 is conjugate to the operator $u_{i}$ on $Q_{\text {reg }}$ in this paper via the automorphism of $H_{T \times \mathbb{G}_{m}}^{*}(\tilde{X} ; \mathbb{C})$ which multiplies an element of $H_{T \times \mathbb{G}_{m}}^{2 n}(\tilde{X} ; \mathbb{C})$ by $(-1)^{n}$. Finally, the variable $\hbar$ in [MS13] is a primitive character of the dilating torus, whereas for us it is the weight of the symplectic form.
} 


\subsection{Duality}

Recall from Section 6.1 that we began with the data of $\gamma \in\left(\mathfrak{t}_{\mathbb{Z}}\right)^{n}$ satisfying three conditions. This can be interpreted as a surjective map $\mathbb{Z}^{n} \rightarrow \mathfrak{t}_{\mathbb{Z}}$, and we thus obtain an exact sequence

$$
0 \rightarrow \mathfrak{k}_{\mathbb{Z}} \rightarrow \mathbb{Z}^{n} \rightarrow \mathfrak{t}_{\mathbb{Z}} \rightarrow 0
$$

By dualizing this sequence, we obtain an element $\gamma^{!} \in\left(\mathfrak{k}_{\mathbb{Z}}^{*}\right)^{n}$, satisfying the same three conditions, known as the Gale dual of $\gamma$. We then have $T^{!} \cong K^{*}$ and $K^{!} \cong T^{*}$. Let $X^{!}$ be the corresponding hypertoric variety, and choose a generic element $\theta^{!} \in \mathfrak{t}_{\mathbb{Z}}$ to obtain a symplectic resolution $\tilde{X}^{!} \rightarrow X^{!}$. We have

$$
\left(\mathfrak{t}^{!}\right)^{*} \cong \mathfrak{k} \cong H_{2}(\tilde{X} ; \mathbb{C}) \quad \text { and } \quad H^{2}\left(\tilde{X}^{!} ; \mathbb{C}\right) \cong\left(\mathfrak{k}^{!}\right)^{*} \cong \mathfrak{t}
$$

via the Kirwan maps for $\tilde{X}$ and $\tilde{X}^{!}$, and

$$
H_{T^{!} \times \mathbb{G}_{m}}^{2}\left(\tilde{X}^{!} ; \mathbb{C}\right)=\mathbb{C}\left\{u_{1}^{!}, \ldots, u_{n}^{!}, \hbar\right\} \cong \mathbb{C}\left\{a_{1}, \ldots, a_{n}, \hbar\right\}=\mathscr{A}_{0}^{2}
$$

where the isomorphism takes $u_{i}^{!}$to $a_{i}$ and $\hbar$ to $\hbar$. These isomorphisms are easily seen to be compatible with the maps in the equivariant and quantum exact sequences, thus the first item in Section 5 is satisfied. The isomorphism $\mathfrak{t}_{\mathbb{Z}}^{*} \cong \mathfrak{k}_{\mathbb{Z}}^{!}$induces a bijection between cocircuits for $\gamma$ and circuits for $\gamma^{!}$, and therefore between equivariant roots for $X$ and Kähler roots for $\tilde{X}^{!}$. If we choose $\theta^{!}=\xi$, then the positive equivariant roots match the positive Kähler roots, and the second item in Section 5 is satisfied. A more formal proof of symplectic duality between $X$ and $X^{!}$appears in [BLPW16] [Theorem 10.8].

Theorem 6.13. Conjecture 5.1 holds for hypertoric varieties.

Proof. Proposition 6.8 tells us that $M \cong R / R \cdot\left\{r(\lambda) \mid \lambda \in \Sigma_{+}\right\}$, and therefore that $M_{\text {reg }} \cong R_{\text {reg }} / R_{\text {reg }} \cdot\left\{r(\lambda) \mid \lambda \in \Sigma_{+}\right\}$. On the other hand, Proposition 6.12 tells us that $Q_{\text {reg }}^{!} \cong S_{\text {reg }}^{!} / S_{\text {reg }}^{!} \cdot\left\{s(\alpha) \mid \alpha \in \Delta_{+}\right\}$. We know that $S_{\text {reg }}^{!} \cong R_{\text {reg }}$ and that the isomorphism $\mathfrak{t}_{\mathbb{Z}}^{*} \cong \mathfrak{k}_{\mathbb{Z}}^{!}$takes $\Sigma_{+}$to $\Delta_{+}$, thus the theorem follows from the fact that $r(\lambda)$ and $s(\alpha)$ are defined by the same formula.

\section{The Springer resolution}

Our goal in this section to prove Conjecture 5.5 for the Springer resolution. 


\subsection{The algebraic Harish-Chandra map}

Let $G$ be a semisimple complex group, which we assume to be of adjoint type. Following the notation of Example 2.4, we let $X:=\mathcal{N}$ and

$$
\mathscr{A}:=U_{\hbar} \mathfrak{g} \otimes_{Z\left(U_{\hbar} \mathfrak{g}\right)}(\operatorname{Sym} \mathfrak{t} \otimes \mathbb{C}[\hbar]),
$$

which implies that

$$
\mathscr{A}_{0}^{2}=\mathfrak{t} \oplus \mathbb{C} \hbar \oplus \mathfrak{t} .
$$

In particular, the quantization exact sequence (2) naturally splits. The Namikawa Weyl group coincides with the usual Weyl group $W$ of $G$, and we have

$$
\mathscr{A}^{W} \cong U_{\hbar} \mathfrak{g} \quad \text { and } \quad\left(\operatorname{Sym} \mathscr{A}_{0}^{2}\right)^{W} \cong \operatorname{Sym} \mathfrak{t} \otimes \mathbb{C}[\hbar] \otimes(\operatorname{Sym} \mathfrak{t})^{W}
$$

We define the Weyl vector $\rho \in \frac{1}{2} \mathfrak{t}_{\mathbb{Z}}^{*}$ to be half the sum of the positive roots, and the dual Weyl vector $\xi \in \mathfrak{t}_{\mathbb{Z}}$ to be half the sum of the positive coroots. The equivariant roots $\Sigma \subset \mathfrak{t}^{*}$ of $X$ coincide with the roots in the usual Lie theory sense, and the positive equivariant roots $\Sigma_{+} \subset \Sigma$ (those that pair positively with $\xi$ ) coincide with the positive roots in the Lie theory sense.

For every element $\lambda \in \mathfrak{t}^{*}$, we have an evaluation map Sym $\mathfrak{t} \rightarrow \mathbb{C}$. We can apply the Rees construction to this map and obtain a graded $\mathbb{C}[\hbar]$-algebra map Sym $\mathfrak{t} \otimes \mathbb{C}[\hbar] \rightarrow \mathbb{C}[\hbar]$ which we denote by $y \mapsto y(\lambda)$. For any $\mathfrak{g}$-module $V$, we obtain a module $V_{\hbar}$ over $U_{\hbar} \mathfrak{g}$ by taking the Rees construction (with respect to the trivial filtration on $V$ ). If $V$ is indecomposable (for example a simple module or a Verma module), then every $a \in Z\left(U_{\hbar} \mathfrak{g}\right)$ acts on $V_{\hbar}$ by some scalar $a(V) \in \mathbb{C}[\hbar]$. The resulting map $a \mapsto a(V)$ is a graded $\mathbb{C}[\hbar]$-algebra map $Z\left(U_{\hbar} \mathfrak{g}\right) \rightarrow \mathbb{C}[\hbar]$. Let $y \mapsto y_{\rho}$ be the graded $\mathbb{C}[\hbar]$-algebra automorphism of Sym $\mathfrak{t} \otimes \mathbb{C}[\hbar]$ defined by putting $x_{\rho}=x-\langle\rho, x\rangle \hbar$ for all $x \in \mathfrak{t}$. In particular, for any $\lambda \in \mathfrak{t}^{*}$ and $y \in \operatorname{Sym} \mathfrak{t} \otimes \mathbb{C}[\hbar]$, we have $y_{\rho}(\lambda)=y(\lambda-\rho)$.

We will refer to a finite-dimensional irreducible $\mathfrak{g}$-module as a $\boldsymbol{G}$-irrep. Such modules are classified by dominant weights; for any dominant weight $\lambda \in \mathfrak{t}_{\mathbb{Z}}^{*}$, let $V(\lambda)$ be the $G$-irrep of highest weight $\lambda$. The algebraic Harish-Chandra map is the unique graded $\mathbb{C}[\hbar]$-algebra map

$$
\varphi: Z\left(U_{\hbar} \mathfrak{g}\right) \rightarrow \operatorname{Sym} \mathfrak{t} \otimes \mathbb{C}[\hbar]
$$

with the property that, for any dominant weight $\lambda$ and any $a \in Z\left(U_{\hbar} \mathfrak{g}\right), \varphi(a)(\lambda+\rho)=$ $a(V(\lambda))$. 
Recall from Section 3.4 that we have

$$
B\left(U_{\hbar} \mathfrak{g}\right):=\left(U_{\hbar} \mathfrak{g}\right)_{0} / \sum_{\langle\mu, \xi\rangle>0}\left\{a b \mid a \in\left(U_{\hbar} \mathfrak{g}\right)_{\mu}, b \in\left(U_{\hbar} \mathfrak{g}\right)_{-\mu}\right\}
$$

We have a natural map $\psi:$ Sym $\mathfrak{t} \otimes \mathbb{C}[\hbar] \rightarrow B\left(U_{\hbar} \mathfrak{g}\right)$ coming from the inclusion $\mathfrak{t} \rightarrow \mathfrak{g}$. This map and the algebraic Harish-Chandra map are close to being mutually inverse isomorphisms. More precisely, we have the following standard results, see for example [Hum78, Section 23.3].

Proposition 7.1. The maps $\varphi$ and $\psi$ have the following properties.

1. The map $\varphi$ is injective with image $(\operatorname{Sym} \mathfrak{t})^{W} \otimes \mathbb{C}[\hbar]$.

2. The map $\psi$ is an isomorphism.

3. For any element $a \in Z\left(U_{\hbar} \mathfrak{g}\right) \subset B\left(U_{\hbar} \mathfrak{g}\right)$, we have $\psi\left(\varphi(a)_{\rho}\right)=a$.

By Proposition 7.1(1), we may use the algebraic Harish-Chandra map $\varphi$ to endow $U_{\hbar} \mathfrak{g}$, $B\left(U_{\hbar} \mathfrak{g}\right)$, and other related objects with an action of $(\operatorname{Sym} \mathfrak{t})^{W} \otimes \mathbb{C}[\hbar]$.

Example 7.2. Consider $\mathfrak{g}=\mathfrak{s l}_{2}$. Then $Z\left(U_{\hbar} \mathfrak{g}\right)=\mathbb{C}[C, \hbar]$ where

$$
C:=2 E F+2 F E+H^{2}+\hbar^{2}=4 F E+H^{2}+2 \hbar H+\hbar^{2}=4 E F+H^{2}-2 \hbar H+\hbar^{2} .
$$

We have Sym $\mathfrak{t}=\mathbb{C}[H]$ and $W=S_{2}$ acts by negating $H$, so $(\operatorname{Sym} \mathfrak{t})^{W} \otimes \mathbb{C}[\hbar]=\mathbb{C}\left[H^{2}, \hbar\right]$. Identify $\mathfrak{t}_{\mathbb{Z}}^{*}$ with $\mathbb{Z}$ by sending $\rho$ to 1 . Then we have

$$
\hbar^{2}(n+1)^{2}=C(V(n))=\varphi(C)(n+1)
$$

which implies that $\varphi(C)=H^{2}$. In $B\left(U_{\hbar} \mathfrak{s l}_{2}\right)$, the element $4 E F$ goes to 0 , so the image of $C$ in $B\left(U_{\hbar} \mathfrak{s l}_{2}\right)$ is $(H-\hbar)^{2}$.

\subsection{Equivariant Hikita}

Let $G^{!}$be the Langlands dual of $G$. Let

$$
X^{!}:=\mathcal{N}^{!} \quad \text { and } \quad \tilde{X}^{!}:=T^{*}\left(G^{!} / B^{!}\right)
$$

Since $G$ was assumed to be of adjoint type, we see that $G^{!}$is simply-connected, so $G^{!}$will typically contain a finite centre and thus not be equal to the automorphism of $X$ ! However, this will not affect the equivariant cohomology (over $\mathbb{C}$ ), so we will work with $G^{\text {! }}$ rather than $\operatorname{Aut}\left(X^{!}\right)$in order to simplify our notation. 
For any $\lambda \in \mathfrak{t}_{\mathbb{Z}}=\left(\mathfrak{t}^{!}\right)_{\mathbb{Z}}^{*}$, let $L(\lambda):=G^{!} \times \mathbb{C}_{\lambda} / B^{!}$be the associated line bundle on $G^{!} / B^{!}$. This line bundle carries a unique $G^{!}$-equivariant structure and therefore also a canonical $T^{!}$-equivariant structure. We endow it with a $G^{!} \times \mathbb{G}_{m}$-equivariant structure by letting $\mathbb{G}_{m}$ act with weight $\langle 2 \rho, \lambda\rangle \in \mathbb{Z}$, and we let $\tilde{L}(\lambda)$ denote the pullback of $L(\lambda)$ to $\tilde{X}^{!}$. (This non-standard choice of $\mathbb{G}_{m}$-equivariant structure on the line bundle will be necessary later for the equivariant Hikita isomorphism.)

We obtain an isomorphism $\mathfrak{t}_{\mathbb{Z}}=\left(\mathfrak{t}^{!}\right)_{\mathbb{Z}}^{*} \cong H^{2}\left(\tilde{X}^{!} ; \mathbb{Z}\right)$ by sending $\lambda$ to the (non-equivariant) Chern class of $\tilde{L}(\lambda)$. Moreover, the map taking $\lambda$ to the $T^{!} \times \mathbb{G}_{m}$-equivariant first Chern class of $\tilde{L}(\lambda)$ provides a splitting of the cohomology exact sequence (6). In particular, we have a canonical isomorphism

$$
H_{T^{!} \times \mathbb{G}_{m}}^{2}\left(\tilde{X}^{!} ; \mathbb{C}\right) \cong H^{2}\left(\tilde{X}^{!} ; \mathbb{C}\right) \oplus H_{T^{!} \times \mathbb{G}_{m}}^{2}(p t ; \mathbb{C}) \cong \mathfrak{t} \oplus \mathbb{C} \hbar \oplus \mathfrak{t}
$$

Taking symmetric algebras and $W$-invariants, we also have

$$
\left(\operatorname{Sym} H_{T^{!} \times \mathbb{G}_{m}}^{2}\left(\tilde{X}^{!} ; \mathbb{C}\right)\right)^{W} \cong \operatorname{Sym} \mathfrak{t} \otimes \mathbb{C}[\hbar] \otimes(\operatorname{Sym} \mathfrak{t})^{W} .
$$

Proposition 7.3. Consider the graded $\mathbb{C}[\hbar]$-algebra homomorphism

$$
\widetilde{\psi}: \operatorname{Sym} \mathfrak{t} \otimes \mathbb{C}[\hbar] \rightarrow H_{G^{!} \times \mathbb{G}_{m}}^{*}\left(\tilde{X}^{!} ; \mathbb{C}\right)
$$

taking $\lambda \mathfrak{t}_{\mathbb{Z}}$ to the $G^{!} \times \mathbb{G}_{m}$-equivariant first Chern class of $\tilde{L}(\lambda)$.

1. This map is an isomorphism.

2. The inclusion

$$
\begin{aligned}
& (\operatorname{Sym} \mathfrak{t})^{W} \otimes \mathbb{C}[\hbar] \cong H_{G^{\prime} \times \mathbb{G}_{m}}^{*}(p t ; \mathbb{C}) \rightarrow H_{G^{\prime} \times \mathbb{G}_{m}}^{*}\left(\tilde{X}^{!} ; \mathbb{C}\right) \cong \operatorname{Sym} \mathfrak{t} \otimes \mathbb{C}[\hbar] \\
& \text { takes y } \in(\operatorname{Sym} \mathfrak{t})^{W} \otimes \mathbb{C}[\hbar] \text { to } y_{\rho} \in \operatorname{Sym} \mathfrak{t} \otimes \mathbb{C}[\hbar] .
\end{aligned}
$$

Proof. The first statement follows from the fact that the projection from $T^{*}\left(G^{!} / B^{!}\right)$to $G^{!} / B^{!}$ is a homotopy equivalence, and we have

$$
H_{G^{!} \times \mathbb{G}_{m}}^{*}\left(G^{!} / B^{!} ; \mathbb{C}\right) \cong H_{G^{!} \times B^{!} \times \mathbb{G}_{m}}^{*}\left(G^{!} ; \mathbb{C}\right) \cong H_{B^{!} \times \mathbb{G}_{m}}^{*}(p t ; \mathbb{C}) \cong \operatorname{Sym} \mathfrak{t} \otimes \mathbb{C}[\hbar]
$$

The second statement follows from the way in which we defined the action of $\mathbb{G}_{m}$ on $\tilde{L}(\lambda)$.

We now check that the $W$-invariant version of the equivariant Hikita conjecture (Remark 5.3) holds for the Springer resolution. 
Proposition 7.4. There is an isomorphism $B\left(U_{\hbar} \mathfrak{g}\right) \cong H_{G^{!} \times \mathbb{G}_{m}}^{*}\left(\tilde{X}^{!}\right)$of graded algebras over the ring $\operatorname{Sym} \mathfrak{t} \otimes \mathbb{C}[\hbar] \otimes(\operatorname{Sym} \mathfrak{t})^{W}$.

Proof. Propositions 7.1(2) and 7.3(1) tell us that both rings are isomorphic to Sym $\mathfrak{t} \otimes \mathbb{C}[\hbar]$. The action of Sym $\mathfrak{t}$ on both rings is the obvious one, but the action of $\mathbb{C}[\hbar] \otimes(\operatorname{Sym} \mathfrak{t})^{W}$ is not so obvious. Propositions 7.1(3) and 7.3(2) tell us that, for both algebras, an element $y \in(\operatorname{Sym} \mathfrak{t})^{W} \otimes \mathbb{C}[\hbar]$ is mapped to $y_{\rho} \in \operatorname{Sym} \mathfrak{t} \otimes \mathbb{C}[\hbar]$.

\subsection{Differential operators}

By Langlands duality we have $\mathfrak{t}_{\mathbb{Z}}^{*}=\mathfrak{t}_{\mathbb{Z}}^{!} \cong H_{2}\left(\tilde{X}^{!} ; \mathbb{Z}\right)$. By [BMO11, Theorem 1.1], under this identification, the positive Kähler roots $\Delta_{+}^{!} \subset H_{2}\left(\tilde{X}^{!} ; \mathbb{Z}\right)$ coincide with the positive roots of $G$. In particular, this means that $\Delta_{+}^{!}=\Sigma_{+}$and

$$
F_{\mathrm{reg}}^{!}=\mathbb{C}\left[q^{\alpha},\left(1-q^{\alpha}\right)^{-1} \mid \alpha \in \Sigma_{+}\right]=S_{\mathrm{reg}} .
$$

Let $D_{\hbar}\left(T_{\text {reg }}\right)$ be the Rees algebra of the ring of differential operators on $T_{\text {reg }}:=T \cap$ Spec $S_{\text {reg }}$, filtered by order. Applying the Rees construction to the action of differential operators on functions, we obtain an action of $D_{\hbar}\left(T_{\text {reg }}\right)$ on $\mathcal{O}\left(T_{\text {reg }}\right) \otimes \mathbb{C}[\hbar]$. Each element $x \in \mathfrak{t}$ gives rise to an invariant vector field on $T_{\text {reg }}$, which induces a map $\iota: \operatorname{Sym} \mathfrak{t} \otimes \mathbb{C}[\hbar] \rightarrow D_{\hbar}\left(T_{\text {reg }}\right)$, and we have

$$
\iota(y) \cdot q^{\lambda}=y(\lambda) q^{\lambda}
$$

for all $y \in \operatorname{Sym} \mathfrak{t} \otimes \mathbb{C}[\hbar]$ and $\lambda \in \mathfrak{t}_{\mathbb{Z}}^{*}$.

Let $D_{\text {reg }}$ be the $\mathbb{C}[\hbar]$-subalgebra of $D_{\hbar}\left(T_{\text {reg }}\right)$ generated by the images of $\iota$ and $S_{\text {reg }} \subset$ $\mathcal{O}\left(T_{\text {reg }}\right)$. We then have a graded vector space isomorphism $D_{\text {reg }} \cong S_{\text {reg }} \otimes \operatorname{Sym} \mathfrak{t} \otimes \mathbb{C}[\hbar]$. We can also regard $D_{\text {reg }}$ as a subalgebra of $R_{\text {reg }}^{W} \cong\left(E_{\text {reg }}^{!}\right)^{W}$, and we have graded algebra isomorphisms

$$
R_{\text {reg }}^{W} \cong D_{\text {reg }} \otimes(\operatorname{Sym} \mathfrak{t})^{W} \cong\left(E_{\text {reg }}^{!}\right)^{W} .
$$

\subsection{The geometric Harish-Chandra map}

We thank Sam Gunningham for help with the proof of the following lemma.

Lemma 7.5. Let $X$ and $Y$ be smooth affine varieties over $\mathbb{C}$ and let $X \rightarrow Y$ be a dominant morphism of relative dimension zero. Suppose that $d \in D(X)$ and $d \cdot f=0$ for all $f \in$ $\mathcal{O}(Y) \subset \mathcal{O}(X)$. Then $d=0$.

Proof. We will prove the lemma by induction on the order of $d$. When the order is zero, $d$ is multiplication by a function, so the result holds. Now suppose that the lemma holds for 
differential operators of order at most $k-1$, and let $d$ be a differential operator of order at most $k$ such that $d \cdot f=0$ for all $f \in \mathcal{O}(Y)$. For any $f \in \mathcal{O}(Y)$, the commutator $[d, f] \in D(X)$ is a differential operator of order at most $k-1$. For any $g \in \mathcal{O}(Y)$, we have

$$
[d, f](g)=d \cdot(f g)-f d \cdot g=0
$$

Thus our inductive hypothesis tells us that $[d, f]=0$.

The ring $D(X)$ acts faithfully on the function field $\mathcal{K}(X)$, and the above paragraph implies that the element $d \in D(X)$ acts $\mathcal{K}(Y)$-linearly, and thus can be regarded as an element of $D(\mathcal{K}(X) / \mathcal{K}(Y))$. By the smoothness assumption, $D(\mathcal{K}(X) / \mathcal{K}(Y))$ is generated by $\mathcal{K}(X)$ and $\mathcal{K}(Y)$-linear derivations of $\mathcal{K}(X)$. Since our map has relative dimension zero, $\mathcal{K}(Y) / \mathcal{K}(X)$ is an algebraic extension, which implies that there are no such derivations. Thus $d \in \mathcal{K}(X)$ and the result follows.

Corollary 7.6. If $d \in D_{\hbar}\left(T_{\mathrm{reg}}\right)$ and $d \cdot \chi_{V}=0$ for all $G$-irreps $V$, then $d=0$.

Proof. We apply the Lemma 7.5 to the map $T_{\text {reg }} \rightarrow T \rightarrow T / W$. Since the characters of irreducible representations form a basis for $\mathcal{O}(T / W)$, the result follows.

By Corollary 7.6 , there is a unique graded $\mathbb{C}[\hbar]$-algebra homomorphism

$$
\Phi: Z\left(U_{\hbar} \mathfrak{g}\right) \rightarrow D_{\hbar}\left(T_{\text {reg }}\right)
$$

with the property that, for any $G$-irrep $V$ and any $a \in Z\left(U_{\hbar} \mathfrak{g}\right)$, we have $\Phi(a)\left(\chi_{V}\right)=a(V) \chi_{V}$. We call this homomorphism the geometric Harish-Chandra map. Let

$$
\delta:=\prod_{\alpha \in \Delta_{+}}\left(q^{\alpha}-1\right)
$$

denote the Weyl denominator. The algebraic Harish-Chandra map $\varphi$ and the geometric Harish-Chandra map $\Phi$ are related by the following lemma.

Lemma 7.7. For any $a \in Z\left(U_{\hbar} \mathfrak{g}\right)$, we have

$$
\Phi(a)=\delta^{-1} \iota\left(\varphi(a)_{\rho}\right) \delta
$$

In particular, the image of $\Phi$ is contained in the subalgebra $D_{\mathrm{reg}} \subset D_{\hbar}\left(T_{\mathrm{reg}}\right)$.

Proof. We need to show that

$$
\delta^{-1} \iota\left(\varphi(a)_{\rho}\right) \delta \cdot \chi_{V(\lambda)}=a(V(\lambda)) \cdot \chi_{V(\lambda)}
$$


for every dominant weight $\lambda$. The Weyl character formula says that

$$
\chi_{V(\lambda)}=\sum_{w \in W}(-1)^{\ell(w)} \frac{q^{w(\lambda+\rho)+\rho}}{\delta}
$$

and therefore we need to show that

$$
\iota\left(\varphi(a)_{\rho}\right) \cdot \sum_{w \in W}(-1)^{\ell(w)} q^{w(\lambda+\rho)+\rho}=a(V(\lambda)) \cdot \sum_{w \in W}(-1)^{\ell(w)} q^{w(\lambda+\rho)+\rho} .
$$

We will prove the equality summand-by-summand. Equation (13) tells us that

$$
\iota\left(\varphi(a)_{\rho}\right) \cdot q^{w(\lambda+\rho)+\rho}=\varphi(a)_{\rho}(w(\lambda+\rho)+\rho) \cdot q^{w(\lambda+\rho)+\rho}=\varphi(a)(w(\lambda+\rho)) \cdot q^{w(\lambda+\rho)+\rho} .
$$

By Weyl invariance of $\varphi(a)$, this is equal to $\varphi(a)(\lambda+\rho) \cdot q^{w(\lambda+\rho)+\rho}$, which by the definition of $\varphi$ is equal to $a(V(\lambda)) \cdot q^{w(\lambda+\rho)+\rho}$. This concludes the proof.

\subsection{The D-module of traces}

This section is devoted to computing the $R_{\mathrm{reg}}^{W}$-module

$$
M_{\text {reg }}^{W}=S_{\text {reg }} \otimes\left(U_{\hbar} \mathfrak{g}\right)_{0} / \sum_{\lambda \in \mathbb{N} \Sigma_{+}} S_{\text {reg }}[\hbar] \cdot\left\{1 \otimes a b-q^{\lambda} \otimes b a \mid a \in\left(U_{\hbar} \mathfrak{g}\right)_{\lambda}, b \in\left(U_{\hbar} \mathfrak{g}\right)_{-\lambda}\right\}
$$

We begin by proving that, as a module over the subalgebra $D_{\text {reg }} \subset R_{\text {reg }}^{W}, M_{\text {reg }}^{W}$ is isomorphic to the regular module.

Theorem 7.8. The map $\sigma: D_{\mathrm{reg}} \rightarrow M_{\mathrm{reg}}^{W}$ taking $d \in D_{\mathrm{reg}}$ to $d \cdot(1 \otimes 1) \in M_{\mathrm{reg}}^{W}$ is an isomorphism of graded $D_{\text {reg-modules. }}$

Proof. We begin by showing that $\sigma$ is surjective, which we will prove by induction on degree. Assume that $\sigma$ is surjective in all degrees less than $k$, and let $a \in\left(U_{\hbar} \mathfrak{g}\right)_{0}^{k}$. Write $a=$ $h+\sum_{\alpha \in \Delta_{+}} E_{\alpha} b_{\alpha}$, where $h \in$ Sym $\mathfrak{t}$ has degree $k$ and $b_{\alpha} \in\left(U_{\hbar} \mathfrak{g}\right)_{-\alpha}^{k-2}$. It is clear that the image of $h$ in $M_{\text {reg }}^{W}$ lies in the image of $\sigma$, so it is enough to prove that the images of each $E_{\alpha} b_{\alpha}$ in $M_{\text {reg }}^{W}$ lie in the image of $\sigma$, as well.

We know that there exists an element $c \in\left(U_{\hbar} \mathfrak{g}\right)_{0}^{k-2}$ such that $\left[b_{\alpha}, E_{\alpha}\right]=\hbar c$. In $M_{\text {reg }}^{W}$, we have

$$
E_{\alpha} b_{\alpha}=q^{\alpha} b_{\alpha} E_{\alpha}=q^{\alpha}\left(E_{\alpha} b_{\alpha}+\left[b_{\alpha}, E_{\alpha}\right]\right)=q^{\alpha}\left(E_{\alpha} b_{\alpha}+\hbar c\right),
$$

and therefore

$$
E_{\alpha} b_{\alpha}=\frac{q^{\alpha}}{1-q^{\alpha}} \hbar c
$$


By our inductive hypothesis, $c$ lies in the image of $\sigma$, and thus so does $E_{\alpha} b_{\alpha}$. This proves surjectivity.

To show injectivity of $\sigma$, recall from Proposition 3.15 that, for any $G$-irrep $V$, the map $\operatorname{tr}_{V}: M_{\text {reg }}^{W} \rightarrow \mathcal{O}\left(T_{\text {reg }}\right) \otimes \mathbb{C}[\hbar]$ is a $D_{\text {reg }}$-module map. Suppose that $d \in D_{\text {reg }}$ and that $\sigma(d)=0$. Then for any $G$-irrep $V$, we have

$$
0=\operatorname{tr}_{V}(0)=\operatorname{tr}_{V}(\sigma(d))=\operatorname{tr}_{V}(d \cdot(1 \otimes 1))=d \cdot \operatorname{tr}_{V}(1 \otimes 1)=d \cdot \chi_{V}
$$

Then Lemma 7.6 tells us that $d=0$.

It remains only to determine how $(\operatorname{Sym} \mathfrak{t})^{W} \otimes \mathbb{C}[\hbar] \subset R_{\text {reg }}^{W}$ acts on $M_{\text {reg }}^{W}$.

Lemma 7.9. For all $a \in Z\left(U_{\hbar} \mathfrak{g}\right)$ and $d \in D_{\text {reg }} \cong M_{\text {reg }}^{W}$, we have $a \cdot d=\Phi(a) d$.

Proof. Let $\Phi^{\prime}: Z\left(U_{\hbar} \mathfrak{g}\right) \rightarrow D_{\text {reg }}$ denote the composition

$$
Z\left(U_{\hbar} \mathfrak{g}\right) \rightarrow\left(U_{\hbar} \mathfrak{g}\right)_{0} \rightarrow M_{\text {reg }}^{W} \stackrel{\sigma^{-1}}{\longrightarrow} D_{\text {reg }}
$$

We wish to show that $\Phi^{\prime}=\Phi$. Fix an element $a \in Z\left(U_{\hbar} \mathfrak{g}\right)$. For any $G$-irrep $V$, Proposition 3.15 implies that

$$
\Phi^{\prime}(a) \cdot \chi_{V}=\Phi^{\prime}(a) \cdot \operatorname{tr}_{V}(1)=\operatorname{tr}_{V}(a)=a(V) \chi_{V}=\Phi(a) \cdot \chi_{V},
$$

thus $\left(\Phi(a)-\Phi^{\prime}(a)\right) \cdot \chi_{V}=0$. By Lemma 7.6, we conclude that $\Phi^{\prime}(a)=\Phi(a)$.

Lemmas 7.7 and 7.9 combine to give us the following result.

Proposition 7.10. For all $y \in(\operatorname{Sym} \mathfrak{t})^{W} \otimes \mathbb{C}[\hbar]$ and $d \in D_{\text {reg }} \cong M_{\text {reg }}^{W}$, we have

$$
y \cdot d=\delta^{-1} \iota\left(y_{\rho}\right) \delta d
$$

\subsection{The quantum D-module}

Consider the graded $D_{\text {reg }}$-module homomorphism $\Psi: D_{\text {reg }} \rightarrow\left(Q_{\text {reg }}^{!}\right)^{W}$ taking $d \in D_{\text {reg }}$ to $d \cdot(1 \otimes 1)$.

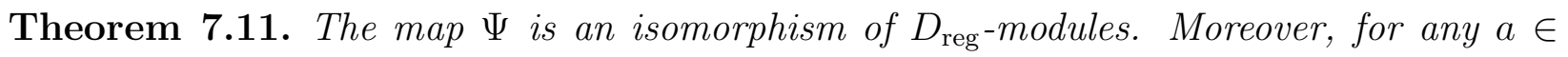
$(\operatorname{Sym} \mathfrak{t})^{W} \cong H_{G^{\prime}}^{*}(p t ; \mathbb{C})$, the image of a in $\left(Q_{\mathrm{reg}}^{!}\right)^{W}$ under the natural inclusion of the equivariant cohomology of a point into the equivariant cohomology of $T^{*}\left(G^{!} / B^{!}\right)$is equal to $\Psi\left(\delta^{-1} \iota\left(a_{\rho}\right) \delta\right)$.

Before proving this result, we recall the results of Braverman-Maulik-Okounkov [BMO11] describing the quantum connection of $T^{*}\left(G^{!} / B^{!}\right)$. We then relate this connection to the AKZ 
connection studied by Cherednick and Matsuo, and use this relationship to prove certain differential relations are satisfied in $\left(Q_{\mathrm{reg}}^{!}\right)^{W}$. With these relations in hand, we will conclude by proving Theorem 7.11 .

In order to cite the results of [BMO11], it will be convenient to use the more standard shifted isomorphism Sym $\mathfrak{t} \otimes \mathbb{C}[\hbar] \cong H_{G^{!} \times \mathbb{G}_{m}}^{*}\left(T^{*}\left(G^{!} / B^{!}\right) ; \mathbb{C}\right)$ defined by $\lambda \mapsto \widetilde{\psi}(\lambda)-\hbar\langle\rho, \lambda\rangle$ (for $\lambda \in \mathfrak{t}_{\mathbb{Z}}$ ). This isomorphism induces an isomorphism of graded vector spaces

$$
\left(Q_{\mathrm{reg}}^{!}\right)^{W}:=S_{\mathrm{reg}} \otimes H_{G^{!} \times \mathbb{G}_{m}}^{*}\left(T^{*}\left(G^{!} / B^{!}\right) ; \mathbb{C}\right) \cong S_{\mathrm{reg}} \otimes \operatorname{Sym} \mathfrak{t} \otimes \mathbb{C}[\hbar] .
$$

We will now describe the induced $\left(E_{\mathrm{reg}}^{!}\right)^{W}$-module structure on the right-hand space. Recall from Section 7.3 that $\left(E_{\mathrm{reg}}^{!}\right)^{W} \cong D_{\text {reg }} \otimes(\operatorname{Sym} \mathfrak{t})^{W}$, and that $D_{\text {reg }}$ is generated over $\mathbb{C}[\hbar]$ by $S_{\text {reg }}$ and $\mathfrak{t} . S_{\text {reg }} \subset D_{\text {reg }}$ acts on $S_{\text {reg }} \otimes$ Sym $\mathfrak{t} \otimes \mathbb{C}[\hbar]$ by multiplication on the left factor, and $(\operatorname{Sym} \mathfrak{t})^{W}$ acts by multiplication on the right factor. Following [BMO11], we will describe the action of $x \in \mathfrak{t}$ in terms of the degenerate Hecke algebra $\mathcal{H}_{\hbar}$. This is the algebra generated by $\mathbb{C}[W]$ and $\operatorname{Sym} \mathfrak{t} \otimes \mathbb{C}[\hbar]$, subject to the relations

$$
s_{\alpha} x-s_{\alpha}(x) s_{\alpha}=-\hbar\langle\alpha, x\rangle
$$

where $s_{\alpha}$ is the reflection associated with the simple root $\alpha$ and $x \in \mathfrak{t}$. Here our $\hbar$ corresponds to the variable $-t$ in [BMO11].

There is a natural identification of Sym $\mathfrak{t} \otimes \mathbb{C}[\hbar]$ with the $\mathcal{H}_{\hbar}$-module $J:=\mathcal{H}_{\hbar} \otimes_{\mathbb{C}[W]} \mathbb{C}$, where $\mathbb{C}[W]$ acts on $\mathbb{C}$ via the trivial representation, inducing an identification of the righthand side of Equation 14 with $S_{\text {reg }} \otimes J$. By our definition of $Q_{\text {reg }}$, the element $x \in \mathfrak{t}$ acts via the covariant derivative $\hbar \partial_{x}-\widetilde{\psi}(x) \star$. By [BMO11, Theorem 3.2], the latter acts on $\left(Q_{\mathrm{reg}}^{!}\right)^{W} \cong S_{\mathrm{reg}} \otimes J$ via the operator

$$
\nabla_{x}^{B M O}:=\hbar \partial_{x}-(x+\hbar\langle\rho, x\rangle)+\hbar \sum_{\alpha \in \Delta_{+}}\langle\alpha, x\rangle \frac{q^{\alpha}}{1-q^{\alpha}}\left(s_{\alpha}-1\right),
$$

where $\partial_{x}$ acts on the left factor and $x$ and $s_{\alpha}$ are viewed as elements of $\mathcal{H}_{\hbar}$, which acts on the right factor.

The $S_{\text {reg-module }}\left(Q_{\text {reg }}^{!}\right)^{W}$ has infinite rank. In order to apply certain results on holonomic D-modules, we will need to base-change to $T_{\text {reg }} \subset$ Spec $S_{\text {reg }}$ and consider various finite rank quotients, obtained by specialising equivariant parameters, or equivalently central characters of the Hecke algebra. The center of $\mathcal{H}_{\hbar}$ is equal to $(\operatorname{Sym} \mathfrak{t})^{W} \otimes \mathbb{C}[\hbar]$. Thus, given any $c \in \mathfrak{t}^{*} / W$ and $t \in \mathbb{C}$, we can consider the corresponding evaluation module $\mathbb{C}_{c}$ of $(\operatorname{Sym} \mathfrak{t})^{W}$, and define the $\mathcal{H}_{\hbar}$-module

$$
J^{c, t}:=\operatorname{Sym} \mathfrak{t} \otimes_{(\operatorname{Sym} \mathfrak{t})^{W}} \mathbb{C}_{c} \otimes_{\mathbb{C}[\hbar]} \mathbb{C}[\hbar] /(\hbar+t)
$$


and the $D_{\hbar}\left(T_{\text {reg }}\right)$-module

$$
Q^{c, t}:=\left(Q_{T_{\text {reg }}}^{!}\right)^{W} \otimes_{(\operatorname{Sym} \mathfrak{t})^{W}} \mathbb{C}_{c} \otimes_{\mathbb{C}[\hbar]} \mathbb{C}[\hbar] /(\hbar+t)
$$

As a vector space, we have an isomorphism $Q^{c, t} \cong \mathcal{O}\left(T_{\text {reg }}\right) \otimes J^{c, t}$. Note that $J^{c, t}$ has complex dimension $|W|$.

Definition 7.12. Che05, Definition 1.1.39] The affine Knizhnik-Zamolodchikov (AKZ) connection is the following $\mathcal{H}_{\hbar}$-valued connection on $T_{\text {reg }}$ :

$$
\nabla_{x}^{A K Z}:=\partial_{x}-x+\hbar \sum_{\alpha \in \Delta_{+}}\langle\alpha, x\rangle \frac{s_{\alpha}}{q^{\alpha}-1}
$$

Let $\mathcal{M}:=\mathcal{O}\left(T_{\text {reg }}\right) \otimes J$ be the $D\left(T_{\text {reg }}\right)$-module on which $\iota(x)$ acts via $\nabla_{x}^{A K Z}$. As above, we write $\mathcal{M}^{c, t}$ for the corresponding specialisations at $(c, t) \in \mathfrak{t}^{*} / W \times \mathbb{C}$.

Let $\pi$ be the $\mathbb{C}$-linear involution of $\mathcal{O}\left(T_{\text {reg }}\right) \otimes J$ defined by

$$
\pi(f(q) \otimes a)=f\left(q^{-1}\right) \otimes a
$$

Note that $\pi \circ \partial_{x} \circ \pi=-\partial_{x}$.

Lemma 7.13. The isomorphism $Q^{c, 1} \cong \mathcal{O}\left(T_{\mathrm{reg}}\right) \otimes J^{c, 1}$ identifies the action of $\delta^{-1} \nabla_{x_{\rho}}^{B M O} \delta$ with the action of $\pi \circ \nabla_{x}^{A K Z} \circ \pi$ specialised at $\hbar=-1$.

Proof. In the ring $E_{\text {reg, }}^{!}$, we have the equality

$$
\delta^{-1} \tilde{\psi}\left(x_{\rho}\right) \delta=\tilde{\psi}\left(x_{\rho}\right)+\hbar \sum_{\alpha \in \Delta^{+}}\langle\alpha, x\rangle \frac{q^{\alpha}}{q^{\alpha}-1} .
$$

The action of this element on $\left(Q_{\text {reg }}^{!}\right)^{W}$ is given, via 15 , by

$$
\begin{aligned}
& \hbar \partial_{x}-x+\hbar \sum_{\alpha \in \Delta_{+}}\langle\alpha, x\rangle \frac{q^{\alpha}}{1-q^{\alpha}}\left(s_{\alpha}-1\right)+\hbar \sum_{\alpha \in \Delta^{+}}\langle\alpha, x\rangle \frac{q^{\alpha}}{1-q^{\alpha}} \\
= & \hbar \partial_{x}-x+\hbar \sum_{\alpha \in \Delta_{+}}\langle\alpha, x\rangle \frac{q^{\alpha}}{1-q^{\alpha}} s_{\alpha} \\
= & \hbar \partial_{x}-x+\hbar \sum_{\alpha \in \Delta_{+}}\langle\alpha, x\rangle \frac{s_{\alpha}}{q^{-\alpha}-1} .
\end{aligned}
$$

Conjugating by $\pi$ gives

$$
-\hbar \partial_{x}-x+\hbar \sum_{\alpha \in \Delta_{+}}\langle\alpha, x\rangle \frac{s_{\alpha}}{q^{\alpha}-1}
$$


and setting $\hbar=-1$ yields

$$
\partial_{x}-x-\sum_{\alpha \in \Delta_{+}}\langle\alpha, x\rangle \frac{s_{\alpha}}{q^{\alpha}-1} .
$$

Via Lemma 7.13, we will derive the differential relations of Theorem 7.11 from known relations in $\mathcal{M}^{c, t}$.

The key tool is the identification, due to Cherednik and Matsuo, of $\mathcal{M}^{c, t}$ with a certain scalar $D\left(T_{\text {reg }}\right)$-module which Cherednik calls the quantum many body problem, and which BMO11] refer to as the trigonometric Calogero-Moser system (see Opd00 for an introduction). It may be described as follows. Fix a fixed Weyl invariant non-degenerate quadratic form on $\mathfrak{t}$. The Calogero-Moser module $\mathcal{C} \mathcal{M}^{t}$ is a module over the ring $D\left(T_{\text {reg }}\right) \otimes(\operatorname{Sym} \mathfrak{t})^{W}$. As a module over $D\left(T_{\text {reg }}\right)$, it is simply $D\left(T_{\text {reg }}\right)$ itself. The action of $a \in(\operatorname{Sym} \mathfrak{t})^{W}$ is given by right-multiplication by $\eta_{t}(a) \in D_{\text {reg }}$, where $\eta_{t}:(\operatorname{Sym} \mathfrak{t})^{W} \rightarrow D_{\text {reg }}$ is the unique algebra homomorphism satisfying the following two properties.

1. $\eta_{t}(a)$ lies in the algebra generated by $\iota(b), b \in \operatorname{Sym} \mathfrak{t}$ and $\frac{1}{1-q^{\alpha}}, \alpha \in \Delta_{+}$, and has an expansion

$$
\eta_{t}(a)=\iota(a)+\sum_{\alpha \in \mathbb{Z}^{>0} \Delta_{+}} q^{\alpha} p_{\alpha}(a)
$$

where the $p_{\alpha}(a)$ are constant coefficient differential operators.

2. Let $C$ be the element of $\left(\mathrm{Sym}^{2} \mathfrak{t}\right)^{W}$ determined by our fixed quadratic form. Let $L \in D\left(T_{\text {reg }}\right)$ be the associated constant coefficient differential operator, sometimes called the Laplacian.

$$
\eta_{t}(C)=\iota(C)-t(t-1) \sum_{\alpha \in \Delta_{+}} \frac{(\alpha, \alpha)}{\left(q^{\alpha / 2}-q^{-\alpha / 2}\right)^{2}}
$$

The relation $\left[\eta_{t}(a), \eta_{t}(C)\right]=0$ imposes a recursive relation on the coefficients $p_{\alpha}(a)$, which fixes them uniquely.

Lemma 7.14. $\eta_{1}(a)=\iota(a)$.

Proof. The right-hand side defines a homomorphism which clearly satisfies both conditions above.

As above, given $c \in \mathfrak{t} / W$, we define $\mathcal{C M}^{c, t}:=\mathcal{C M}^{t} \otimes_{(\operatorname{Sym} t)}{ }^{W} \mathbb{C}_{c}$. This specialisation imposes the relations

$$
\eta_{t}(a) \cdot 1=a(c)
$$


for all $a \in(\operatorname{Sym} \mathfrak{t})^{W}$.

Consider the inclusion $1 \rightarrow(\operatorname{Sym} \mathfrak{t})^{W}$. This extends to a $D\left(T_{\text {reg }}\right)$-linear map $\Psi^{t}$ : $D\left(T_{\text {reg }}\right) \rightarrow \mathcal{M}^{t}$, taking 1 to $1 \otimes 1$.

Theorem 7.15. The map $\Psi^{t}$ induces a homomorphism of $D\left(T_{\mathrm{reg}}\right)$-modules

$$
\Psi^{c, t}: \mathcal{C M}^{c, t} \rightarrow \mathcal{M}^{c, t}
$$

Proof. The proposition is essentially [Che05, Theorem 1.2.11] (see also [Mat92]). Since both references state their results in terms of sheaves of solutions rather than D-modules, we make the translation here. To do so, we now switch from algebraic D-modules to D-modules on the analytic space associated to $T_{\text {reg. }}$. We write $\mathcal{D}_{\text {an }}$ for the sheaf of differential operators on this space and $\mathcal{O}_{\text {an }}$ for the sheaf of analytic functions. Similarly, $\mathcal{M}^{c, t}$ and $\mathcal{C} \mathcal{M}^{c, t}$ have analytic versions denoted $\mathcal{M}_{\text {an }}^{c, t}$ and $\mathcal{C M}_{\text {an }}^{c, t}$.

The homomorphism $\Psi^{t}$ induces a map of sheaves

$$
\Psi_{\text {an }}^{t, c}: \mathscr{H}_{0 m_{\mathcal{D}_{\text {an }}}}\left(\mathcal{M}_{\text {an }}^{c, t}, \mathcal{O}_{\text {an }}\right) \rightarrow \mathscr{H}_{o m_{\mathcal{D}_{\text {an }}}}\left(\mathcal{D}_{\text {an }}, \mathcal{O}_{\text {an }}\right),
$$

which is given by the formula

$$
\sigma \mapsto(d \mapsto \sigma(d \cdot 1))
$$

for $d$ a section of $\mathcal{D}_{\text {an }}$ and $\sigma$ a section of $\mathscr{H}_{o m_{\mathcal{D}_{\text {an }}}}\left(\mathcal{M}_{\text {an }}^{c, t}, \mathcal{O}_{\text {an }}\right)$. By [Che05, Theorem 1.2.12], this map factors through $\mathscr{H}_{o m_{\mathcal{D}_{\text {an }}}}\left(\mathcal{C M}_{\text {an }}^{c, t}, \mathcal{O}_{\text {an }}\right)$, which implies that the map $\mathcal{D}_{\text {an }} \rightarrow \mathcal{M}_{\text {an }}^{c, t}$ factors through the projection $\mathcal{D}_{\text {an }} \rightarrow \mathcal{C} \mathcal{M}_{\text {an }}^{c, t}$. Taking global sections, we find that $\Psi_{\text {an }}^{t, c}$ factors through the projection from $D_{\text {an }}\left(T_{\text {reg }}\right)$ to $\mathcal{C} \mathcal{M}_{\text {an }}^{c, t}$. Since the algebraic modules sit inside of the analytic ones, this implies the statement that we need.

It follows from Theorem 7.15 that for any $a \in(\operatorname{Sym} \mathfrak{t})^{W}$, we have the equality

$$
\eta_{t}(a) \cdot 1 \otimes 1=1 \otimes a
$$

in $\mathcal{M}$. Applying $\pi$ to both sides, we get

$$
\pi \circ \eta_{t}(a) \cdot 1 \otimes 1=\pi \cdot 1 \otimes a .
$$

We can rewrite the left-hand side as $\pi \circ \eta_{t}(a) \circ \pi \cdot 1 \otimes 1$. Specializing at $(c, 1)$ and recalling $\eta_{1}(a)=\iota(a)$, we obtain the equality

$$
\pi \circ \iota(a) \circ \pi \cdot 1 \otimes 1=1 \otimes a(c)
$$

in $\mathcal{M}^{c, 1}$. By Lemma 7.13, we can identify $\pi \circ \iota(a) \circ \pi$ with the action of $\delta^{-1} \iota\left(a_{\rho}\right) \delta$ on $Q^{c, 1}$. 
We thus obtain our key result.

Corollary 7.16. For any $a \in(\operatorname{Sym} \mathfrak{t})^{W}$, the following holds in $Q^{c, 1}$ :

$$
\delta^{-1} \iota\left(a_{\rho}\right) \delta \cdot 1 \otimes 1=1 \otimes a(c)
$$

Proof of Theorem 17.11; Consider the induced map $\bar{\Psi}: D_{\text {reg }} / \hbar D_{\text {reg }} \rightarrow\left(Q_{\text {reg }}^{!}\right)^{W} / \hbar\left(Q_{\text {reg }}^{!}\right)^{W}$. We have $D_{\text {reg }} / \hbar D_{\text {reg }} \cong S_{\text {reg }} \otimes \operatorname{Sym} \mathfrak{t} \cong\left(Q_{\text {reg }}^{!}\right)^{W} / \hbar\left(Q_{\text {reg }}^{!}\right)^{W}$. Under these identifications, Equa-

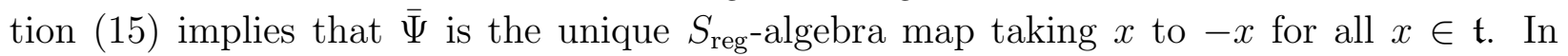
particular, $\bar{\Psi}$ is an isomorphism. Since $D_{\text {reg }}$ and $\left(Q_{\text {reg }}^{!}\right)^{W}$ are graded modules over $\mathbb{C}[\hbar]$ with bounded below grading and $\left(Q_{\text {reg }}^{!}\right)^{W}$ is torsion-free as a module over $\mathbb{C}[\hbar]$, we conclude (by a standard argument) that $\Psi$ is also an isomorphism.

Next, we show that for any $a \in(\operatorname{Sym} \mathfrak{t})^{W}$, we have $\delta^{-1} \iota\left(a_{\rho}\right) \delta \cdot(1 \otimes 1)=1 \otimes a$. It is enough to show that this equality holds after localizing to $T_{\text {reg }}$ and specializing $\left(Q_{\text {reg }}^{\text {! }}\right)^{W}$ to a generic point in Spec $(\operatorname{Sym} \mathfrak{t})^{W} \otimes \mathbb{C}[\hbar]$. By homogeneity, we may assume $\hbar=-1$, i.e. $t=1$ in the notation of this section. The desired equality is then Equation 18 .

The following result now follows immediately from Theorem 7.8, Proposition 7.10 and Theorem 7.11,

Theorem 7.17. We have an isomorphism of $D_{\mathrm{reg}} \otimes(\operatorname{Sym} \mathfrak{t})^{W}$-modules $M_{\mathrm{reg}}^{W} \cong\left(Q_{\mathrm{reg}}^{!}\right)^{W}$, taking $1 \otimes 1$ to $1 \otimes 1$. In particular, Conjectures 5.1 and 5.5 hold for the Springer resolution. Proof. The first statement follows from Theorem 7.8, Proposition 7.10 and Theorem 7.11 . This establishes that Conjecture 5.5 holds for the Springer resolution, and Conjecture 5.1 follows from the discussion in Section 5.3 .

\section{A A geometric description of highest weights an appendix by Ben Webster}

As in the main text, let $\tilde{X} \rightarrow X$ be a conical symplectic resolution, and $T$ an algebraic torus acting Hamiltonianly on $\tilde{X}$. As before, the algebra $\mathscr{A}$ is the universal deformation quantization of $X$; it will be more convenient for us to instead work with a specialization of this, though. Based on the work of Bezrukavnikov and Kaledin, we have a sheaf $\mathscr{D}^{c}$ on $\tilde{X}$ quantizing the structure sheaf for each choice of $c \in H^{2}(\tilde{X} ; \mathbb{C})$. The global sections of this sheaf are $\mathscr{A}^{c}$, the specialization of $\mathscr{A}$ at $c$.

Recall the quantization exact sequence (2)

$$
0 \rightarrow H_{2}(\tilde{X} ; \mathbb{C}) \oplus \mathbb{C} \hbar \rightarrow \mathscr{A}_{0}^{2} \rightarrow \mathfrak{t} \rightarrow 0 .
$$


As discussed in Remark 2.2, a splitting of this exact sequence is a quantized comoment map $\mathfrak{t} \rightarrow \mathscr{A}_{0}^{2}$. Any two such maps differ by an element of $\operatorname{Hom}\left(\mathfrak{t}, H_{2}(\tilde{X}) \oplus \mathbb{C} \hbar\right)$.

By universality, we can choose a $T$-equivariant isomorphism a: $\mathscr{A} \cong \mathscr{A}^{o p}$ sending $\hbar \mapsto-\hbar$. For any quantized comoment map $\mathrm{m}$, we have that $\mathrm{a}(\mathrm{m})$ is again a quantum comoment map, and this is an anti-automorphism of affine spaces. The fixed points form a torsor over $\operatorname{Hom}\left(\mathfrak{t}, H_{2}(\tilde{X})\right)$; all the corresponding comoment maps agree after taking the quotient at $c=0$.

We will be interested in how this comoment map interacts with the B-algebra mentioned earlier. This algebra depends on $\xi \in \mathfrak{t}$ and is given by

$$
B(\mathscr{A}):=\mathscr{A}_{0} / \sum_{\langle\mu, \xi\rangle>0}\left\{a b \mid a \in \mathscr{A}_{\mu}, b \in \mathscr{A}_{-\mu}\right\} .
$$

This algebra naturally acts on the weight spaces of any $\mathscr{A}$-module which minimize $\langle\mu, \xi\rangle$. Thus, the induced map $\mathfrak{t} \rightarrow B(\mathscr{A})$ also controls how $\mathfrak{t}$ acts on the lowest weight spaces of the different simple modules in category $\mathcal{O}$.

We use the construction of standard modules in category $\mathcal{O}$ given in [BLPW16, §5.1]. This is done by analyzing the structure of $\mathscr{D}^{c}$ near any fixed point $x$. By the Darboux theorem, a formal neighborhood of $x$ in $\tilde{X}$ is $T$-equivariantly symplectomorphic to the tangent space, and thus the completion of $\mathscr{D}^{c}$ here to a completed Weyl algebra (with parameter $\hbar$ ). Passing to $T$ - and $\mathbb{G}_{m}$-locally finite vectors gives a copy of the Weyl algebra $A_{x}^{c}$, as shown in BLPW16, Lem. 5.2]. Since we only pass to $\mathbb{G}_{m}$-locally finite vectors rather than invariants as in [BLPW16, Lem. 5.2], we obtain a family over $\mathbb{C}[\hbar]$, which we identify with polynomials on $T_{x} \tilde{X}$ with the Moyal star product. The algebra $A_{x}^{c}$ has its own $B$-algebra $B_{x}^{c} \cong \mathbb{C}[\hbar]$. Restriction induces a map $\mathscr{A} \rightarrow A_{x}^{c}$ which in turn induces a map $B(\mathscr{A}) \rightarrow B_{x}^{c}$. The induced map $\mathscr{A}_{0}^{2} \rightarrow \mathbb{C} \hbar \subset B_{x}^{c}$ is exactly the map $w_{x}^{c}$ defined in Section 3.7.

Definition A.1. For each fixed point $x$, let $\mathrm{m}_{x}: \mathfrak{t} \rightarrow \mathscr{A}_{0}^{2}$ be the unique comoment map whose image in $B_{x}^{c}$ is 0 for all $c$.

This comoment map is easily constructed by considering an arbitrary moment map, and noting that this induces a linear map $\mathfrak{t} \rightarrow H_{2}(\tilde{X}) \oplus \mathbb{C} \hbar$ by considering the values in $B_{x}^{c}$ for different $c$; thus subtracting this, we arrive at $\mathrm{m}_{x}$.

Of course, this splitting has a dual splitting $\mathscr{A}_{0}^{2} \rightarrow H_{2}(\tilde{X}) \oplus \mathbb{C} \hbar$, which is exactly the map $w_{x}^{c}$, thinking of $c$ as an element of the dual space of $H_{2}(\tilde{X}) \oplus \mathbb{C} \hbar$. The differences $\mathbf{m}_{x}-\mathrm{m}_{y}$ and $w_{x}^{c}-w_{y}^{c}$ both define maps $\mathfrak{t} \rightarrow H_{2}(\tilde{X}) \oplus \mathbb{C} \hbar$, so we can compare them. By the usual properties of dual splittings, we have that:

Lemma A.2. We have an equality $\mathrm{m}_{x}-\mathrm{m}_{y}=w_{y}^{c}-w_{x}^{c}$. 
For each fixed point $x$, there is a natural decomposition of $T_{x} \tilde{X}=T_{x}^{+} \tilde{X} \oplus T_{x}^{-} \tilde{X}$ according to whether the weight of $\xi$ is positive or negative. Let $\left\{\chi_{i}\right\}$ denote the $T$-weights of $T_{x}^{+} \tilde{X}$ (with multiplicity); of course, since $\xi$ is symplectic, the weights on $T_{x}^{-} \tilde{X}$ are just $\left\{-\chi_{i}\right\}$. We define an element $\chi_{x} \in \mathfrak{t}^{*}$ by $\chi_{x}=1 / 2 \sum \chi_{i}$. More canonically, we can think of this as the weight of $T$ on $\operatorname{det}\left(T_{x}^{+} \tilde{X}\right)^{1 / 2}$.

We have an exact sequence

$$
0 \rightarrow H_{2}(\tilde{X}) \rightarrow H_{2}^{T}(\tilde{X}) \rightarrow \mathfrak{t} \rightarrow 0
$$

This is the dual to the cohomology exact sequence (6), except that we have removed the $\mathbb{G}_{m}$-equivariance.

For each $x$, we have a splitting $\iota_{x}: \mathfrak{t} \rightarrow H_{2}^{T}(\tilde{X})$ given by push-forward from the fixed point. We define $\rho_{x, y}=\iota_{x}-\iota_{y}: \mathfrak{t} \rightarrow H_{2}(\tilde{X})$; as usual, the difference of two splittings is a map to the kernel of the map which is split. This map also has a dual $\rho_{x, y}^{*}: H^{2}(\tilde{X}) \rightarrow \mathfrak{t}^{*}$.

Theorem A.3. For all fixed points $x, y$, we have

$$
\mathrm{m}_{x}-\mathrm{m}_{y}=\rho_{x, y}-\left(\chi_{x}-\chi_{y}\right) \hbar \in \operatorname{Hom}\left(\mathfrak{t}, H_{2}(\tilde{X}) \oplus \mathbb{C} \hbar\right) .
$$

That is, for any noncommutative comoment map $\mathrm{m}$, and any $u \in \mathfrak{t}$, the difference between the action of $\mathrm{m}(u)$ on $B_{y}^{c}$ and on $B_{x}^{c}$ is

$$
\left(w_{y}^{c}-w_{x}^{c}\right)(u)=\left\langle u, \rho_{x, y}^{*}(c)-\left(\chi_{x}-\chi_{y}\right)\right\rangle \hbar .
$$

Corollary A.4. The composition $U(\mathfrak{t}) \rightarrow B \rightarrow \oplus B_{x}^{c}$ is surjective if and only if $\rho_{x, y}^{*}(c)-$ $\left(\chi_{x}-\chi_{y}\right)$ is not zero for all $x, y$. In particular, if $\rho_{x, y} \neq 0$ for all $x, y$, the map $B \rightarrow \oplus_{x} B_{x}^{c}$ is surjective for c away from finitely many proper subspaces.

Proof. We must have $\mathrm{m}_{x}-\mathrm{m}_{y}=\rho_{x, y}^{\prime}-k_{x, y} \hbar$ for some $\rho_{x, y}^{\prime} \in \operatorname{Hom}\left(\mathfrak{t}, H^{2}(\tilde{X})\right)$ and $k_{x, y} \in \mathfrak{t}^{*}$.

First, we note that $k_{x, y}=k_{x}-k_{y}$ where $k_{x}=\frac{1}{2}\left(\mathrm{a}\left(\mathrm{m}_{x}\right)-\mathrm{m}_{x}\right)$. As in the proof of [BLPW16, Lem. 5.2], we choose Weyl generators $\left\{x_{i}, y_{i}\right\}$ such that all $x_{i}$ have positive weight for $\xi$ and weight $\chi_{i}$ for $T$; the $y_{i}$ thus have weight $-\chi_{i}$. In these coordinates, we have $\mathrm{m}_{x}(t)=\sum\left\langle t, \chi_{i}\right\rangle x_{i} y_{i}$. We have a weight preserving anti-automorphism on $A_{f i x}^{0}$ given by $x_{i} \mapsto x_{i}, y_{i} \mapsto y_{i}, \hbar \mapsto-\hbar$, and the image of $\mathrm{m}_{x}(t)$ under this map is

$$
\sum\left\langle t, \chi_{i}\right\rangle y_{i} x_{i}=\sum\left\langle t, \chi_{i}\right\rangle x_{i} y_{i}-\hbar\left\langle t, \chi_{i}\right\rangle \text {. }
$$

Thus, we have that $k_{x}=\hbar / 2 \sum \chi_{i}=\chi_{x}$, and the second component of the formula (19) is correct.

We can calculate $\rho_{x, y}^{\prime}$ by considering the effect of tensor product with quantized line 
bundles. Whenever we have two classes $c, c^{\prime}$ with $c-c^{\prime} \in H^{2}(\tilde{X}, \mathbb{Z})$, we can quantize the unique line bundle with this Chern class to a $\mathscr{D}^{c}-\mathscr{D}^{c}$-bimodule. The action

$$
t \cdot s=\mathrm{m}_{x}(t) s-s \mathrm{~m}_{x}(t)
$$

induces an action of $\mathfrak{t}$ on the sections of this quantization and thus a $T$-equivariant structure on the corresponding line bundle. This is uniquely characterized by the fact that this action is trivial on the fiber over $x$. Thus, its weight on the fiber over $y$ is exactly $\rho_{y, x}^{*}\left(c-c^{\prime}\right)$, and we can conclude that

$$
\left\langle\rho_{x, y}^{\prime}(t), c-c^{\prime}\right\rangle=\left\langle t, \rho_{x, y}^{*}\left(c-c^{\prime}\right)\right\rangle .
$$

Since this equation holds for all $c-c^{\prime} \in H^{2}(\tilde{X}, \mathbb{Z})$, we must have that $\rho_{x, y}^{\prime}=\rho_{x, y}$ and (19) holds.

Example A.5. If $\tilde{X}=T^{*} G / B$, then let $\mathfrak{n}_{-} \subset \mathfrak{g}$ be the nilpotent subalgebra given by the negative $\xi$-weight spaces of $\mathfrak{g}$ and $\mathfrak{t}$ be the Cartan subalgebra given by its invariants. At each $T$-fixed points, we have that $T^{*} \tilde{X} \cong \mathfrak{g} / \mathfrak{t}$. Thus at each fixed point, we have that $T_{x}^{-} \tilde{X} \cong \mathfrak{n}_{-}$ as a $T$-representation, and $\chi_{x}=-\rho$ for all $x$.

Recall that after identifying $H_{2}(G / B ; \mathbb{C}) \cong \mathfrak{t}$, the maps $\rho_{x, y}: \mathfrak{t} \rightarrow \mathfrak{t}$ are simply $w_{x}-w_{y}$ for the corresponding elements of the Weyl group. In particular, this shows that the $B$-algebra will surject to the sum $B_{x}^{c}$ (and thus we will have the full expected number of Verma modules) if and only if $w c-w^{\prime} c \neq 0$ for all $w, w^{\prime} \in W$. That is, if $c$ is regular.

Readers familiar with the theory of category $\mathcal{O}$ might be confused by the absence of " $\rho$ shifts" but we have already dealt with these in choosing our conventions for $\mathscr{D}^{c}$ (these coincide with the conventions of [BB81]); for example, the usual microlocal differential operators are $\mathscr{D}^{\rho}$, so this is consistent with the fact that principal block is regular.

\section{References}

[AFO18] Mina Aganagic, Edward Frenkel, and Andrei Okounkov, Quantum q-langlands correspondence, Transactions of the Moscow Mathematical Society 79 (2018), 1-83.

[BB81] Alexandre Beĭlinson and Joseph Bernstein, Localisation de g-modules, C. R. Acad. Sci. Paris Sér. I Math. 292 (1981), no. 1, 15-18.

[BD00] Roger Bielawski and Andrew S. Dancer, The geometry and topology of toric hyperkähler manifolds, Comm. Anal. Geom. 8 (2000), no. 4, 727-760. 
[BFN18] Alexander Braverman, Michael Finkelberg, and Hiraku Nakajima, Towards a mathematical definition of Coulomb branches of 3-dimensional $\mathcal{N}=4$ gauge theories, II, Adv. Theor. Math. Phys. 22 (2018), no. 5, 1071-1147.

[BGS96] Alexander Beilinson, Victor Ginzburg, and Wolfgang Soergel, Koszul duality patterns in representation theory, J. Amer. Math. Soc. 9 (1996), no. 2, 473527.

[BLPW12] Tom Braden, Anthony Licata, Nicholas Proudfoot, and Ben Webster, Hypertoric category $\mathcal{O}$, Adv. Math. 231 (2012), no. 3-4, 1487-1545.

[BLPW16] Tom Braden, Anthony Licata, Nicholas Proudfoot, and Ben Webster, Quantizations of conical symplectic resolutions II: category $\mathcal{O}$ and symplectic duality, Astérisque (2016), no. 384, 75-179, with an appendix by I. Losev.

[BMO11] Alexander Braverman, Davesh Maulik, and Andrei Okounkov, Quantum cohomology of the Springer resolution, Adv. Math. 227 (2011), no. 1, 421-458.

[BPW16] Tom Braden, Nicholas Proudfoot, and Ben Webster, Quantizations of conical symplectic resolutions I: local and global structure, Astérisque (2016), no. 384, $1-73$.

[Che05] Ivan Cherednik, Double affine Hecke algebras, London Mathematical Society Lecture Note Series, vol. 319, Cambridge University Press, Cambridge, 2005.

[ES] Pavel Etingof and Douglas Stryker, Short star-products for filtered quantizations, $i$, arXiv:1909.13588.

[Hik17] Tatsuyuki Hikita, An Algebro-Geometric Realization of the Cohomology Ring of Hilbert Scheme of Points in the Affine Plane, Int. Math. Res. Not. IMRN (2017), no. 8, 2538-2561.

[Hil16] Justin Hilburn, Hypergeometric systems and projective modules in hypertoric category $\mathcal{O}, 2016$, Thesis (Ph.D.)-University of Oregon.

[Hum78] James E. Humphreys, Introduction to Lie algebras and representation theory, Graduate Texts in Mathematics, vol. 9, Springer-Verlag, New York-Berlin, 1978, Second printing, revised.

[Kon99] Hiroshi Konno, Equivariant cohomology rings of toric hyperkähler manifolds, Quaternionic structures in mathematics and physics (Rome, 1999), Univ. Studi Roma "La Sapienza", Rome, 1999, pp. 231-240. 
$\left[\mathrm{KTW}^{+} 19 \mathrm{a}\right]$ Joel Kamnitzer, Peter Tingley, Ben Webster, Alex Weekes, and Oded Yacobi, Highest weights for truncated shifted Yangians and product monomial crystals, J. Comb. Algebra 3 (2019), no. 3, 237-303.

$\left[\mathrm{KTW}^{+} 19 \mathrm{~b}\right]$ - On category $\mathcal{O}$ for affine Grassmannian slices and categorified tensor products, Proc. Lond. Math. Soc. (3) 119 (2019), no. 5, 1179-1233.

[Los] Ivan Losev, Deformations of symplectic singularities and Orbit method for semisimple Lie algebras, arXiv:1605.00592.

[Mat92] Atsushi Matsuo, Integrable connections related to zonal spherical functions, Inventiones mathematicae 110 (1992), no. 1, 95-121.

[MO19] Davesh Maulik and Andrei Okounkov, Quantum groups and quantum cohomology, Astérisque (2019), no. 408, ix+209.

[MP15] Michael McBreen and Nicholas Proudfoot, Intersection cohomology and quantum cohomology of conical symplectic resolutions, Algebr. Geom. 2 (2015), no. 5, 623-641.

[MS13] Michael B. McBreen and Daniel K. Shenfeld, Quantum cohomology of hypertoric varieties, Lett. Math. Phys. 103 (2013), no. 11, 1273-1291.

[Nam10] Yoshinori Namikawa, Poisson deformations of affine symplectic varieties, II, Kyoto J. Math. 50 (2010), no. 4, 727-752.

[Oko15] Andrei Okounkov, Enumerative geometry and geometric representation theory, Algebraic Geometry: Salt Lake City (2015), 419-457.

[OP10] Andrei Okounkov and Rahul Pandharipande, The quantum differential equation of the hilbert scheme of points in the plane, Transformation Groups 15 (2010), no. 4, 965-982.

[Opd00] Eric M. Opdam, Lecture notes on Dunkl operators for real and complex reflection groups, MSJ Memoirs, vol. 8, Mathematical Society of Japan, Tokyo, 2000, With a preface by Toshio Oshima.

[OS] Andrei Okounkov and Andrey Smirnov, Quantum difference equation for Nakajima varieties, arXiv:1602.09007.

[Pro14] Nicholas Proudfoot, Hypertoric Poisson homology in degree zero, Alg. Geom. 2 (2014), 1-10. 
[Web] Ben Webster, Koszul duality between higgs and coulomb categories $\mathcal{O}$, arXiv:1611.06541.

[Web17] Ben Webster, On generalized category $\mathcal{O}$ for a quiver variety, Math. Ann. 368 (2017), no. 1-2, 483-536. 